\title{
Electrocutaneous stimulation to close the loop in myoelectric prosthesis control
}

\author{
Dissertation \\ zur Erlangung des mathematisch-naturwissenschaftlichen \\ Doktorgrades \\ Doctor rerum naturalium \\ der Georg-August-Universität Göttingen \\ im Promotionsprogramm \\ Theoretical and Computational Neuroscience \\ der Georg-August University School of Science (GAUSS)
}

vorgelegt von

Cornelia Hartmann

aus Essen

Göttingen 2016 


\section{Betreuungsausschuss}

Prof. Dr. Dr. Dario Farina, Institute of Neurorehabilitation Systems, Universitätsmedizin Göttingen

Prof. Dr. Florentin Wörgötter, III. Physikalisches Institut - Biophysik, Georg-August-Universität Göttingen

Prof. Dr. Marc Timme, Abt. Netzwerk Dynamik,

Max-Planck-Institut für Dynamik und Selbstorganisation, Göttingen

Dr. Bernhard Graimann, Translational Research and Knowledgemanagement, Otto Bock HealthCare GmbH, Duderstadt

\section{Referent}

Prof. Dr. Dr. Dario Farina, Institute of Neurorehabilitation Systems, Universitätsmedizin Göttingen

\section{Koreferent}

Prof. Dr. Florentin Wörgötter, III. Physikalisches Institut - Biophysik, Georg-August-Universität Göttingen

\section{Weitere Mitglieder der Prüfungskommission}

Prof. Dr. Jens Frahm, Biomedizinische NMR Forschungs GmbH, Max-Planck-Institut für biophysikalische Chemie

Prof. Dr. Stefan Luther, Biomedizinische Physik, Max-Planck-Institut für Dynamik und Selbstorganisation

Prof. Dr. Marc Timme, Abt. Netzwerk Dynamik, Max-Planck-Institut für Dynamik und Selbstorganisation, Göttingen

Dr. Bernhard Graimann, Translational Research and Knowledgemanagement, Otto Bock HealthCare GmbH, Duderstadt

Tag der mündlichen Prüfung: 22. November 2016 




\section{Acknowledgements}

I would like to express my sincere gratitude to Prof. Dario Farina for his excellent guidance and his endorsement throughout all stages of my doctoral project. His uplifting nature and positive attitude keep encouraging me to work hard, and pursue my goals.

Furthermore, I would like to thank the members of my Thesis Committee for their continued support of my work throughout the years, and their excellent advice on diverse aspects of my work.

I am also much obliged to Dr. Strahinja Došen for his continued scientific guidance during my doctoral project, but also for his patience. Last but not least, I would like to express my deepest gratitude to my husband Matthias - loving father of our little sunshine - and my parents for their care and continuous encouragement. Without them, the completion of this thesis would not have happened.

The research leading to the results presented in this dissertation has received funding from the People Programme (Marie Curie Actions) of the European Union's Seventh Framework Programme FP7/20072013/ under REA grant agreement no. 286208 (MYOSENS). 



\begin{abstract}
Current commercially available prosthetic systems still lack sensory feedback and amputees are forced to maintain eye-contact with the prosthesis when interacting with their environment. Electrocutaneous stimulation is a promising approach to convey sensory feedback via the skin. However, when discussed in the context of prosthetic applications, it is often refused due to its supposed incompatibility with myocontrol.

This dissertation now addresses electrocutaneous stimulation as means to provide sensory feedback to prosthesis users, and its implications on myoelectric control, possible use for improved or accelerated mastering of prosthesis control through closing of the control loop, as well as its potential in aiding in the embodiment of prosthetic components. First, a comparison of different paradigms for encoding sensory feedback variables in electrocutaneous stimulation patterns was done. For this, subject ability to employ spatially and intensity-coded electrocutaneous feedback in a simulated closed-loop control task was evaluated. The task was to stabilise an invisible virtual inverted pendulum under ideal feedforward control conditions (joystick). Pendulum inclination was either presented spatially (12 stimulation sites), encoded by stimulation strength ( $\geqq 2$ stimulation sites), or a combination of the two. The tests indicated that spatial encoding was perceived as more intuitive, but intensity encoding yielded better performance and lower energy expenditure.

The second study investigated the detrimental influence of stimulation artefacts on myoelectric control of prostheses for a wide range of stimulation parameters and two prosthesis control approaches (pattern recognition of eight motion primitives, direct proportional control). Artefact blanking is introduced and discussed as a practical approach to handle stimulation artefacts and restore control performance back to the baseline. This was shown with virtual and applied artefact blanking (pattern recognition on six electromyographic channels), as well as in a practical task-related test with a real prosthesis (proportional control).
\end{abstract}


The information transfer of sensory feedback necessary to master a routine grasping task using electromyographic control of a prosthesis was investigated in another study. Subjects controlled a real prosthesis to repeatedly grasp a dummy object, which implemented two different objects with previously unknown slip and fragility properties. Three feedback conditions (basic feedback on grasp success, visual grasp force feedback, tactile grasp force feedback) were compared with regard to their influence on subjects' task performance and variability in exerted grasp force. It was found that online force feedback via a visual or tactile channel did not add significant advantages, and that basic feedback was sufficient and was employed by subjects to improve both performance and force variability with time. Importantly, there was no adverse effect of the additional feedback, either. This has important implications for other non-functional applications of sensory feedback, such as facilitation of embodiment of prosthetic devices.

The final study investigated the impact of electrocutaneous stimulation on embodiment of an artificial limb. For this purpose, a sensor finger was employed in a rubber-hand-illusion-like experiment. Two independent groups (test, control), were compared with regard to two objective measures of embodiment: proprioceptive drift, and change in skin temperature. Though proprioceptive drift measures did not reveal differences between conditions, they indicated trends generally associated to a successful illusion. Additionally, significant changes in skin temperature between test and control group indicated that embodiment of the artificial digit could be induced by providing sensory substitution feedback on the forearm.

In conclusion, it has been shown that humans can employ electrocutaneous stimulation feedback in challenging closed-loop control tasks. It was found that transition from simple intuitive encodings (spatial) to those providing better resolution (intensity) further improves feedback exploitation. Blanking and segmentation approaches facilitate simultaneous application of electrocutaneous stimulation and electromyographic control of prostheses, using both pattern recognition and classic proportional approaches. While it was found that force feedback may not aid in the mastering of routine grasping, the presence of the feedback was also found to not impede the user performance. This is an important implication for the application of feedback for non-functional purposes, such as facilitation of embodiment. Regarding this, it was shown that providing sensory feedback via electrocutaneous stimulation did indeed promote embodiment of 
an artificial finger, even if the feedback was applied to the forearm. Based on the results of this work, the next step should be integration of sensory feedback into commercial devices, so that all amputees can benefit from its advantages. Electrocutaneous stimulation has been shown to be an ideal means for realising this. Hitherto existing concerns about the compatibility of electrocutaneous stimulation and myocontrol could be resolved by presenting appropriate methods to deal with stimulation artefacts. 



\section{Contents}

Contents vii

1 Introduction $\quad 1$

1.1 Motivation . . . . . . . . . . . . . . . . . 1

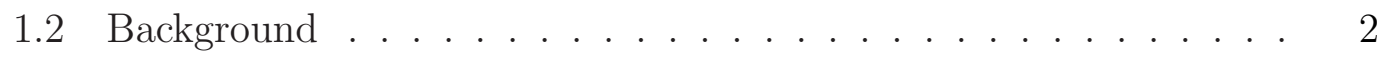

1.2.1 Sensory feedback for prosthetic applications . . . . . . . . 2

1.2.2 Electrical stimulation for sensory feedback . . . . . . . . . 4

1.3 Problem description . . . . . . . . . . . . . . . . . 6

1.4 Outline . . . . . . . . . . . . . . . . . . 7

2 Encoding sensory feedback: Transmitting the information 9

2.1 Methods . . . . . . . . . . . . . . . . . 10

2.1.1 Experimental setup . . . . . . . . . . . . . . . 12

2.1.2 Participants and feedback approaches . . . . . . . . . . 13

2.1.3 Experimental procedure ................ . . 14

2.1 .4 Outcome measures . . . . . . . . . . . . . . 15

2.2 Results . . . . . . . . . . . . . . . . . . . . . 15

2.3 Discussion . . . . . . . . . . . . . . . . . . . . 21

3 Including myocontrol: The challenge of stimulation artefacts 25

3.1 Literature review . . . . . . . . . . . . . . . . . . . . . . 26

3.2 Artefact blanking in pattern recognition . . . . . . . . . . . . 28

3.2.1 Methods . . . . . . . . . . . . . . . . . . 29

3.2.1.1 Pattern Recognition . . . . . . . . . . . . 29

3.2.1.2 Classification Algorithm and Features . . . . . . 29

3.2.1.3 Virtual and Applied Blanking . . . . . . . . . . 31

3.2.1.4 Outcome Measures . . . . . . . . . . . . . . . 32

3.2.1.5 Experimental Setup and Protocol . . . . . . . . 32

3.2 .2 Results . . . . . . . . . . . . . . . 36

3.2.2.1 Impact of Stimulation Artefacts . . . . . . . . . . 36

3.2.2.2 Virtual Blanking . . . . . . . . . . . 36 
3.2.2.3 Applied Blanking . . . . . . . . . . . . . . . 41

3.3 Artefact blanking for proportional control . . . . . . . . . . . . . 42

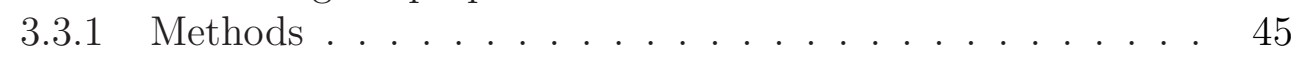

3.3.1.1 Proportional control . . . . . . . . . . . 45

3.3.1.2 Experimental setup and protocol . . . . . . . . 45

3.3.1.3 Outcome measures . . . . . . . . . . . . . . 48

3.3 .2 Results . . . . . . . . . . . . . . . . . . . . 48

3.4 Discussion . . . . . . . . . . . . . . . . . . . . . . . . . . . . . . . . 49

3.4.1 Pattern Recognition . . . . . . . . . . . . . . 49

3.4.2 Proportional Control . . . . . . . . . . . . . 52

4 Full closed-loop myocontrol in routine grasping: The role of feedback $\quad 55$

4.1 Methods ...................... 57

4.1.1 Experimental Setup and Protocol . . . . . . . . . . 58

4.1.2 Participants and conditions . . . . . . . . . . . . . . . 59

4.1 .3 Outcome measures . . . . . . . . . . . . . . 61

4.2 Results . . . . . . . . . . . . . . . . . . . 62

4.3 Discussion . . . . . . . . . . . . . . . . . . . 68

5 Electrocutaneous feedback to facilitate embodiment of prosthetic $\begin{array}{ll}\text { devices } & \mathbf{7 1}\end{array}$

5.1 Methods ......................... 73

5.1 .1 Experimental setup . . . . . . . . . . . . . . 73

5.1 .2 Participants and experimental conditions . . . . . . . 75

5.1 .3 Outcome measures . . . . . . . . . . . . . . 78

5.1 .4 Experimental procedure . . . . . . . . . . . . 79

5.2 Results . . . . . . . . . . . . . . . . . . . 80

5.3 Discussion . . . . . . . . . . . . . . . . . . . 81

6 Summary \& Conclusions $\quad 85$

$\begin{array}{lr}\text { Bibliography } & 89\end{array}$

Appendix A Modular test environment for closed-loop prosthesis control 


\section{Chapter 1}

\section{Introduction}

\subsection{Motivation}

Traumatic or disease-related limb loss has a severe impact on the life of those who have to suffer such misfortune, challenging them both physically and mentally, as well as psychologically. Thanks to modern prosthetics, affected people can - after a certain rehabilitation phase - mostly still live an independent and self-determined life. Recent advancements in this field continue to improve the situation of amputees. Notably, the functional and aesthetic replacement of the lower limb has reached a level that enables wearers of modern leg prostheses to even practice many sports, including extreme sports. Arm and hand function, however, are far more difficult to mimic and replace. Reasons for this can be found in the high complexity of motions and actions we can perform with our hands. This, of course, is made possible by the large number of joints and muscles that are involved in hand motion, as well as the rich and complex sensory feedback available to us.

A variety of devices is commercially available that can at least restore part of the motor function of the human hand and arm, including dexterous artificial hands where a number of joints are individually controllable by the user. There is one thing, however, that is still completely missing, and this is a device that allows the user to feel again.

Natural sensory feedback comprises a number of senses. Sensory cells enable us to feel pressure, vibration, and temperature. Together, they form our sense of touch and allow us to distinguish properties of different materials and objects. Another sense we posses is proprioception, i.e., the sense of where our body parts are relative to each other and how they move. When estimating their limb positions, and especially when planning and executing motions, healthy humans depend on both vision and proprioception [1-3]. Prosthesis users, however, are limited to visual control when it comes to checking if their arm or hand is in the 


\section{INTRODUCTION}

right place and position for a certain action. The lack of sense of touch means that they are not able to 'sense' the coldness of a beer glass, or the warmth of a human touch. Unless they happen to glance at their hand, they may not notice the slipping of the object they are holding, before they hear it crash on the floor. So understandingly, somatosensory feedback is a feature that is highly desired by prosthesis users. Surveys amongst prosthesis users revealed that lack of sensory feedback is an issue and even plays a role in prosthesis abandonment [4]. And when asked about preferences for future improvement of prostheses, a vast majority of participants wished for grasping force feedback and many desired a temperature feedback as well [5].

Providing sensory feedback to amputees would go beyond merely fulfilling prostheses users' desires to 'get back in touch' with the world. A number of studies support the hypothesis that inclusion of feedback may also advance control of prostheses. Functional benefits include greater success at the task of grasping and lifting objects of unknown weight [6]. Mental effort when manipulating fragile objects could be tremendously decreased, while confidence could be boosted. However, not all studies investigating closed-loop prosthesis control support this hypothesis. Hence, this potential benefit is still discussed controversially, and should be subject to further investigation.

Last but not least, it was shown that sensory stimulation, even if not eventrelated, appears to be a promising treatment for phantom limb pain [7], a condition affecting large numbers of amputees, and for which no effective, long-term stable treatment has been found yet [8].

By integrating sensors into prosthetic devices, information on the prostheses' interaction with the environment and other system states like joint angles can be collected. The emergence of flexible, stretchable electronic skins with temperature, pressure and vibration sensing capabilities [9] opens up new possibilities to obtain even more sensory data. Technologically, it is therefore possible to gather the information relevant to restore both the sense of touch and proprioception. Consequently, the next challenge is to find ways to transmit this information to the prosthesis user.

The following sections give an overview of the current academic state of the art in 'feeling' prostheses and different approaches to restore the sense of touch.

\subsection{Background}

\subsubsection{Sensory feedback for prosthetic applications}

The natural sensory feedback from our upper extremities, and especially from our hands, is very rich and complex. Still, a lifetime of experiencing this feed- 
back tought us to derive from it much information on our interaction with the environment, on properties of objects we are handling, and also our hands themselves with no or hardly any effort. For prosthesis users, however, who were just recently deprived of this natural feedback, it does not seem feasible to overload them with overly complex and multilayer artificial sensory feedback. Hence, there is the need to select from the huge pool of possible feedback variables either a single variable, or a subset of few variables. Touch or pressure on the skin [10], grip force [11-15], temperature or information related to proprioception, such as distance of thumb and finger tips (hand opening) [11,12], are counted among the obvious choices and attracted interest in the past. But also other less obvious variables were suggested, such as hand opening/closing speed and muscle activity. Once feedback variables have been selected, a way needs to be found to transmit the relevant information to the prosthesis user. In general, there are two approaches how an artificial sensory feedback can be realized, so-called modalitymatched feedback, and sensory substitution, respectively [16]. Modality-matched feedback exactly mimics the naturally occurring feedback conditions, e.g. pressure is transmitted by mechanical pushers [10], or temperature information is recreated by a heating and cooling element on the skin.

For sensory substitution, on the other hand, the modality of the feedback may change, e.g. pressure information might be encoded in a vibration pattern.

While modality-matched feedback promises to be very intuitive, its implementation is often technologically challenging. Flexibility is far greater for sensory substitution, but interpreting and internalising of this type of feedback also requires greater openness and mental effort on the prosthesis user's side. Both approaches have been investigated for decades, and a number of proposals for prosthetic systems with sensory feedback for the user have been made. These include a myoelctrically controlled prosthesis hook with grip force feedback via a motor-driven pusher [15], a robotic hand where pressure on the fingers is transferred to a pressure cuff around the user's upper arm [17], and a hand prosthesis with a sensing thumb, where pressure on the thumb, vibration and temperature were transferred to the upper arm by a squeezing device, including a vibrator and a Peltier element [18]. The former all represent systems which aim for modality-matched feedback. Sensory substitution systems include myoelectrically controlled hands with electrocutaneous feedback for grip force [13, 14], myoelectrically controlled hands with electrocutaneous feedback on both grip force and hand opening $[11,12]$, and a simple hand and elbow prostheses with electrocutaneous feedback for both grip force and hand opening [11]. 


\section{INTRODUCTION}

\subsubsection{Electrical stimulation for sensory feedback}

Non-invasive electrotactile stimulation as means to provide sensory feedback to prostheses users was proposed as early as the 1970s [11].

The principle of electrocutaneous stimulation is to create an alternating electric field that penetrates the skin and changes the electric membrane potential of the afferent nerve fibres in the skin. A nerve cell's inherent resting membrane potential typically measures around $-0.07 \mathrm{~V}$. When the membrane is slightly depolarised and its potential is increased above a certain threshold, this causes the nerve cell to release its neural transmitters and the electric nerve pulse is passed on. Thus, non-invasive electric stimulation can elicit tactile sensations when a sufficient number of afferent nerve fibres are depolarised by externally applied electric fields.

The fields are induced by superficial electric currents which are applied via bipolar electrodes. These electrodes can either be realised by placing two or more spatially separated electrodes on the skin, or they can take a concentric form, where an inner electrode is surrounded by an outer ring-shaped electrode. Fields generated by spatially separated electrodes spread more and stimulate greater areas of the skin. With concentric designs, electric current and resulting field are more localised, which also leads to more localised sensations as only the afferent nerve fibres in a small area of skin respond. Usually, alternating currents that take the shape of biphasic current pulses are used for electrotactile stimulation, as they are perceived as more comfortable and avoid accumulation of charge in the skin, which can be harmful [19]. Figure 1.1 shows an example of such a pulse train.

In order to change the quality and the strength of the perceived sensation a number of parameters can be tuned, including current amplitude, pulse width, and pulse frequency $[20,21]$. Both quality and strength of the perceived sensation also strongly depend on the location of the stimulation and the distribution and density of sensory cells and nerve fibres in the target skin area. In general, electrotactile stimulation can be perceived as touch, pressure, buzz, vibration, numbness, tingling and pain [21].

Recently, also invasive techniques move to the center of attention, as attempts are made to create human machine interfaces on a neural level [22-27]. Different approaches for implantable electrodes have emerged, such as cuff electrodes, which encase and target a whole nerve bundle, needle electrodes, which pierce the nerve bundle and could target nerve fibres more selectively, or sieve electrodes, which have microscopic holes that allow single nerve fibres to grow into the electrode. However, implantation of invasive electrodes require delicate surgical procedures, and long term usability is still an issue [28]. 


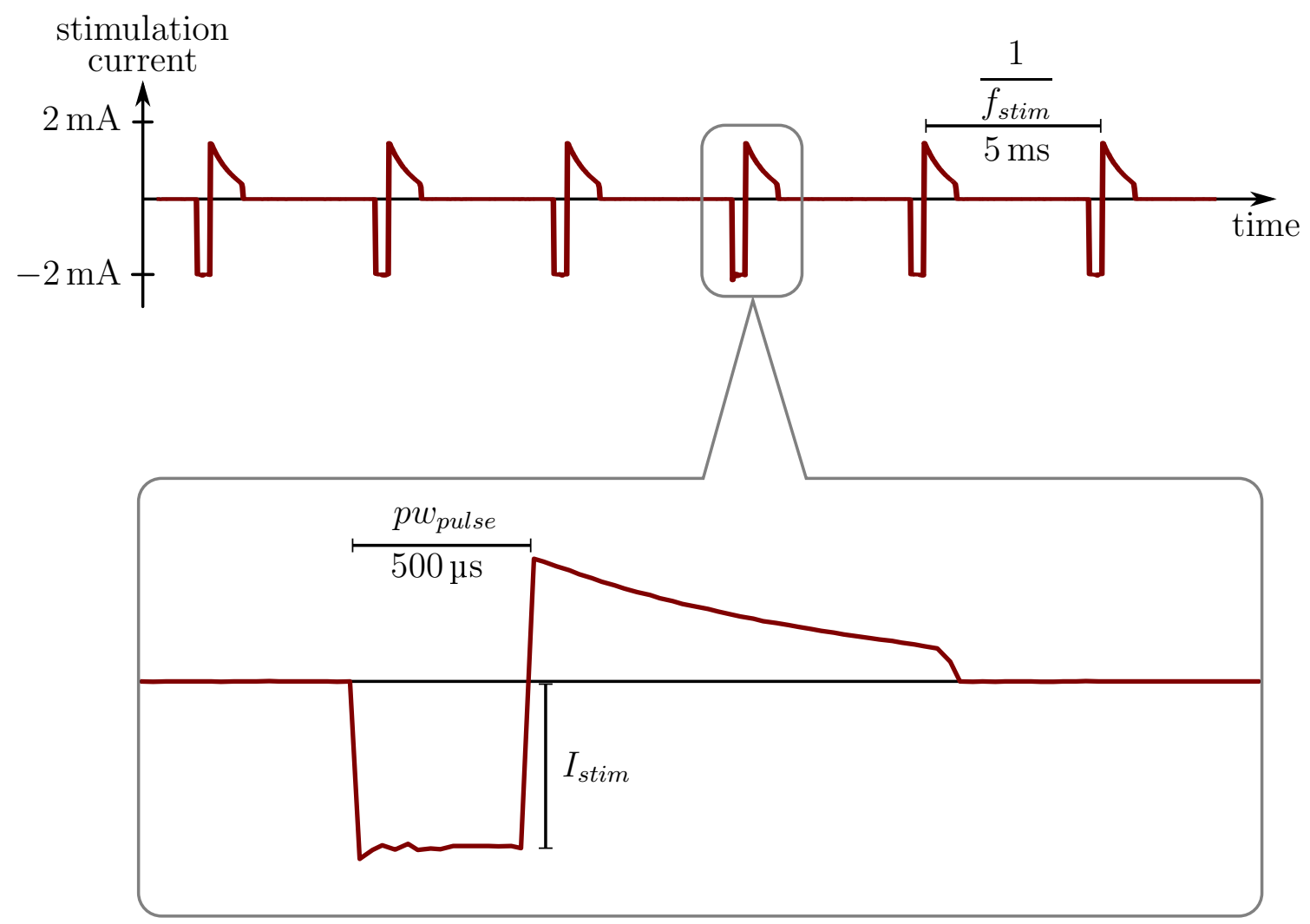

Figure 1.1: Example of a biphasic pulse train used for electrotactile stimulation. Current amplitude $I_{\text {stim }}$, pulse width $p w_{\text {pulse }}$ and pulse frequency $f_{\text {stim }}$ can be changed to modify perceived sensation. 


\section{INTRODUCTION}

\subsection{Problem description}

As indicated, a number of exciting and promising studies investigated direct neural interfaces. However, these require additional surgical steps and long term stability cannot yet be guaranteed. For user acceptance it is furthermore crucial, that the prosthesis system is self-contained for easy donning and doffing. Hence, non-invasive solutions need to be found, so that a large number of affected patients can benefit from the functional and medical advantages of sensory feedback as soon as possible. This thesis is dedicated to investigating a sensory feedback approach for prosthetics that promises a transition to the market in the very near future.

From a user-centred point of view, electrocutaneous stimulation unites many advantages. It consumes less energy than mechanical solutions like vibrators or pushers, thus having a positive effect on battery life, which should be at least eight hours to suffice for daily use of the prosthesis. The technology is noiseless and very compact, and could easily be integrated into the prosthesis socket, which is essential for the self containment of the prosthetic system.

But there are challenges as well. Modern hand and arm prostheses are controlled by myoelectric signals gathered by electrodes which are integrated into the prosthesis socket. With increasing complexity of a prosthetic device with regard to number of joints that should be individually and even simultaneously controllable, there is a need to increase the number of electrodes, and high quality of the control signals has to be guaranteed. With recording and stimulation electrodes both integrated into the prosthesis socket, however, electrical fields are generated in close proximity to the sensitive recording electrodes, resulting in stimulation artefacts which interfere with the recorded control signals. This has detrimental effects on the reliability of the control itself [12], and poses an issue that needs to be handled.

The work underlying this dissertation was dedicated to investigating and understanding these effects and to identify a feasible method to handle this problem. Furthermore, its goal was to choose feedback variables which promise to be most beneficial to the prosthesis user, and identify encoding schemes to transmit the sensory information via electrotactile stimulation. Experimental assessment of possible function benefits, as well as possible facilitation of incorporation of artificial body parts into the body scheme through electrotactile feedback complete the scope of this work. 


\subsection{Outline}

The doctoral project was organised into four independent studies which are reflected in the following chapters. Chapter 2 is dedicated to the encoding of artificial sensory feedback and the identification of suitable electrocutaneous stimulation patterns for information transmission. These aspects were investigated in a study specifically designed to be independent from myoelectric prosthesis control. The results of this study constitute the basis for further studies conducted within the project.

The third chapter presents results of the analysis of the theoretical and practical investigation of the detrimental influence of stimulation artefacts on myoelectric control of hand prostheses, and recommendations for stimulation parameters derived from this. A practical approach to deal with stimulation artefacts that is still compatible with different control strategies is presented to round off this aspect of the project.

The third study was aimed at investigating potential benefits of sensory feedback for prosthesis users when they attempt to train a routine grasping task. The study and its outcome are presented in chapter 4.

Chapter 5 describes the final study of the project, which dealt with the aspect of embodiment of a prosthetic device, i.e., its integration into the body scheme of the user, and in how far electrocutaneous feedback can assist to facilitate embodiment.

The final chapter of the dissertation summarizes the whole doctoral project, and presents the overall conclusions that can be drawn from the work.

Figure 1.2 illustrates the interactions between the four studies, and provides quick access to the corresponding chapters.

For additional information, the interested reader can find in the appendix the description of a flexible test environment developed in Matlab Simulink Realtime Workshop. The goal of this test environment was to be able to easily set up and configure experimental setups to compare different prostheses, feedback systems and feedback encodings under repeatable conditions. It constitutes the fundamental basis for all experimental work done in the course of this project. 


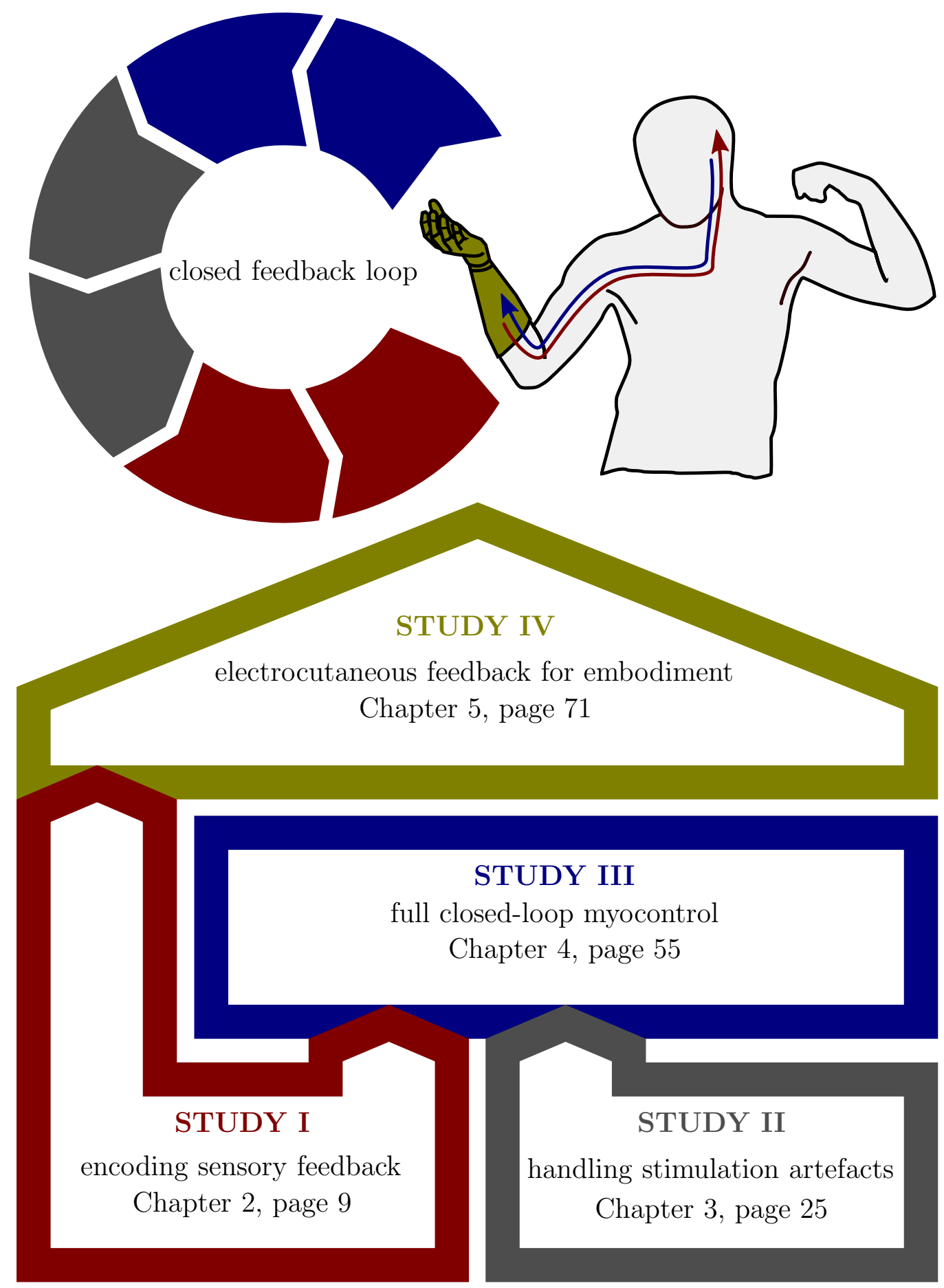

Figure 1.2: Structure of the dissertation and described studies. 


\section{Chapter 2}

\section{Encoding sensory feedback: Transmitting the information}

One of the first issues to be addressed when designing an electrocutaneous feedback for prostheses users is the representation of the the feedback variable in an electrical stimulation pattern. One possible strategy is to take the modality of the feedback variable into account and attempt to create modality-matched feedback. While in theory this is possible with electrocutaneous stimulation when the correct afferents are targeted and activated, it is highly challenging to achieve consistent results across subjects. The difficulty of sharply localising the electric fields in combination with the unknown individual physiologic conditions (skin thickness and conductivity, position of nerve fibres) make it impossible to design a generic stimulation patterns for a specific modality. In practice, unintentional co-activation of neighbouring afferents of a different modality are highly likely. Fortunately it was demonstrated in monkeys, that artificial sensory feedback does not necessarily have to mimick natural feedback in order to be exploitable. Using intracortical microstimulation, Dadarlat et al. created an artificial sensation conveying information about the relative position of the monkey's hand with respect to a target [3]. Although not plausible in the sense of natural feedback, monkeys were still able to make use of this information, demonstrating that a learning-based approach to sensory substitution feedback is highly viable. For this reason, focus is put on feedback encodings that promise good information transmission, rather than closely mimicking natural sensory feedback. This has the additional advantage that once viable feedback encodings were identified, the same method can be applied to any feedback variable, i.e., both natural sensory feedback variables (tactile sensations, proprioception-related), as well as new, artificial feedback variables (system states).

In this study, a virtual pendulum stabilisation task was employed to evaluate four different strategies for tactile feedback. Performance of the subjects while 


\section{ENCODING SENSORY FEEDBACK: TRANSMITTING THE INFORMATION}

stabilising the invisible inverted pendulum was compared to the benchmark performance where the pendulum was visible. Importantly, the comparison of feedback encoding paradigms was decoupled from myoelectric feedforward control, which introduces uncertainty due to the noisy characteristics of EMG. Thus, an important confounding factor could be excluded.

This chapter reuses material previously published in the IEEE Transactions on Neural Systems and Rehabilitation Engineering in a research paper entitled Sensory Feedback in Prosthetics: A Standardized Test Bench for Closed-Loop Control by S. Dosen, M. Markovic, C. Hartmann, and D. Farina (c) 2014 IEEE [29]. Parts of the sections 2.1-2.3 (Methods, Results, and Discussion) are textually based on passages from the corresponding sections of the paper.

\subsection{Methods}

When designing a feedback encoding paradigm for electrocutaneous stimulation, a number of parameters can be exploited to modulate the stimulation and encode feedback variables. Firstly, there are the three basic stimulation properties characterising the stimulation pulse train, which can each be modulated separately (compare also section 1.2.2). These are stimulation current amplitude $I_{\text {stim }}$, stimulation pulse width $p w_{\text {pulse }}$, and pulse frequency $f_{\text {stim }}$. While modulation of $I_{\text {stim }}$ and $p w_{\text {pulse }}$ both change the intensity of the perceived sensation, frequency modulation has less effect on the perceived intensity, but is clearly perceived in its temporal aspects. Secondly, by using multiple electrodes, spatial feedback encodings can be designed. Here, information is transmitted by activation or deactivation of single electrodes, effectively shifting or changing the location of the perceived sensation.

In 1977, Szeto and Lyman compared ten different feedback encodings, including intensity, frequency and spatial encodings, in a random tracking task [30]. Further factors were location of stimulation (abdomen or upper arm), as well as general shape of the stimulation pulse train (monophasic or biphasic). As the stimulation location is limited to the forearm in order to maintain self-containment of prosthetic systems, and biphasic stimulation is nowadays preferred over monophasic stimulation for various reasons, findings regarding these factors could be ignored for the design of feedback for this study. Regarding the other factors, results of the study clearly indicated that subjects performed best with spatial encoding, and that seven electrodes were better than five. When using a single electrode to convey the feedback information, it was found that frequency modulation was superior to intensity modulation, and that using a frequency range of $1 \mathrm{~Hz}$ to $15 \mathrm{~Hz}$ yielded better results than with frequency modulated between $20 \mathrm{~Hz}$ and $100 \mathrm{~Hz}$. 
A result somewhat contradictory to these findings was obtained recently by Paredes et al., who investigated the influence of the pulse frequency within the scope of closed-loop control more comprehensively [31]. They used a compensatory tracking experiment where the tracking error was provided by intensity modulated electrocutaneous stimulation. The tracking performance for a range of pulse frequencies $(5 \mathrm{~Hz}$ to $100 \mathrm{~Hz}$ ) as well as error sampling rates was compared, and found to deteriorate significantly when stimulation was delivered at less than $50 \mathrm{~Hz}$. Interestingly, decreasing the error sampling rate from $100 \mathrm{~Hz}$ to $5 \mathrm{~Hz}$ did not adversely influence performance when stimulation pulses were still delivered at a high frequency $(100 \mathrm{~Hz})$.

It was speculated that it is important for stimulation frequency to be sufficiently high for good perception of the value of the intensity-modulated feedback variable. This limits the range of stimulation frequencies that are both distinguishable and at the same time ensure that feedback is delivered with sufficient perception quality.

That Szeto and Lyman found low-range frequency modulation to be superior to intensity modulation could be influenced by the fact that intensity-modulated feedback was delivered at a pulse rate of only $20 \mathrm{~Hz}$, which was found to be too low by Paredes et al.

For these reasons a constant stimulation frequency $f_{\text {stim }}$ was chosen for all proposed feedback encoding paradigms in this study, while information was either encoded by intensity modulation, spatial distribution across several stimulation sites, or a combination of these two. While it was previously found that subjects performed better using spatial encodings [30], an alleged disadvantage of using a limited number of electrodes is that it also limits the resolution of the feedback signal. Therefore, an additional aim of the current experiment was to test if a gradual transition from spatial feedback interfaces to more demanding single electrode interfaces and intensity encoding can be achieved with training.

For this purpose, the different encodings were compared in the context of a closedloop control task. Subjects first performed the task using the most intuitive feedback interfaces, whereupon more demanding feedback approaches were gradually introduced. As a control task, stabilisation of an invisible inverted pendulum was chosen. The task was preventing the pendulum from falling over, by means of 'pushing' it back to the vertical position using a joystick. Deviation from the vertical position could be derived from the electrocutaneous stimulation signals. Number of pendulum falls during a fixed-length trial, as well as average deviation from vertical were compared for all four encodings in order to evaluate the feasibility of the different approaches. 


\section{ENCODING SENSORY FEEDBACK: TRANSMITTING THE INFORMATION}

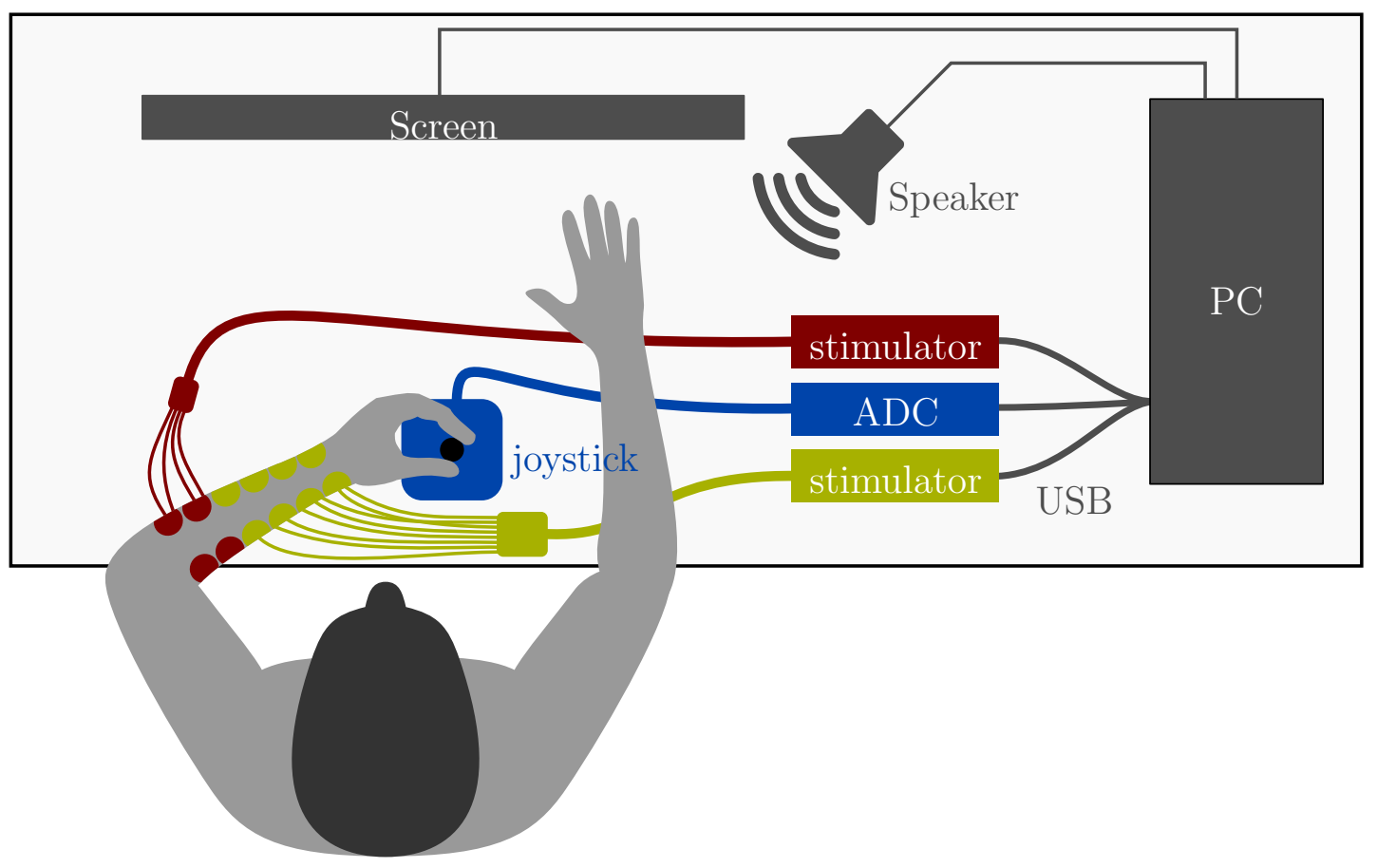

Figure 2.1: The subject uses a joystick to influence the inclination of a virtual inverted pendulum. Depending on the feedback condition, either a visual representation of the pendulum is shown on the screen, or pendulum inclination is encoded in an electrocutaneous stimulation pattern. Dropping of the pendulum (pendulum reaches horizontal) is indicated by a beep from the speaker.

\subsubsection{Experimental setup}

Figure 2.1 shows a schematic view of the experimental setup. The task for the subject was to stabilize a simulated planar inverted pendulum using an analogue joystick, while the feedback about the pendulum inclination was provided via electrotactile stimulation. The joystick inclination was proportional to the torque at the base of the pendulum. To deliver the electrotactile feedback, 12 concentric electrodes (CODE501500, Ø $40 \mathrm{~mm}$, Spes Medica, Italy) were positioned along the dorsal and ventral side of the forearm, six electrodes on each side. The stimulation was provided using two 8-channel medical stimulation units (RehaStim, Hasomed GmbH, Germany). The side of the forearm (dorsal, ventral) to which the stimulation was delivered denoted the side toward which the pendulum was inclined (left, right), and the amount of inclination was coded using spatial and/or intensity encoding.

The modular test environment described in appendix A was used to implement 
the experimental setup, provide the control task, and record experimental data.

\subsubsection{Participants and feedback approaches}

Six subjects ( $30 \pm 5$ years) participated in the experiment after signing informed consent. Each subject performed the task consecutively using all four feedback approaches.

The following configurations were tested:

- Spatial encoding with $2 \times 6$ channels $(12 \mathrm{CH}-\mathrm{S})$.

The $n$-th electrode on the ventral/dorsal side was activated if the inclination angle ${ }^{1}$ was within the interval $\left[ \pm \frac{(n-1)}{6}, \pm \frac{n}{6}\right], n \in\{1,2, \ldots 6\}$, otherwise it was off. Consequently, only one electrode was active at a time.

- Combined spatial and intensity encoding with $2 \times 4$ channels (8CH-SI). The $n$-th electrode was activated if the inclination angle was within the interval $\left[ \pm \frac{(n-1)}{4}, \pm \frac{n}{4}\right], n \in\{1,2,3,4\}$. In addition, the stimulation intensity of the currently active electrode was modulated, linearly mapping the corresponding interval of inclinations to the entire dynamic range of the electrode.

- Intensity encoding with $2 \times 4$ channels $(8 \mathrm{CH}-\mathrm{I})$.

The stimulation intensity was modulated proportionally to the absolute inclination angle on all four electrodes simultaneously, linearly mapping the full interval of inclination to the entire dynamic range of each electrode. Ventral electrodes were activated when inclination was positive, dorsal electrodes were activated when inclination was negative.

- Intensity encoding with $2 \times 1$ channel $(2 \mathrm{CH}-\mathrm{I})$.

Identical to $8 \mathrm{CH}-\mathrm{I}$, but using only one electrode at each side of the forearm.

A summary of the feedback paradigms is given in table 2.1. For the spatial encoding (12CH-S), the stimulation at each electrode was constant and equal to $S T+0.5 R$, with $R=(P T-S T)$, where $S T$ and $P T$ are the sensation and pain thresholds, respectively. For the intensity encoding, the dynamic range was determined as $[S T+0.1 R, S T+0.8 R]$. The $S T$ was estimated for each electrode using the ascending method of limits [32]. PT was determined by continuing to increase stimulus strength until subjects reported uncomfortable or stinging sensations. Pulse width modulation was employed to regulate the stimulation

\footnotetext{
${ }^{1}[-1,1]$ is the normalised signal range for the inclination angle within the test environment. Inclination of \pm 1 corresponds to $\pm 90^{\circ}$ physical inclination (horizontal), 0 corresponds to $0^{\circ}$ physical inclination (vertical).
} 


\section{ENCODING SENSORY FEEDBACK: TRANSMITTING THE INFORMATION}

Table 2.1: Properties of suggested feedback encoding paradigms

\begin{tabular}{ccccr}
\hline & spatial & intensity & $\begin{array}{r}\text { number of } \\
\text { electrodes }\end{array}$ & $\begin{array}{r}\text { range per } \\
\text { electrode }\end{array}$ \\
\hline $12 \mathrm{CH}-\mathrm{S}$ & $\checkmark$ & & $2 \times 6$ & $16.7 \%$ \\
$8 \mathrm{CH}-\mathrm{SI}$ & $\checkmark$ & $\checkmark$ & $2 \times 4$ & $25 \%$ \\
$8 \mathrm{CH}-\mathrm{I}$ & & $\checkmark$ & $2 \times 4$ & $100 \%$ \\
$2 \mathrm{CH}-\mathrm{I}$ & & $\checkmark$ & $2 \times 1$ & $100 \%$ \\
\hline
\end{tabular}

Column 'range per electrode' gives the range of absolute pendulum inclination that is mapped to a single electrode

intensity, while the current amplitude was kept constant at $3 \mathrm{~mA}$ and frequency at $100 \mathrm{~Hz}$.

\subsubsection{Experimental procedure}

The experiment included two sessions performed on two consecutive days. During the first introductory session, which lasted $30 \mathrm{~min}$ to $45 \mathrm{~min}$, the subjects were introduced to the task. They stabilized the pendulum using visual feedback (i.e., visual representation of pendulum on the screen), simultaneous visual and electrotactile feedback (2CH-I) and only electrotactile feedback. In the main testing session, the subjects performed the pendulum stabilisation task using feedback interfaces in the following order: $12 \mathrm{CH}-\mathrm{S}, 8 \mathrm{CH}-\mathrm{SI}, 8 \mathrm{CH}-\mathrm{I}$ and $2 \mathrm{CH}-\mathrm{I}$. The order reflected the expected intuitiveness of the feedback encodings. The subjects started with the most intuitive interface (simple spatial encoding) and finished with the most difficult one (intensity encoding, two electrodes only). Intensity encoding is more demanding for the subjects, since they have to discriminate a continuously changing sensation, but it also implements a finer resolution of information transmission. Pilot tests indicated that the selected challenging control task (i.e., stabilising an unstable system) would be very difficult if not impossible for the subjects if they started with the intensity encoding interface. As mentioned earlier, the aim of this protocol was to test if the aforementioned gradual transition between the feedback interfaces would allow the subjects to eventually accomplish the task and even exploit the inherent advantage of the intensity encoding. Eleven 90-second trials were performed in each condition. When the pendulum fell over (inclination angle $<-90^{\circ}$ or $>90^{\circ}$ ), this was indicated by a short beep sound. Immediately, the pendulum position was reset (inclination 
zero), a low destabilizing impulse was delivered to the pendulum, and the trial continued.

\subsubsection{Outcome measures}

As a measure for the subject performance in the task, the number of falls and the average absolute deviation of the pendulum from vertical were computed over each trial. Total energy of the control signal was computed as the surface below the rectified control signal normalized by $C_{M A X} \cdot T$, where $C_{M A X}$ is the maximum possible control value (here, $C_{M A X}=1$ ), and $T$ is the trial duration (here, $T=90 \mathrm{~s}$ ). Average time between two successive falls of the pendulum was determined to compare the performance of $2 \mathrm{CH}-\mathrm{I}$ in introductory versus test session. When computing the average performance, the first five trials in each condition were regarded as training, and they were not used in the calculation. For statistical analysis Friedman ANOVA and a Tukey's honestly significant difference post-hoc test were applied. Significance threshold was set to $p<0.05$. For comparison of two specific conditions, Wilcoxon signed-rank test was used. Numerical results are reported in the text as mean \pm standard deviation.

\subsection{Results}

Two example trials of the pendulum stabilisation recorded from the same subject using visual (introductory session) and electrotactile (8CH-I, test session) feedback are shown in Figs. 2.2 and 2.3. The subject successfully stabilized the pendulum for $60 \mathrm{~s}$ with small deviations from the vertical. The performance was similar between the feedback types, but interestingly, the control strategy was very different. When visual feedback was available, the subject continuously modulated the control input (joystick inclination). With electrotactile feedback, however, the control was intermittent, i.e., the subject generated short impulses separated by the intervals of zero control action. Such intermittent control was used consistently by all subjects in electrotactile conditions. The transition from the continuous to intermittent control when switching from visual to electrotactile feedback was observed in four subjects. In the two remaining subjects the transition was not as clear, since they tended to control impulsively even with the visual feedback. The change in the nature of control was reflected by the average normalized energy of the control signal, which decreased from $30 \% \pm 11 \%$ for the visual to $20 \% \pm 10 \%$ (12CH-S), $17 \% \pm 11 \%$ ( $8 \mathrm{CH}-\mathrm{SI}, p<0.05), 16 \% \pm 9 \%$ (8CH-I, $p<0.05$ ), and $23 \% \pm 11 \%$ (2CH-I) for the electrotactile conditions.

The summary results for the pendulum stabilisation task using four electrotactile feedback interfaces are given in Figs. 2.4- 2.6. The subject performance 

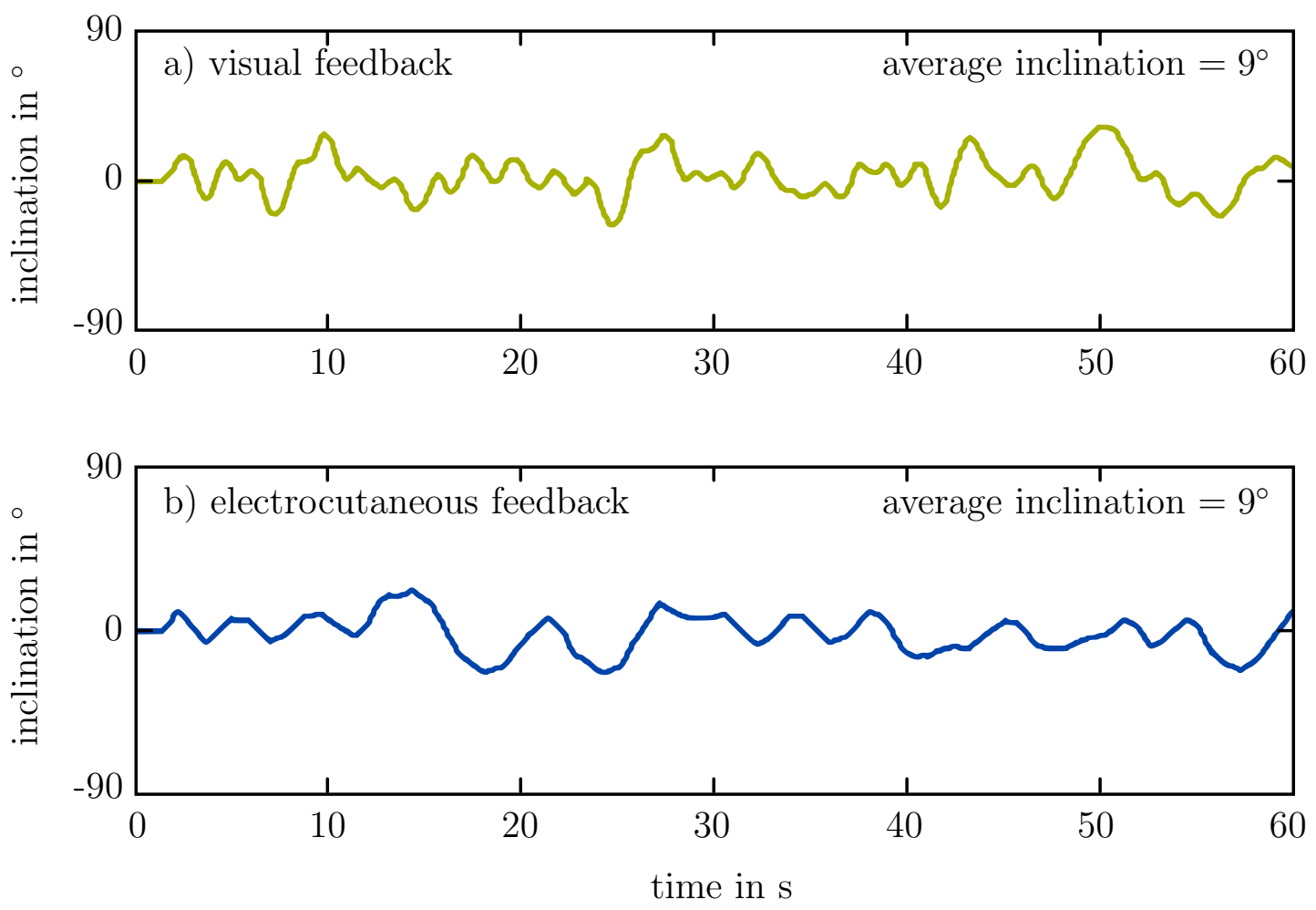

Figure 2.2: Inclination of pendulum in two exemplary trials of stabilisation task with a) visual feedback, and b) electrotactile 8CH-I feedback. Performance was similar with both feedback types. Here, the average inclination across trials was $9^{\circ}$ for both feedback types. Adapted from [29], (C) 2014 IEEE. 

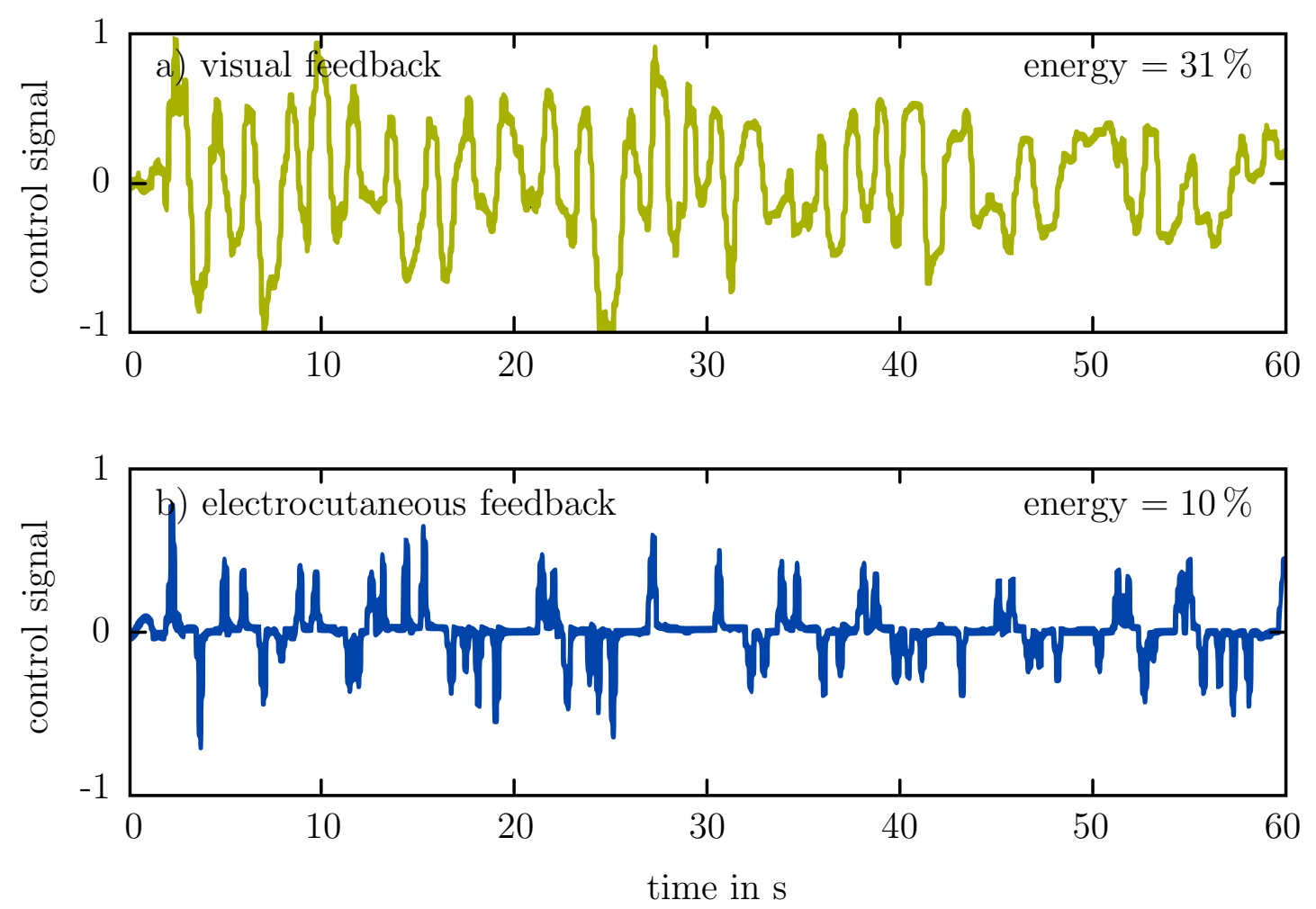

Figure 2.3: Control signal (joystick input) during two exemplary trials of stabilisation task with a) visual feedback, and b) electrotactile 8CH-I feedback. The control strategy was very different for the two feedbacks. While with visual feedback the majority of subjects employed smooth control signals, with electrocutaneous feedback the subjects controlled impulsively. Adapted from [29], (C) 2014 IEEE. 


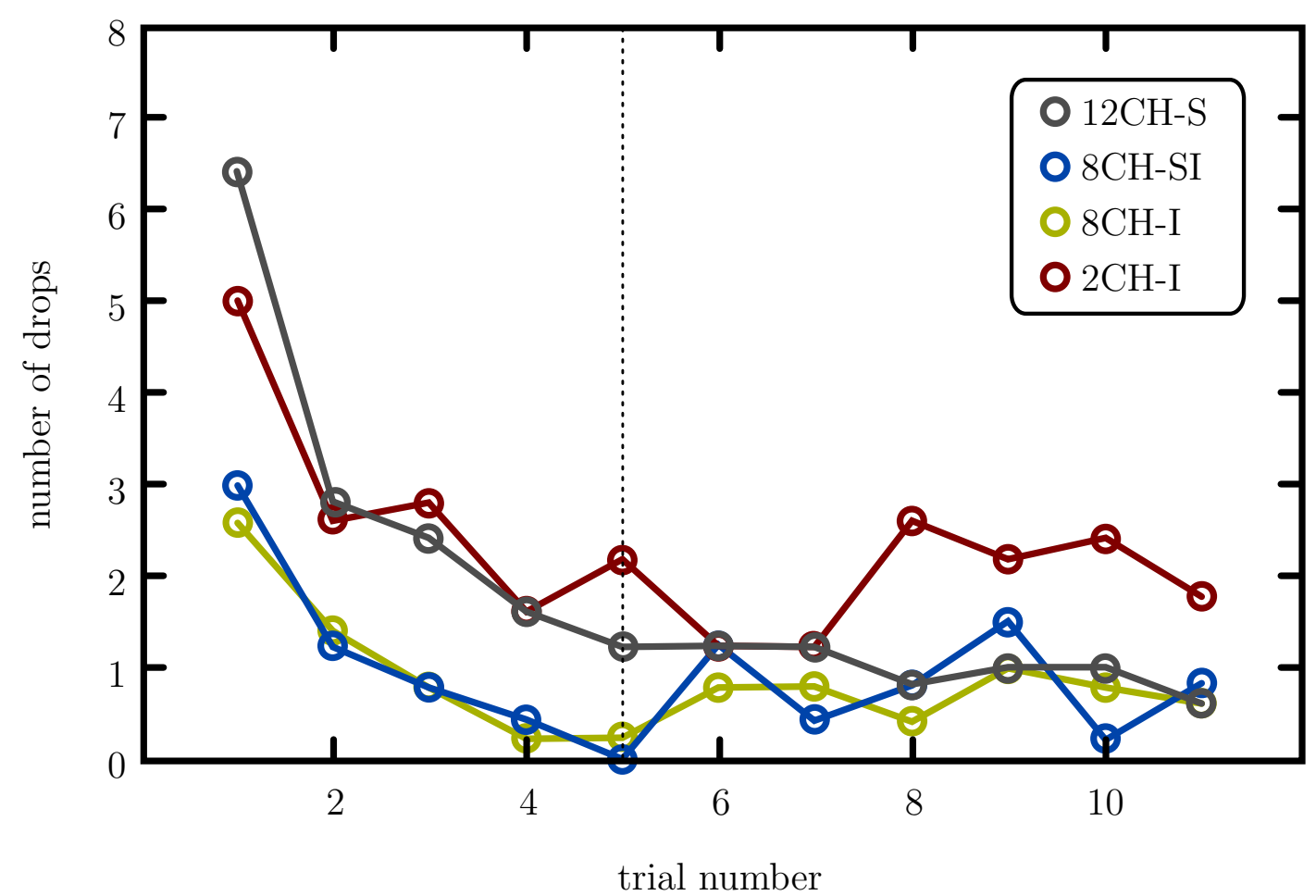

Figure 2.4: Average number of falls (inclination $<-90^{\circ}$ or $>90^{\circ}$ ). There was a trend for performance improvement in the first few trials. After the fifth trial, the performance was more stable. Note that only the mean values are given to reduce the clutter. Adapted from [29], (c) 2014 IEEE. 


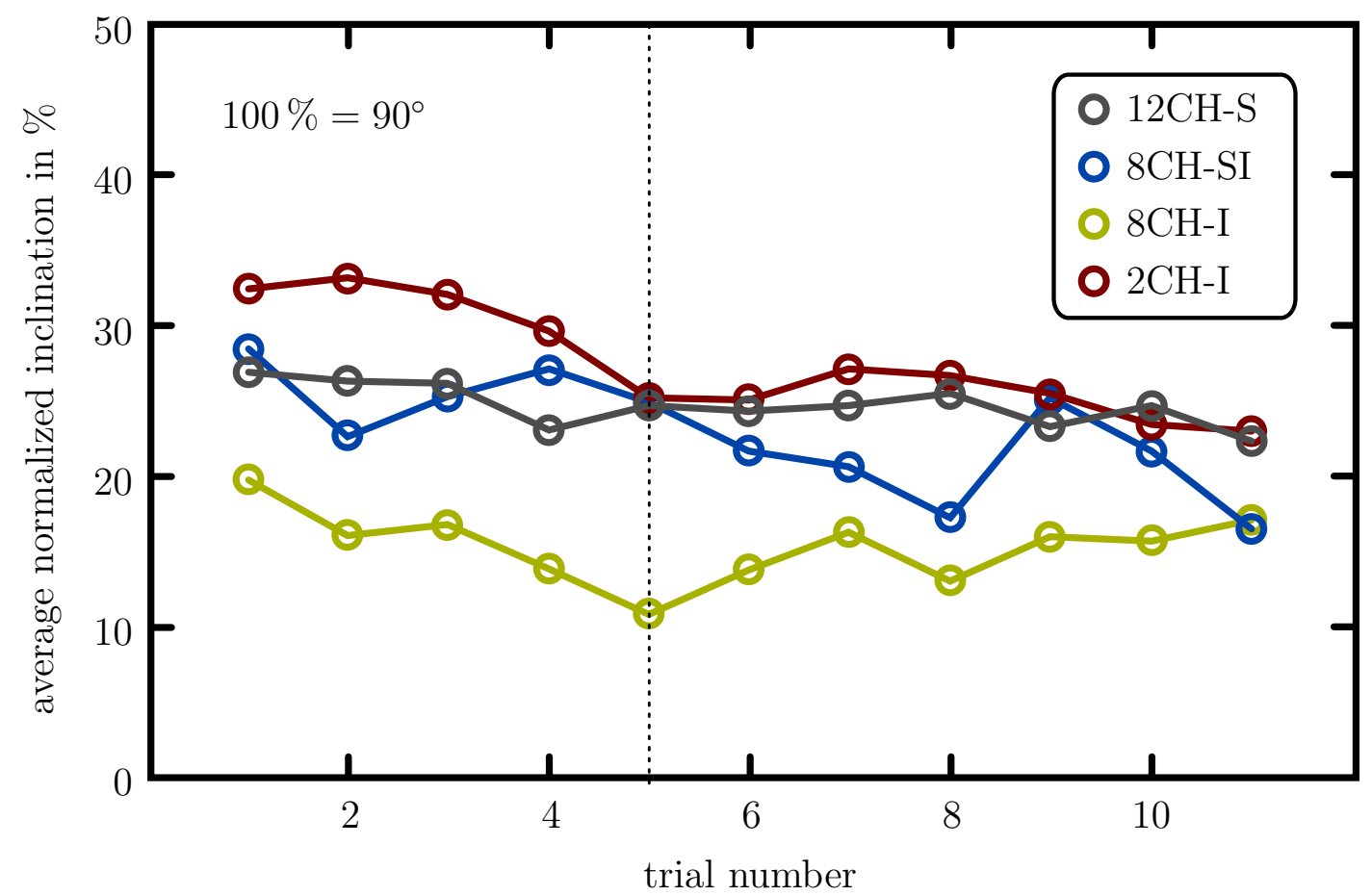

Figure 2.5: Average pendulum inclination (normalised). There was a slight trend for performance improvement in the first few trials. After the fifth trial, the performance was more stable. Note that only the mean values are given to reduce the clutter. Adapted from [29], (C) 2014 IEEE. 


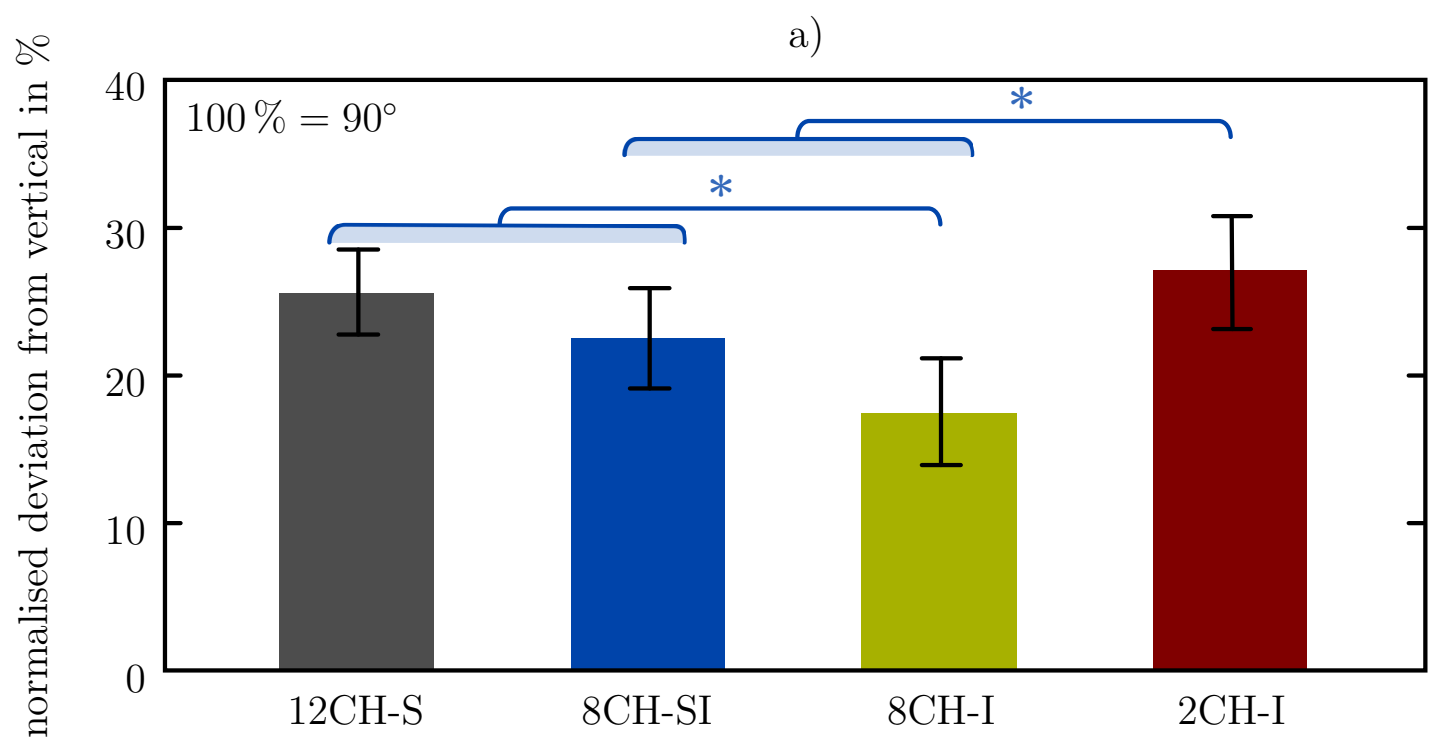

b)

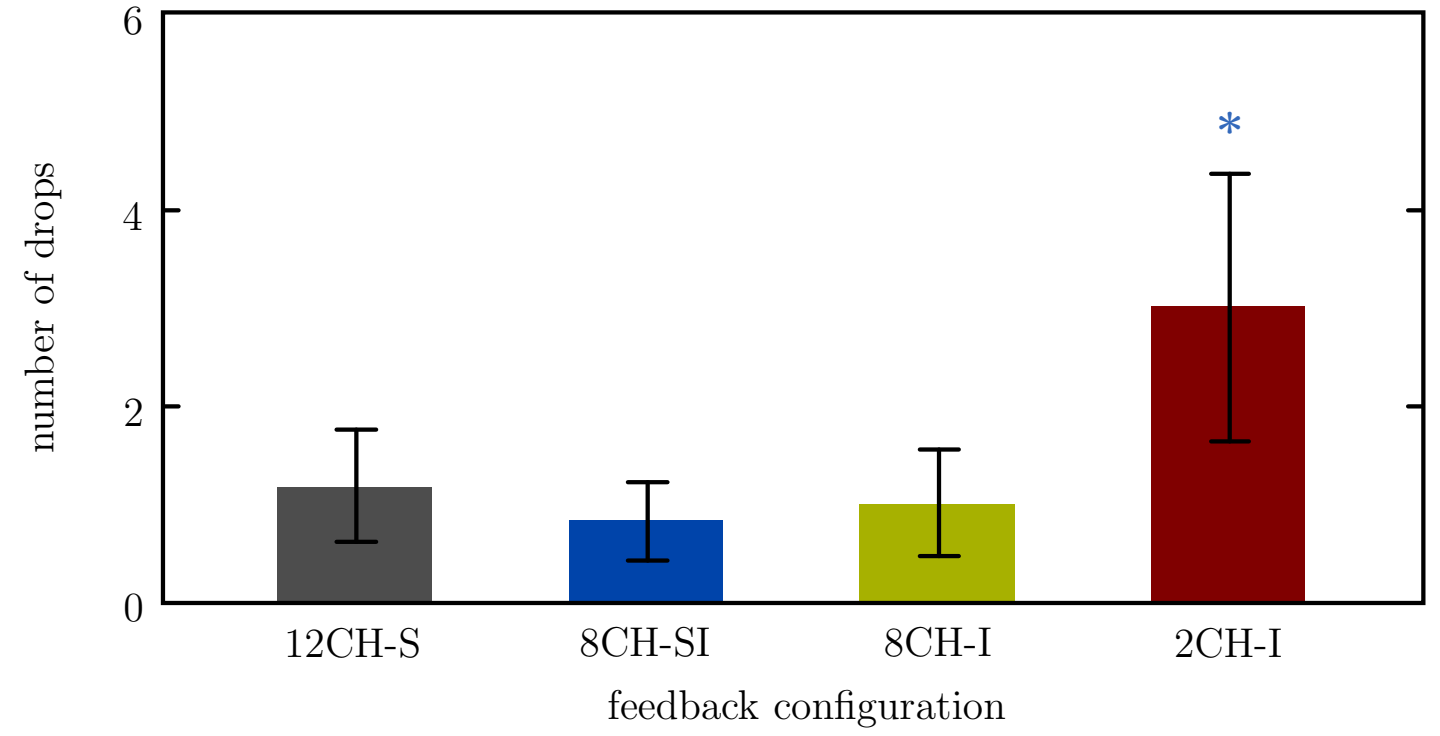

Figure 2.6: Results of the pendulum stabilisation task across conditions (mean \pm standard error): (a) average deviation from vertical, and (b) average number of falls. Best and worst performance resulted when $8 \mathrm{CH}-\mathrm{I}$ and $2 \mathrm{CH}-\mathrm{I}$ feedback configurations were used, respectively. Statistically significant differences are indicated by an asterisk $(*)$. Adapted from [29], (c) 2014 IEEE. 
showed a trend of improvement in the first few trials (Figs. 2.4 and 2.5), with a faster drop in the number of falls (Fig. 2.4). However, after the fifth trial, the performance became more stable. Overall, the best and the worst results were obtained using the intensity encoding, i.e., $8 \mathrm{CH}-\mathrm{I}$ and $2 \mathrm{CH}-\mathrm{I}$, respectively (Fig. 2.6). More specifically, in terms of keeping the pendulum dynamically stable, the interfaces $12 \mathrm{CH}-\mathrm{S}, 8 \mathrm{CH}-\mathrm{SI}$ and $8 \mathrm{CH}-\mathrm{I}$ resulted in a similar performance, and they were all superior to $2 \mathrm{CH}-\mathrm{I}$ (Fig. $2.6 \mathrm{~b}$ ). Although the average deviations were similar for $12 \mathrm{CH}-\mathrm{S}$ and $2 \mathrm{CH}-\mathrm{I}$ (Fig. $2.6 \mathrm{a}$ ), the spatial interface still resulted in significantly less falls per trial (Fig. 2.6 b). The interface $8 \mathrm{CH}-\mathrm{I}$ led to the most precise control (Fig. 2.6 a), i.e., the subjects were most successful in minimizing the pendulum oscillations when using this feedback configuration. Also, this configuration was characterized with significantly lower energy of the control signal compared to $12 \mathrm{CH}-\mathrm{S}$ and $2 \mathrm{CH}-\mathrm{I}$. The subject control actions were therefore better timed and/or graded.

In the introductory session, all the subjects experienced the control of the pendulum using electrotactile feedback with only two channels (2CH-I) as very challenging, and this was also reflected in the poor performance. They had almost no control of the pendulum movements, and thereby it would fall within a few seconds. However, in the test session, the performance with the same feedback interface (2CH-I) improved substantially and the improvement was immediate. The average time between falls for $2 \mathrm{CH}-\mathrm{I}$ in the introductory session was $10 \mathrm{~s} \pm 5 \mathrm{~s}$ and it increased threefold $(32 \mathrm{~s} \pm 29 \mathrm{~s})$ already for the first five trials with the same interface in the testing session.

\subsection{Discussion}

The comparison of feedback interfaces demonstrated that the intensity encoding could be superior to spatial and combined spatial-intensity encoding. Spatial encoding is intuitive, since the subjects can easily perceive and discriminate the feedback information, but the spatial code is inherently discrete and therefore provides a low resolution. On the other side, the intensity encoding provides a virtually continuous representation of the system state. When subjects are trained and therefore familiar enough with the system dynamics, they can exploit the higher resolution of the intensity modulation to improve the control. However, the intensity modulated information is harder to perceive correctly, and therefore in order to be effective, it has to be properly amplified. When only one stimulation channel (2CH-I) was used instead of four ( $8 \mathrm{CH}-\mathrm{I})$, the performance had changed from overall best to overall worst, although in both cases the same encoding was used to deliver the same information. The only difference was the total area of the skin that was stimulated. Finally, in the case of the combined 


\section{ENCODING SENSORY FEEDBACK: TRANSMITTING THE INFORMATION}

encoding, the spatial information was again easy to interpret, but the intensity was still modulated using only one electrode. It was therefore difficult for the subject to exploit the latter and improve the control (8CH-SI versus $8 \mathrm{CH}-\mathrm{I}$ ).

In the current experiment, spatial and intensity encoding, as well as a combination of these was chosen. Frequency modulation could have been also used to communicate the information, albeit taking into account certain limitations regarding the feasible ranges for $f_{\text {stim }}$ [31], as discussed previously . It would be relevant to re-address the comparison between these two methods, especially since there are psychometric and task-related studies implying that human subjects might be more sensitive to changes in the pulse rate [30,33].

The current study provides in any case optimistic implications for the potentials of electrotactile feedback in system control. Namely, it demonstrated that the subjects could successfully accomplish a demanding control task, i.e., managing an unstable system, although they used a nonconventional source of feedback, i.e., a cutaneous sensation communicating the feedback variable (pendulum angle). However, the tests also revealed that the control strategy could change substantially when switching from visual to electrotactile feedback. In addition, they demonstrate a potential training paradigm for electrotactile system control. In the introductory session, the subjects were unable to achieve any meaningful control using the most challenging electrotactile feedback configuration (2CH-I). They also reported general confusion and characterized the task as too difficult. However, after being introduced to the feedback interfaces gradually, starting with the most intuitive one (12CH-S), the subjects could handle the most difficult configuration (2CH-I) substantially better when using it for the second time, i.e., at the end of the test session. Next, designing the closed-loop prosthetic systems can directly benefit from understanding and integrating the motor control strategies of the prosthesis users, especially since they might be very different from the conventional control with visual feedback as revealed by the current experiment. Finally, the experiments demonstrated that in a trained user, the properly amplified intensity encoding allows higher precision. Therefore, a feedback interface for a prosthesis can be envisioned combing the encoding methods: the same multichannel electrode array could be used with the spatial encoding (intuitive, low effort) and then switched by the user into the more demanding but also potentially more precise intensity encoding when necessary (e.g., handling a sensitive object).

Such an approach for combined spatial and intensity encoding was developed for use in the fourth study, which is described in chapter 5. For this, the findings described above were exploited as follows. An artificial sensor finger capable of detecting touch with both good spatial resolution and intensity resolution was used. Combined intensity and spatial encoding was adopted to convey two aspects of the touch sensation. Primary information on the touch location on the 
finger was encoded using intuitive spatial encoding, while the secondary aspect of the touch, i.e., the strength of the touch, was encoded using an intensity encoding. This way, once subjects had accustomed to the more obvious aspect of touch location, they could start exploiting the secondary information. Furthermore, in the third study, the findings above were exploited for designing a multi-channel intensity-coded feedback for a full closed-loop prosthesis control task (cf. chapter 4). In particular, the result that the perceptibility of intensity encoding could be improved by displaying the feedback on more than one stimulation site was adopted to design a well-perceivable, high resolution feedback about grasping force.

In the feedback encoding study presented above, a simplified control interface had been used in order to eliminate the confounding factor of accuracy of myoelectric control. However, once suitable feedback encoding paradigms were identified, the next important step towards closed-loop prosthesis control was to consider the feedforward part of the control loop. Specifically, the acquisition and conditioning of EMG in the context of simultaneous electrocutaneous stimulation had to be addressed with due regard to stimulation artefacts. The following chapter is dedicated to illustrating the influence of electrical stimulation artefacts in the context of EMG control for prosthetic applications, and approaches for artefact handling are suggested and evaluated. Together with the findings regarding the feedback encoding, this forms the basis for closed-loop control of prostheses using electrocutaneous stimulation. 
2. ENCODING SENSORY FEEDBACK: TRANSMITTING THE INFORMATION 


\section{Chapter 3}

\section{Including myocontrol: The challenge of stimulation artefacts}

Self-containment of a prosthetic device is an important feature for user acceptance of the device [34]. In order to maintain self-containment, it is necessary to integrate any feedback systems within the prosthesis or prosthesis socket. For electrocutaneous stimulation, this is easily achievable, as stimulation electrodes can be very flat, small, and even flexible. However, as a consequence, the stimulation electrodes will be located on the residual limb of the amputee, close to the recording sites for electromyographic (EMG) signals in myocontrolled prostheses. When electrocutaneous stimulation is applied close to the EMG recording sites, stimulation artefacts appear on the EMG signals.

This chapter addresses this issue.

Large parts of the work presented here were previously published in the IEEE Transactions on Neural Systems and Rehabilitation Engineering in a research paper entitled Closed-loop control of myoelectric prostheses with electrotactile feedback: Influence of stimulation artifact and blanking in the IEEE Transactions on Neural Systems and Rehabilitation Engineering by C. Hartmann, S. Došen, S. Amsuess, and D. Farina (C) 2015 IEEE [35]. The introductory text as well as section 3.1 contain material taken from section Introduction of the original paper. Section 3.2 and subsections are textually based on sections Methods and Results. Section 3.4.1 corresponds to section Discussion of the paper. Section 3.3 of this chapter adds to the previously published material a supplemental study on the influence of electrocutaneous stimulation during direct proportional control of prostheses during task-based usage of a real prosthesis. Discussion of the results is expanded accordingly. 


\section{INCLUDING MYOCONTROL: THE CHALLENGE OF STIMULATION ARTEFACTS}

As demonstrated in the previous chapter, electrocutaneous stimulation to provide sensory feedback to amputees is a technologically promising approach featuring many advantages such as noiseless information transmission, low power consumption, and - as mentioned before - good integrability into the socket. However, the electrical field generated during the stimulation interferes with the EMG signals that are used for prosthesis control. In the worst case scenario, the amplifier circuit of the measuring equipment is saturated by the stimulation spike, rendering measurements completely unusable until the electronics discharge with time. Even if saturation can be avoided, stimulation artefacts occur, which can have at least the same magnitude as the voluntary EMG signal. Since the EMG signal is used for decoding the user's intention in the control of the prosthesis, the artefacts are likely to be detrimental for the control accuracy.

This study investigates the impact of the stimulation artefact on both classic proportional prosthesis control as well as classification-based control approaches, and proposes various types of blanking procedures as practical strategies to recover performance. For this purpose, two experiments were designed. In an applied grasping task which was tailored to suit the possibilities of a simple proportionally controlled prosthesis. Two bipolar EMG channels controlled opening and closing of the hand. Task execution success was compared under three conditions: a control condition (benchmark), where no electrocutaneous stimulation was present, a condition, where stimulation was applied and potentially detrimental for control, and a condition where a blanking and mirroring approach was used to recover the EMG signals contaminated by stimulation artefacts.

In another experiment dedicated to a classification scenario, six bipolar EMG channels were used to discriminate eight motions and grips, including a natural rest condition. Three methods for segmenting and blanking signals contaminated by electrical stimulation artefacts in the data preparation for the classification of the motions are proposed and compared to each other.

\subsection{Literature review}

It is already known that size and shape of the stimulation artefacts are influenced by many factors. According to Mandrile et al. the distance between stimulation and recording site, inter-electrode distance of recording electrodes, and the level of stimulation current are important factors [36]. But also electrical properties of the skin and EMG amplifier configuration play a role. For example, the configuration of the amplifier's integrated low pass filter influences the appearance of the stimulation artefacts. Using a lower cut-off frequency reduces the artefact amplitude, but at the same time prolongs the artefact. A number of studies address 
the stimulation and measurement setup and propose guidelines which decrease the risk of saturating the EMG amplifier.

It was recommended that stimulation electrodes should be placed as far as possible from EMG recording sites, distance between stimulation anode and cathode should be short, and EMG electrodes should not be placed between these two $[37,38]$. In the context of direct electric nerve stimulation, Almström et al. demonstrated that bipolar balanced stimulation reduces artifacts with respect to monopolar waveforms [37], as the former avoids residual polarization of electrodes or tissue $[37,39]$. However, even though the artefacts can be decreased by implementing an appropriate experimental setup, they cannot be avoided completely. The persisting problem of interference between the electrical stimulation and EMG has been addressed in the related field of EMG-triggered functional electrical stimulation (FES), where higher intensity electrocutaneous stimulation is used to elicit a muscle response. In this case, the stimulation artefact and the M-wave generated by the stimulation need to be suppressed. Yi et al. [40] used a combination of digital filtering and blanking to remove artefacts due to FES from the EMG signal. Keller and Popovic [41] used a stimulation artifact template which was adapted online by averaging successive artifacts and subtracted it from the signal after each stimulation spike. Schauer et al. [42] used a mute input of the amplifier for suppression and reduction of the artefacts during EMG acquisition, and only the EMG signal between the stimulation pulses was processed further. Empirical mode decomposition was used by Pilkar et al. to extract the voluntary EMG during FES, which has the advantage, that no information on the stimulation artefacts is required, but looses high frequency components of the EMG [43].

In contrast to these FES-related studies, the current study focuses on sensory stimulation, i.e., stimulation below the motor threshold. Compared to FES, the application of electrical stimulation for sensory substitution is characterized by more localized current paths, lower intensities, and, most importantly, lack of electrical motor response (M-wave). Hence, electrotactile stimulation has a lower impact on the EMG signal. On the other hand, the requirements to EMG signal quality are more strict in myocontrol than FES, especially when the number of functions to discriminate is large.

Only few previous studies investigated the influence of electrical stimulation artifacts on myoelectrically controlled prostheses. Using neural network classifiers for prosthesis control, Arieta et al. [44] showed that the control performance decreased with the presence of stimulation artifacts, with an effect dependent on the stimulation levels. Almström et al. [37] proposed that a distance between stimulation and EMG pick up sites of at least $60 \mathrm{~mm}$ suffices to avoid significant interference of the stimulation with the prosthesis control. However, this solution is not always feasible in patients with high amputation level, and other 


\section{INCLUDING MYOCONTROL: THE CHALLENGE OF STIMULATION ARTEFACTS}

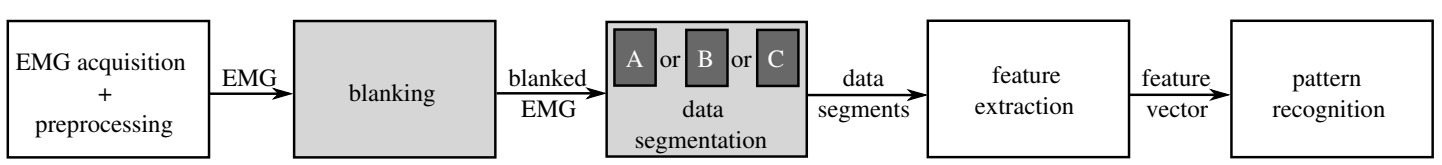

Figure 3.1: Modified signal processing chain with blanking to allow closing the loop in myoelectric control using pattern recognition and electrotactile stimulation. The modifications include zeroing of the samples affected by an artefact (blanking) and three methods (VarA, VarB, and VarC) for segmenting the blanked signals before the feature extraction. For a description of the data segmentation methods, see section 3.2. [35], (c) 2015 IEEE.

approaches to deal with artefacts need to be developed.

\subsection{Artefact blanking in pattern recognition}

The classic signal processing chain for pattern recognition in prosthesis control comprises the following steps [45]: First, the EMG is acquired and pre-processed (e.g., filtered). Afterwards, the acquired data are organized into segments which are then used to compute abstract features. Finally, a pattern recognition approach is applied on the feature vector to obtain the information that is relevant for the control of the prosthetic limb, such as the current motion intended by the amputee.

Again, in a closed-loop prosthetic system with electrocutaneous feedback, the EMG signals in the aforementioned signal processing chain are corrupted with stimulation artefacts. In order to handle such inputs, it is proposed to introduce an additional step into the classic signal processing chain, namely blanking (i.e., zeroing any data samples affected by an artefact), and to modify the segmentation of the data before feature extraction. These proposed changes are highlighted in Fig. 3.1. In principle, an EMG signal with the artefacts blanked could be used in the same way for the feature extraction and subsequent pattern recognition as if it were an artefact-free EMG signal, i.e., blanked samples are treated as regular EMG data and included in the feature calculation. However, it is to be expected that artificially induced zeros in the EMG data negatively affect the outcome of the pattern recognition algorithm, since they were not included in the training set. Alternatively, the zeroed samples can be discarded for the calculation of the features.

In this study, three methods for dealing with blanked signal intervals in the data segmentation were implemented and compared. They shall henceforth be referred to as variants A, B and C. According to variant A (VarA), the blanked samples are retained, so that the data segments for feature calculation include 
the zeroed intervals (Fig. 3.2a). In variant B (VarB), any blanked samples are removed from the signal, so that the samples used for feature extraction are only those not blanked (Fig. 3.2b). Thus, the samples are collected from the same time interval as in VarA, but the resulting data segment has fewer samples (nonblanked samples only), and the sample number can also vary from segment to segment. In variant $\mathrm{C}$ (VarC), the zeroed intervals are discarded as in VarB and only non-blanked samples are used. However, in order to compensate for the discarded samples, the time interval for collecting data is prolonged, i.e., further non-blanked samples are included until the number of samples is the same as in the data segment in VarA. Consequently, in VarC, the pattern recognition result is updated with a larger delay with respect to VarA and VarB due to the extended time interval needed to collect a sufficient number of non-blanked samples.

\subsubsection{Methods}

\subsubsection{Pattern Recognition}

In order to evaluate the feasibility of the proposed approaches, an exemplary test setup for pattern recognition in prosthesis control was selected. As a representative pattern recognition task, the classification of eight motions and grasp types was chosen. Additionally, four different feature sets were extracted from the EMG data in order to determine if the choice of feature space influences the effectiveness of the proposed artefact removal approach. Combined with the three proposed data segmentation variants, this provided a number of scenarios that were tested and compared. Finally, each scenario was investigated with virtual blanking and applied blanking using two experimental data sets.

\subsubsection{Classification Algorithm and Features}

In this study, linear discriminant analysis (LDA) [46-48] was employed. Four widely used time domain features $[46,48-50]$ and three frequency domain features were calculated from the EMG signals. The time domain features were the root mean square value (RMS), zero crossings (ZC), slope sign changes (SSC), and wave length (WL). The combination of LDA with these features has been shown to yield good classification results and to perform similarly or better than other, more complex algorithms (see $[47,48]$ for extensive comparisons). As frequency domain features, the first three cepstral coefficients (3xCEPS) [51] were used. These seven features were arranged into four feature sets that were separately evaluated (Table 3.1). The first and simplest feature set ('RMS') only comprised the RMS values. The second ('RMS+TD') was the classic set of RMS, ZC, SSC and WL. The third set ('RMS+FD') comprised RMS and 3xCEPS, and the final set ('RMS+TD+FD') combined all seven features. For classifier training, 


\section{INCLUDING MYOCONTROL: THE CHALLENGE OF STIMULATION ARTEFACTS}
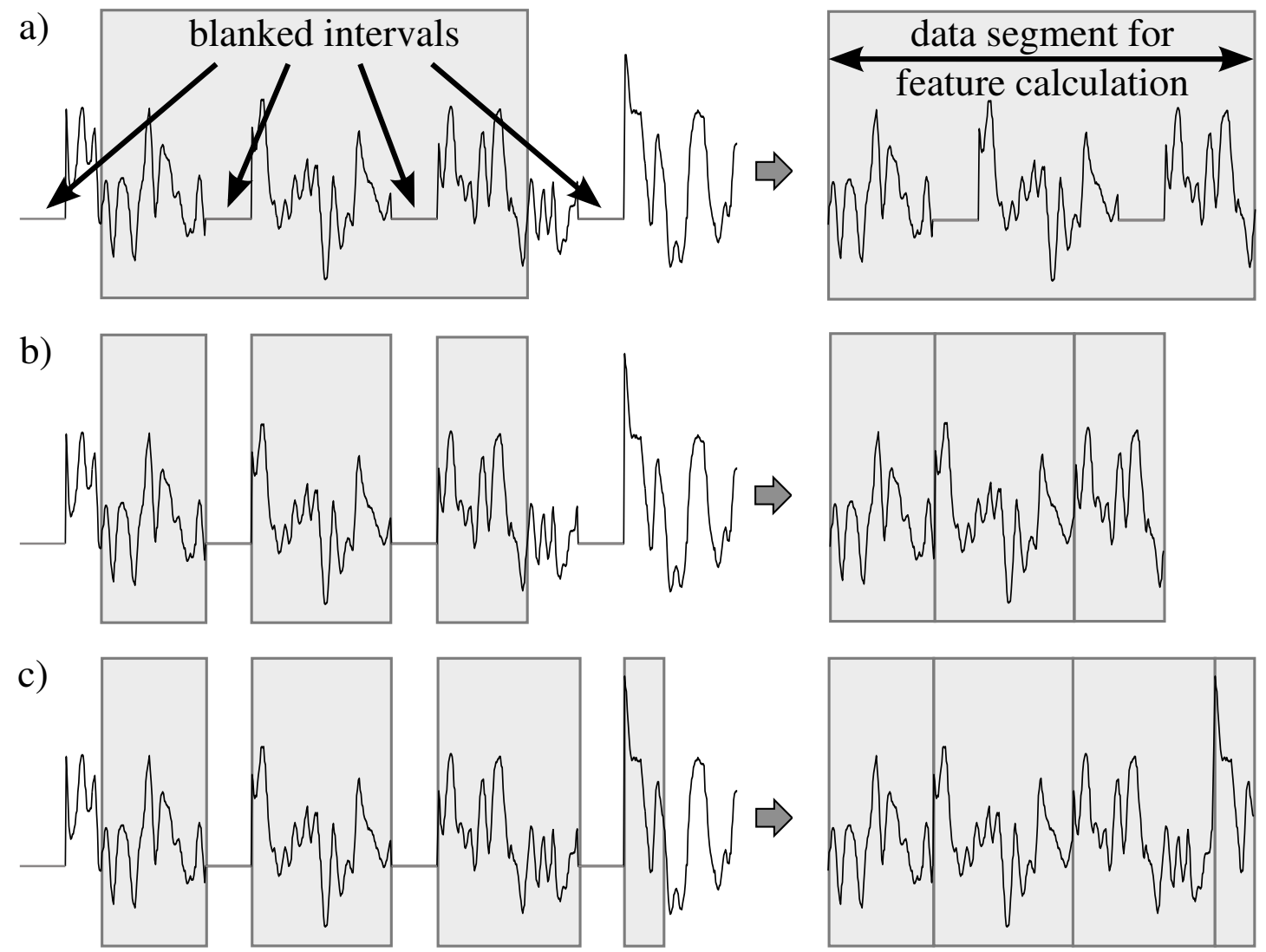

Figure 3.2: Blanking and data segmentation for feature calculation. Left side: segmentation process. Right side: resulting data segments. a) variant A (VarA), artefact blanking with original segmentation; b) variant B (VarB), modified segmentation approach, where blanked data samples are discarded, thus decreasing the number of samples used for the feature calculation; c) variant C (VarC), modified segmentation approach, where blanked samples are discarded and the number of samples for feature calculation is the same as in VarA. [35], (C) 2015 IEEE. 
Table 3.1: Feature Types and Feature Sets

\begin{tabular}{lccccc}
\hline & RMS & ZC & SSC & WL & $3 x C E P S$ \\
\hline 'RMS' & $\mathrm{x}$ & & & & \\
'RMS+TD' & $\mathrm{x}$ & $\mathrm{x}$ & $\mathrm{x}$ & $\mathrm{x}$ & \\
'RMS+FD' & $\mathrm{x}$ & & & & $\mathrm{x}$ \\
'RMS+TD+FD' & $\mathrm{x}$ & $\mathrm{x}$ & $\mathrm{x}$ & $\mathrm{x}$ & $\mathrm{x}$ \\
\hline RMS: root mean square values, ZC: zero crossings, \\
SSC: slope sign changes, WL: wave length, CEPS: \\
cepstral coefficients. [35], (C) 2015 IEEE.
\end{tabular}

the respective features were calculated for each available EMG channel based on data contained in time windows of $128 \mathrm{~ms}$ with $90 \%$ overlap, so that one class decision was obtained every $12.5 \mathrm{~ms}$.

\subsubsection{Virtual and Applied Blanking}

Two data sets were analyzed. First, artefact-free EMG data were blanked to emulate a wide variety of artefact conditions. This approach, hereafter called virtual blanking, allowed great flexibility with respect to the analysis of the influence of the blanking parameters, e.g., duration and frequency. Second, blanking of real stimulation artefacts, hereafter called applied blanking, was implemented for a data set of EMG signals contaminated by actual stimulation artefacts. These stimulation artefacts were recorded under a range of stimulation conditions (cf. section 3.2.1.5 for details on data acquisition).

For the virtual blanking, 39 stimulation frequencies between $10 \mathrm{~Hz}$ and $200 \mathrm{~Hz}$, as well as 30 artefact durations from $0.5 \mathrm{~ms}$ to $5 \mathrm{~ms}$ were simulated. The blanking was implemented by setting recurring sections of artefact-free EMG data to zero in all channels, according to the selected stimulation frequency and artefact duration, as if the artefact was present. In the case of applied blanking, artefacts were created by using stimulation frequencies of up to $150 \mathrm{~Hz}$ and a stimulation pulse width of up to $800 \mu \mathrm{s}$. The artefact positions and duration could be determined from the stimulation signal, and the affected samples were set to zero for all EMG channels.

Although all the analyses for this study were performed off-line with prerecorded data sets, all the proposed methods can be implemented online with minimal hardware requirements. 


\section{INCLUDING MYOCONTROL: THE CHALLENGE OF STIMULATION ARTEFACTS}

\subsubsection{Outcome Measures}

In order to determine the performance of the classifier on either artefact-free, contaminated, or blanked data, the classification rate $r_{\text {class }}$ was used. The classification rate was defined as the percentage of correct motion type estimates achieved for the given data set. Additionally, for the simulated scenarios, the performance quotient $q$ was defined to evaluate the capability of the proposed blanking approaches to recover the performance of the pattern recognition algorithm with respect to the benchmark condition of artefact-free data. The performance quotient was calculated as

$$
q=\frac{r_{\text {class }}}{r_{\text {class }, \text { ctrl }}},
$$

where $r_{\text {class,ctrl }}$ is the benchmark performance for an artefact-free data set, and $r_{\text {class }}$ is the classification rate achieved for the same data set after virtual artefact blanking was applied.

Further measures employed in the evaluation of the virtual blanking were the portion $\rho_{0.95}$ of the investigated stimulation frequency $\times$ artefact duration domain for which $q>0.95$ could be achieved, as well as the intersections of the $q>0.95$ condition with the investigated domain borders. For the intersections, $d_{\text {art,max }}$ was the maximum artefact duration for which $q>0.95$ could still be achieved, when the highest stimulation frequency of $f_{\text {stim }}=200 \mathrm{~Hz}$ was assumed, while $f_{\text {stim,max }}$ was the maximum frequency for which $q>0.95$ still held, if the longest artefact duration of $d_{\text {art }}=5 \mathrm{~ms}$ was used. These measures were also used for inter-approach comparisons.

For statistical comparisons, t-tests, one-way and three-way repeated measures ANOVA and Newman-Keuls post-hoc tests were applied. The within subject factor for the one-way ANOVAs was the active stimulation ration $\kappa$. Within subject factors for the three-way ANOVA were the blanking method, the feature set, and the active stimulation ratio $\kappa$. The latter was defined as the product of the stimulation frequency $f_{\text {stim }}$ and the stimulation pulse width $p w_{\text {pulse: }}$ :

$$
\kappa=p w_{\text {pulse }} \cdot f_{\text {stim }} .
$$

Both $f_{\text {stim }}$ and $p w_{\text {stim }}$ have an analogous effect on the EMG quality. A longer pulse width increases the artefact duration while a higher frequency introduces more artefacts per second, thereby leading to a larger number of corrupted samples. The active stimulation ratio $\kappa$ was adopted to conveniently describe this common effect.

\subsubsection{Experimental Setup and Protocol}

To collect the input data for this study, six bipolar EMG channels were recorded using an analogue EMG amplifier (AnEMG12, OTBioelettronica, Italy). Each 

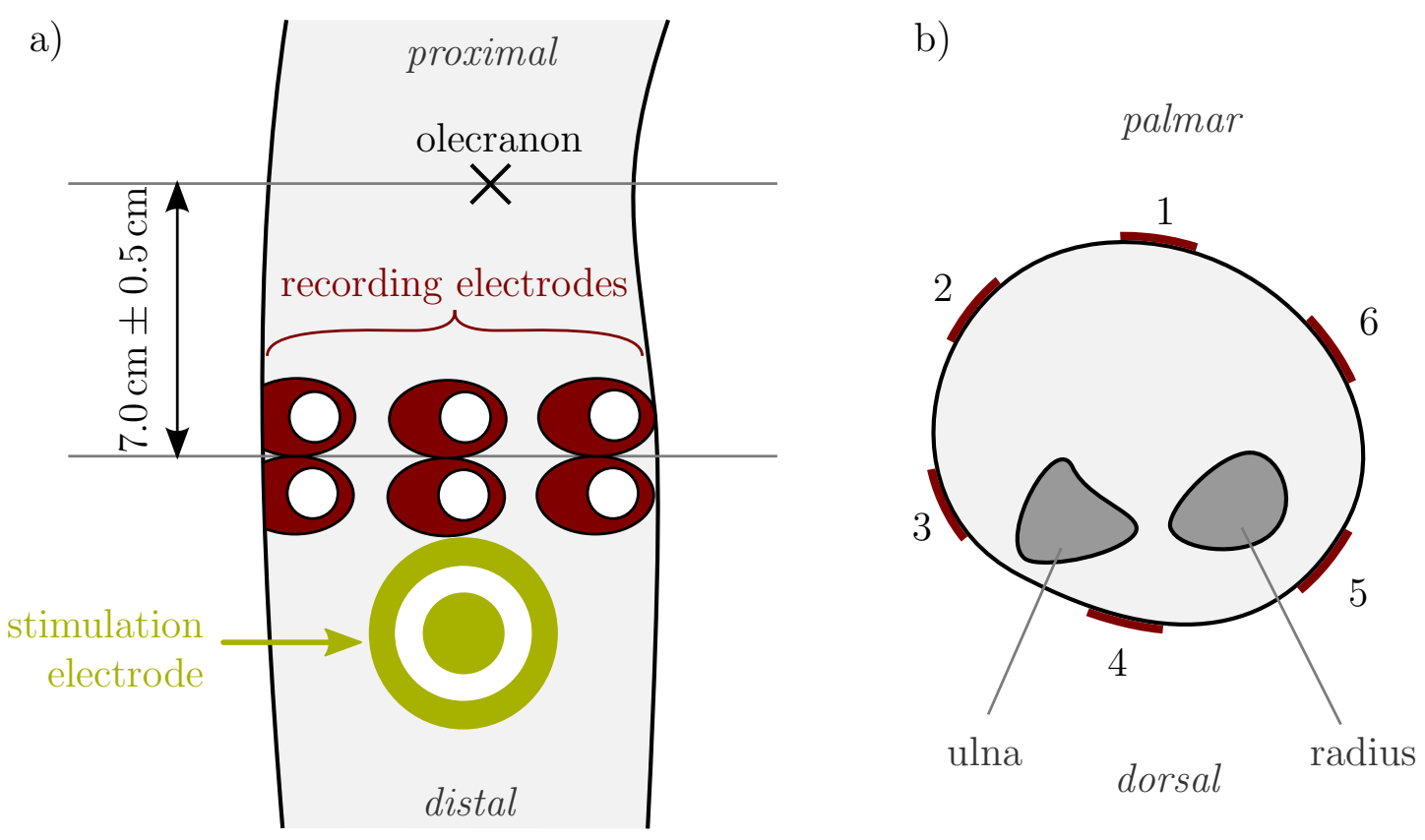

Figure 3.3: Placement of stimulation and recording electrodes: a) Dorsal view of the forearm with recording and stimulation electrodes. b) Transversal view of the forearm depicting recording electrode distribution. Note that the stimulation electrode was placed directly next to the recording electrodes in order to emulate the lack of skin surface for electrode placement on a residual limb. Adapted from [35], (c) 2015 IEEE. 


\section{INCLUDING MYOCONTROL: THE CHALLENGE OF STIMULATION ARTEFACTS}
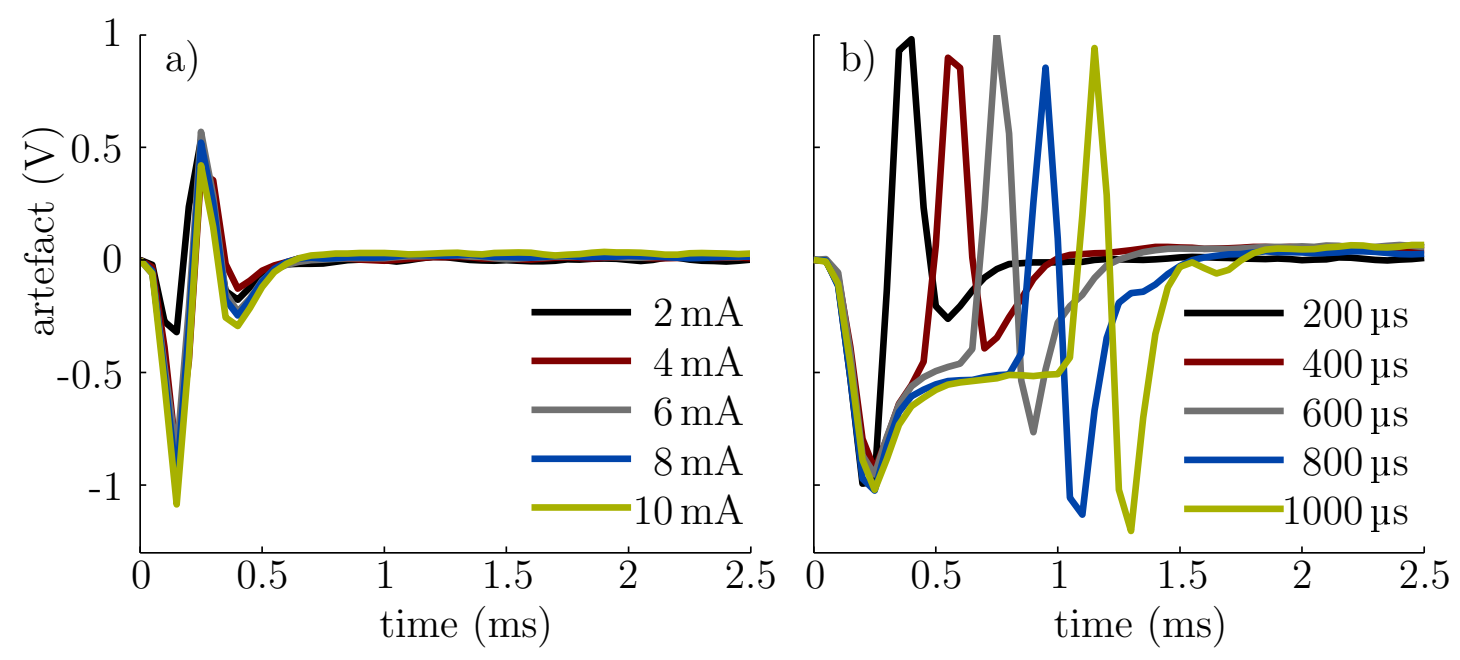

Figure 3.4: Stimulation artefact shape, averaged over 50 artefacts; a) constant pulse width of $50 \mu \mathrm{s}$ and varying peak current; b) constant peak current of $1 \mathrm{~mA}$ and varying pulse width. Signals were low-pass filtered $(4.4 \mathrm{kHz}$ cut-off frequency), amplification gain was 1000 and sampling frequency $20 \mathrm{kHz}$. Note, that stimulation current determines the peak to peak value, while the pulse width affects both the peak to peak value and the artefact duration. Adapted from [35], (c) 2015 IEEE.

channel was equipped with a band-pass filter (cut-off frequencies $10 \mathrm{~Hz}$ and $900 \mathrm{~Hz}$ ) and adjustable gain. Disposable pre-gelled self-adhesive $\mathrm{Ag} / \mathrm{AgCl}$ recording electrodes (Ambu Neuroline 720, Ambu, Denmark) were placed equidistantly and circumferentially around the forearm, $7.5 \pm 0.5 \mathrm{~cm}$ distal from the olecranon (Fig. 3.3b). For stimulation, one disposable concentric self-adhesive surface electrode (CODE501500, Ø $42 \mathrm{~mm}$, Spes Medica, Italy) was placed distal with respect to the dorsal recording electrode pair (Fig. 3.3a). The electrode was placed directly next to the recording sites to emulate the lack of space that can be expected in practical applications. The inner electrode was the cathode (stimulation point), the outer ring was the anode. Stimulation was biphasic with a rectangular pulse to depolarize the cutaneous afferents and a subsequent exponential waveform of opposite polarity for charge equalization of the tissue. The stimulation was generated by an externally controlled isolated bipolar constant current stimulator (DS5, Digitimer, United Kingdom).

Preliminary experiments indicated that the shape of the observed stimulation artefact in the EMG signal largely depends on the stimulation parameters, as well as on the preprocessing of the signal. Fig. 3.4 shows examples of artefact shapes for different stimulation pulse widths and currents. It can be observed that the current amplitude determines the peak-to-peak value of the artefact 
waveform, whereas the pulse width also influences its duration. The artefact peak-to-peak value does not influence a blanking approach while the artefact duration is of importance, since it determines the number of samples that need to be zeroed. Hence, as this study focuses on the evaluation of blanking and not on the actual sensation that is elicited by the stimulation, the current amplitude was set to a constant low value $\left(I_{\text {pulse }}=1 \mathrm{~mA}\right)$. The parameters important for blanking, namely the pulse width and stimulation frequency, were selected according to two criteria: 1) the need to cover the range of interest for the actual prosthetic applications $[19,20]$, and 2) to create data sets with different amounts of corrupted data.

Altogether, nine sets of stimulation parameters $\left[f_{\text {stim }}, p w_{\text {pulse }}\right]$ were chosen, where $f_{\text {stim }} \in\{50 \mathrm{~Hz}, 100 \mathrm{~Hz}, 150 \mathrm{~Hz}\}$ and $p w_{\text {pulse }} \in\{200 \mu \mathrm{s}, 400 \mu \mathrm{s}, 800 \mu \mathrm{s}\}$. These stimulation settings corresponded to values of the active stimulation ratio $\kappa$ ranging from $1 \%$ to $12 \%$. With these sets, the elicited sensation was below the pain threshold and no saturation of the EMG amplifier occurred. With the setup used in this study, the amplifier did not saturate even when the stimulation current was increased substantially, up to the level where it started becoming uncomfortable or painful (e.g., up to $15 \mathrm{~mA}$ at $150 \mu \mathrm{s}$ and up to $4 \mathrm{~mA}$ at $1000 \mu \mathrm{s}$ ).

Seven able-bodied subjects (three women, four men; age $27 \pm 3$ years) volunteered to participate in the recordings. All subjects signed a consent form approved by the local ethics committee. Subjects were comfortably seated, with their arm and hand positioned relaxed next to the side of the trunk. The concept of electrocutaneous stimulation was explained to the subjects, and a low-intensity stimulation was applied to their right forearm, so that they could get accustomed to the sensation.

The subjects were asked to repeat with their right hand a motion or grasp type shown to them on a computer screen and maintain it for two seconds, while they received electrocutaneous stimulation on the same arm. The motions and grasps were: i) flexion and ii) extension of the wrist, iii) opening and iv) closing of the hand, v) pronation and vi) supination, vii) pinch grip, and viii) hand relaxed. During each $2 \mathrm{~s}$ trial dedicated to a single, specified grasp type or motion, subjects were asked to keep the posture at a self-chosen, comfortable contraction level.

For each stimulation condition, five trials for each grasp type and motion were recorded. Additionally, 15 trials for each grasp type and motion were recorded without applying electrical stimulation for purposes of classifier training (10 trials), as well as performance benchmarking (classification of artefact-free data) and virtual blanking ( 5 trials). Trials were arranged randomly with respect to the order of the motions, as well as to the stimulation conditions. The total duration of the recording session, including informing and preparing the participant, was approximately $90 \mathrm{~min}$. 


\section{INCLUDING MYOCONTROL: THE CHALLENGE OF STIMULATION ARTEFACTS}

For six of the subjects, data sets for each of these conditions were recorded, whereas for one subject, a reduced series comprising only six parameter sets was collected. Conditions with pulse width $p w_{\text {pulse }}=800 \mu$ s were omitted on the subject's request since the subject felt uncomfortable with this stimulation setting.

\subsubsection{Results}

\subsubsection{Impact of Stimulation Artefacts}

With the setup and stimulation parameters used in this study, the electrical stimulation artefacts occurring on the EMG channels closest to the stimulation electrode were at least of the same magnitude as the EMG signal amplitude. Artefact durations ranged from approximately $0.9 \mathrm{~ms}$ to $2.7 \mathrm{~ms}$. This means that at the highest stimulation frequency up to approximately $40 \%$ of the samples were contaminated by artefacts. Fig. 3.5 shows representative data recorded during voluntary flexion of the wrist, illustrating artefact-free EMG and an equivalent signal contaminated with stimulation artefacts. Stimulation neither elicited motor responses (no M-wave) nor saturated the amplifier. With increasing distance of the EMG pickup electrodes to the stimulation electrode, the impact of the stimulation on the EMG signal gradually diminished. This can be seen in Fig. 3.5c, which shows a six channel recording from the relaxed forearm (no voluntary muscle contraction). The magnitude of the artefact was largest for channel 4 and neighboring channels (for electrode placement, cf. Fig. 3.3), and lowest for channel 1 and neighboring channels. The shape of the artefact also changed from a monopolar waveform (channel 2) to waveforms with two (channel 6) or three (channel 4) phases. However, the artefact duration was approximately the same for all channels.

The presence of artefacts had a strong detrimental effect on the classification rate $r_{\text {class }}$, as compared to the classification of the artefact-free control data sets. For controls, the following overall classification rates $r_{\text {class,ctrl }}$ (mean \pm standard deviation) were achieved across subjects: $84.1 \% \pm 7.0 \%$ when using the feature set 'RMS', $93.5 \% \pm 5.6 \%$ for 'RMS+TD', $93.0 \% \pm 5.7 \%$ for 'RMS+FD', and $94.8 \% \pm 5.0 \%$ for 'RMS+TD+FD'. When attempting to classify the contaminated data sets without removing the artefacts, $r_{\text {class }}$ across subjects and stimulation conditions decreased to average values of $41.4 \pm 28.8 \%, 38.8 \pm 24.5 \%$, $27.1 \pm 15.7 \%$ and $34.9 \pm 24.4 \%$, respectively. The drop in performance was statistically significant in all cases $(p<0.001)$.

\subsubsection{Virtual Blanking}

Figure 3.6 shows the decrease in performance quotient $q$ when increasing stimulation frequency and/or artefact duration for all feature sets averaged over all 

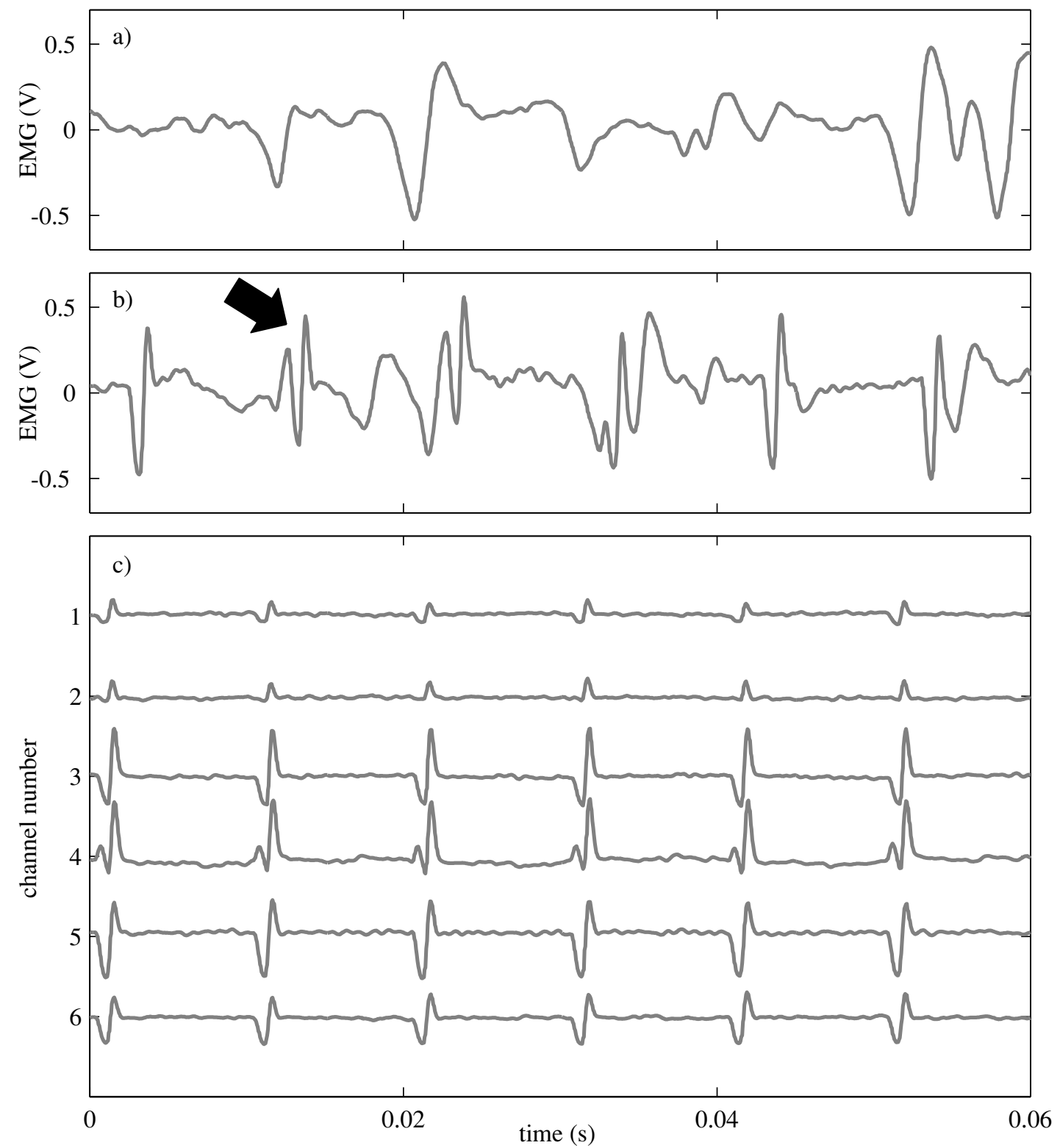

Figure 3.5: Influence of electrical stimulation: a) artefact-free EMG signal; b) EMG signal contaminated by electrical stimulation artefacts; c) attenuation of artefacts along the EMG channels, stimulation site next to channel 4 (cf. Fig. 3.3 for channel numbering and electrode placement). Stimulation properties for b) and c): biphasic stimulation, pulse width $800 \mu \mathrm{s}$, amplitude $1 \mathrm{~mA}$, stimulation frequency $100 \mathrm{~Hz}$. Note that shape and size of the stimulation artefacts introduced into the recorded EMG signal depend on the relative placement of the recording and stimulation electrodes. [35] (C) 2015 IEEE. 


\section{INCLUDING MYOCONTROL: THE CHALLENGE OF STIMULATION ARTEFACTS}

Table 3.2: $\rho_{0.95}$, portion of the investigated frequency/duration domain where $q>0.95$ is achieved (mean \pm standard deviation) [35] (C) 2015 IEEE.

\begin{tabular}{lccc}
\hline & VarA & VarB & VarC \\
\hline RMS & $44.1 \% \pm 11.9 \%$ & $96.3 \% \pm 3.5 \%$ & $99.7 \% \pm 0.1 \%$ \\
RMS+TD & $34.7 \% \pm 9.8 \%$ & $73.3 \% \pm 7.5 \%$ & $76.5 \% \pm 7.3 \%$ \\
RMS+FD & $38.2 \% \pm 31.6 \%$ & $53.2 \% \pm 24.7 \%$ & $57.3 \% \pm 26.1 \%$ \\
RMS+TD+FD & $37.0 \% \pm 14.9 \%$ & $67.7 \% \pm 16.4 \%$ & $72.1 \% \pm 11.9 \%$ \\
\hline
\end{tabular}

Table 3.3: $f_{\text {stim,max }}$, Intersection with Investigated Artefact Duration Domain Border for which $q>0.95$ is still achieved (mean \pm standard deviation) [35] (c) 2015 IEEE.

\begin{tabular}{lccc}
\hline & VarA & VarB & VarC \\
\hline RMS & $21 \mathrm{~Hz} \pm 9 \mathrm{~Hz}$ & $111 \mathrm{~Hz} \pm 40 \mathrm{~Hz}$ & $184 \mathrm{~Hz} \pm 2 \mathrm{~Hz}$ \\
RMS+TD & $20 \mathrm{~Hz} \pm 7 \mathrm{~Hz}$ & $74 \mathrm{~Hz} \pm 7 \mathrm{~Hz}$ & $84 \mathrm{~Hz} \pm 12 \mathrm{~Hz}$ \\
RMS+FD & $34 \mathrm{~Hz} \pm 21 \mathrm{~Hz}$ & $34 \mathrm{~Hz} \pm 13 \mathrm{~Hz}$ & $50 \mathrm{~Hz} \pm 25 \mathrm{~Hz}$ \\
RMS+TD+FD & $26 \mathrm{~Hz} \pm 11 \mathrm{~Hz}$ & $63 \mathrm{~Hz} \pm 23 \mathrm{~Hz}$ & $74 \mathrm{~Hz} \pm 18 \mathrm{~Hz}$ \\
\hline
\end{tabular}

subjects. The gray areas in the horizontal planes of the 3D plots indicate $\rho_{0.95}$. The results for all performance measures are summarized in Tables 3.2-3.4. The simulations using virtual blanking showed that for the 'RMS' feature set (Fig. $3.6 \mathrm{a}-\mathrm{c})$ the approach VarA yielded good results $(q>0.95)$ only if either low frequencies $\left(f_{\text {stim }} \leq 21.43 \mathrm{~Hz}\right)$ or low artefact durations $\left(d_{\text {art }} \leq 0.36 \mathrm{~ms}\right)$ were considered. When using the approaches $\operatorname{VarB}$ and $\operatorname{VarC}$, even for high values of both interference factors, the performance quotient was close to 1 . This was also reflected in high average $\rho_{0.95}$ values of $96.3 \% \pm 3.5 \%$ and $99.7 \% \pm 0.1 \%$,

Table 3.4: $d_{\mathrm{art}, \max }$, Intersection with Investigated Frequency Domain Border for which $q>0.95$ is still achieved (mean \pm standard deviation) [35] (C) 2015 IEEE.

\begin{tabular}{lccc}
\hline & VarA & VarB & VarC \\
\hline RMS & $0.4 \mathrm{~ms} \pm 0.5 \mathrm{~ms}$ & $3.7 \mathrm{~ms} \pm 0.7 \mathrm{~ms}$ & $4.5 \mathrm{~ms} \pm 0.2 \mathrm{~ms}$ \\
RMS+TD & $0.1 \mathrm{~ms} \pm 0.2 \mathrm{~ms}$ & $1.1 \mathrm{~ms} \pm 0.3 \mathrm{~ms}$ & $1.2 \mathrm{~ms} \pm 0.3 \mathrm{~ms}$ \\
RMS+FD & $0.4 \mathrm{~ms} \pm 0.7 \mathrm{~ms}$ & $0.9 \mathrm{~ms} \pm 0.7 \mathrm{~ms}$ & $0.9 \mathrm{~ms} \pm 0.7 \mathrm{~ms}$ \\
RMS+TD+FD & $0.2 \mathrm{~ms} \pm 0.5 \mathrm{~ms}$ & $1.0 \mathrm{~ms} \pm 0.7 \mathrm{~ms}$ & $1.2 \mathrm{~ms} \pm 0.5 \mathrm{~ms}$ \\
\hline
\end{tabular}




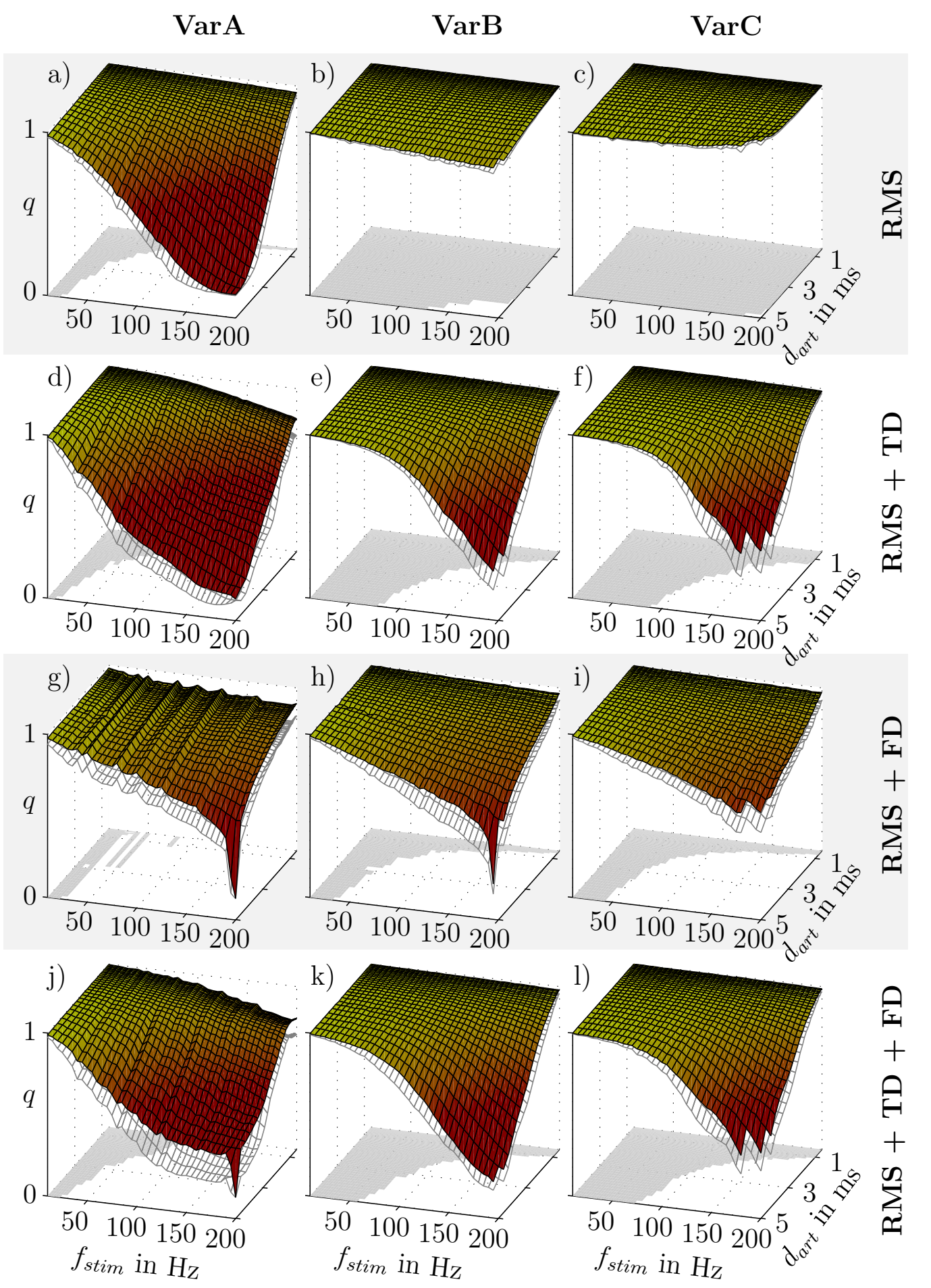

Figure 3.6: Performance quotient $q$ as a function of artefact duration $d_{\text {art }}$ and stimulation frequency $f_{\text {stim }}$, shown for all investigated feature sets and segmentation approaches. Coloured surface shows mean value $\mu$, grey grid indicates subtraction of standard deviation $\sigma: \mu-\sigma$. Grey areas indicates $\rho_{0.95}$ (fraction of the $f_{\text {stim }} \times d_{\text {art }}$ domain for which $\left.q>0.95\right)$. Adapted from [35], (C) 2015 IEEE. 


\section{INCLUDING MYOCONTROL: THE CHALLENGE OF STIMULATION ARTEFACTS}

respectively. In comparison, for approach VarA, the drop in the performance quotient $q$ for the simultaneous increase in artefact duration and stimulation frequency manifested in an average $\rho_{0.95}$ of $44.1 \% \pm 11.9 \%$. This was less than half of what was obtained for variants $\operatorname{VarB}$ and $\operatorname{VarC}$, and thus significantly lower $(p<0.001)$.

In the case of the feature sets 'RMS+TD' and 'RMS+TD+FD' (Fig. 3.6 d-f and $j-1$ ), the simulation results for the approach VarA were comparable to the results for the feature set 'RMS'. Similarly, the approaches VarB and VarC were significantly better than $\operatorname{VarA}(p<0.01)$ in all the outcome measures. However, the quotient $q$ for $\operatorname{VarB}$ and $\operatorname{VarC}$ was not as consistently high over the whole artefact duration $\times$ stimulation frequency domain as it was in the case of the 'RMS' feature set. Instead, it gradually dropped when both frequency and duration increased, resulting in a $\rho_{0.95}$ value that was considerably smaller than for the 'RMS' feature set $(p<0.01)$.

For the 'RMS+FD' feature set, the performance quotient was already adversely affected for lower values of the disruptive factors. This resulted in comparatively small values of $\rho_{0.95}$ and $f_{\text {stim,max }}$ for all three methods (Tables 3.2 and 3.3, Fig. $3.6 \mathrm{~g}-\mathrm{i})$. However, for higher values of $f_{\text {stim }}$ and $d_{\text {art }}$ the rate of decrease of $q$ was more moderate, so that the performance quotient in the more challenging region (high values of both $f_{\text {stim }}$ and $d_{\text {art }}$ ) of the artefact duration $\times$ stimulation frequency plane was higher than for the other feature sets (with the exception of 'RMS' combined with approach VarB or VarC, see above).

In summary, for each of the investigated feature sets, $\rho_{0.95}$ was always smallest for the approach VarA, and greatest for the variant VarC. The overall highest value for $\rho_{0.95}$ was achieved for 'RMS' and methods VarB and VarC. Similarly, the average maximum tolerable artefact duration $d_{\text {art,max }}$ was generally lowest for the approach VarA, whereas the highest value was predicted for the feature set 'RMS' and variant VarC (Table 3.4). The same held for the average maximum tolerable stimulation frequency $f_{\text {stim,max }}$ (Table 3.3 ).

Finally, as is summarized in Table 3.5, for the feature sets 'RMS' and 'RMS+TD', the approaches VarB and VarC showed to be significantly superior to VarA with respect to all outcome measures. A statistically significant difference in the performance of $\operatorname{VarB}$ and $\operatorname{VarC}$ was obtained for the value of $\rho_{0.95}$, as using the approach VarC resulted in significantly higher values for this performance measure than using VarB. For the feature set 'RMS+TD+FD', a significant difference between VarA and VarC was found, with the latter approach resulting in significantly higher mean values for all outcome measures. Only for the feature set 'RMS+FD', there was no statistically significant difference in the performance of the three approaches. 
Table 3.5: Statistically Significant Differences $(p<0.001)$ within each Feature Set for Results Given in Tables 3.2-3.4. [35] (C) 2015 IEEE.

\begin{tabular}{lccc}
\hline & $\operatorname{Var} \mathrm{A}$ & $\operatorname{VarB}$ & $\operatorname{VarC}$ \\
\hline RMS & $\mathrm{B}, \mathrm{C}$ & $\mathrm{A}, \mathrm{C}^{\rho}$ & $\mathrm{A}, \mathrm{B}^{\rho}$ \\
RMS+TD & $\mathrm{B}, \mathrm{C}$ & $\mathrm{A}, \mathrm{C}^{\rho}$ & $\mathrm{A}, \mathrm{B}^{\rho}$ \\
RMS+FD & & & \\
RMS+TD+FD & $\mathrm{C}$ & & $\mathrm{A}$ \\
\hline
\end{tabular}

Letters indicate, that the respective approach (column header) significantly differs from the approaches corresponding to the letter. Index $\rho$ indicates differences valid only for the results in Table 3.2 .

\subsubsection{Applied Blanking}

Fig. 3.7 shows the average classification rates $r_{\text {class }}$ that could be achieved for contaminated and blanked data depending on the active stimulation ratio $\kappa$ for each of the investigated methods and feature sets. The three-way interaction between the blanking method, stimulation ratio and feature set factors was statistically significant $(p<0.001)$. If blanking was not used, the performance decreased dramatically with the increase of the active stimulation ratio $\kappa$, dropping almost exponentially and reaching a plateau at very low levels, often below $20 \%$. Already for $\kappa=1 \%$ the performance drop was statistically significant $(p<0.05)$ with respect to the benchmark level (artefact-free data classification) for all feature sets, except for 'RMS'. For 'RMS', the classification rate for $\kappa=1 \%$ was lower than, but not significantly different from, the benchmark performance. The largest decrease in $r_{\text {class }}$ was observed for the feature set 'RMS+FD'. Here, the presence of artefacts caused a drop of $r_{\text {class }}$ from $93.0 \% \pm 5.7 \%$ to $38.7 \% \pm 17.4 \%$ already for $\kappa=1 \%$.

For all stimulation scenarios and each of the feature sets except 'RMS', the classification performance was significantly improved by using any of the investigated methods $(p<0.001)$. For the feature set 'RMS' and $\kappa=1 \%$, the improvement was not statistically significant, and for 'RMS' and $\kappa=3 \%$ only the methods VarB and VarC yielded significant improvements. Applying variants VarB and VarC yielded significantly better results than VarA for $\kappa \geq 6 \%$ in the case of 'RMS+TD' and 'RMS+TD+FD'. Furthermore, there was a trend indicating that variants VarB and VarC are superior to VarA even for lower values of $\kappa$ and also 


\section{INCLUDING MYOCONTROL: THE CHALLENGE OF STIMULATION ARTEFACTS}

Table 3.6: Recovery to Benchmark Performance Depending on Feature Set and stimulation ratio $\kappa$. [35] (C) 2015 IEEE.

\begin{tabular}{ccccc}
\hline$\downarrow \kappa$ & RMS & RMS+TD & RMS+FD & RMS+TD+FD \\
\hline $1 \%$ & A, B, C & A, B, C & A, B, C & A, B, C \\
$2 \%$ & A, B, C & A, B, C & B, C & A, B, C \\
$3 \%$ & A, B, C & B, C & B, C & B, C \\
$4 \%$ & A, B, C & B, C & & \\
$6 \%$ & B, C & & & \\
\hline
\end{tabular}

Letters refer to approaches that were able to recover the benchmark performance. Recovery was deemed to be achieved if the performance was not significantly different from the benchmark performance $(p>0.05)$.

for the feature sets 'RMS' and 'RMS+FD', although in these cases the differences were not statistically significant. There were no significant differences in the performance between methods VarB and VarC. Importantly, all approaches could recover the performance to the level of the benchmark for lower values of $\kappa$, as is indicated in Table 3.6. Again, the methods VarB and VarC were more successful than the approach VarA, i.e., there were many cases where the approaches VarB and VarC could recover the performance to the level of the benchmark, where VarA could not (Table 3.6).

\subsection{Artefact blanking for proportional control}

In contrast to pattern-recognition-based control approaches, where EMG data collected from several channels is analysed to determine the user's intention, in direct proportional control, each motion primitive of a prosthesis is directly controlled by a single EMG channel. For example, a simple hand prosthesis, which can open and close to grasp objects would be controlled by two EMG signals. In transradial amputees, those are usually collected from the wrist extensor and flexor muscles, two muscle groups located in the proximal region of the forearm. The average activity of the signals over a certain time is determined and proportionally mapped to the speed of the motion the prosthesis executes. For instance, a strong contraction of the flexor muscle would cause the prosthesis to close the hand at a high speed.

The signal processing chain is comparable to that described for pattern recognition in section 3.2. As described earlier, EMG data is recorded, subjected to basic 

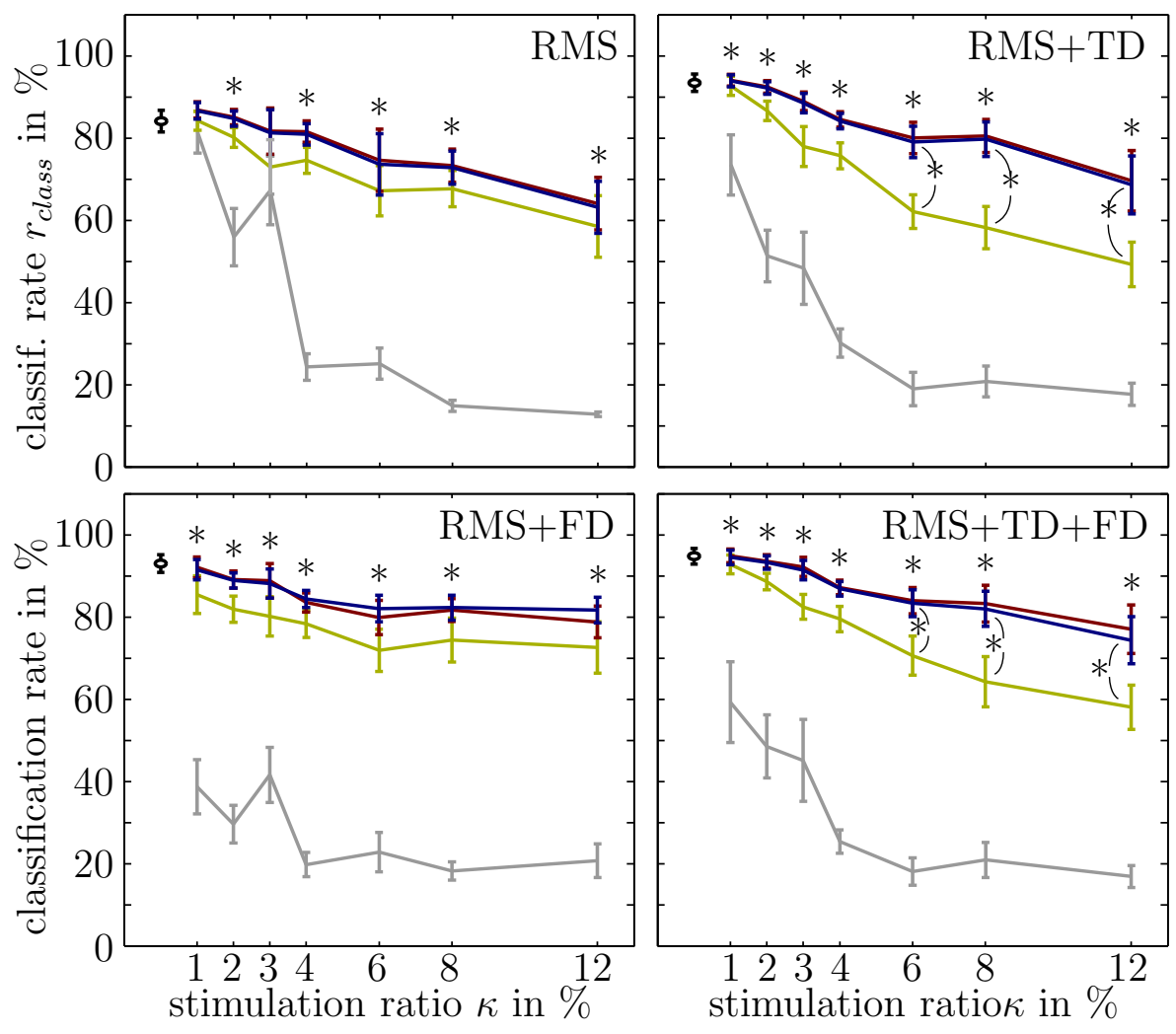

- artifact-free control contaminated

Figure 3.7: Decay of the method performance $r_{\text {class }}$ with rising values of $\kappa$ depending on the blanking approach (mean \pm standard error). Statistically significant performance improvements $(p<0.001)$ as compared to the contaminated data are indicated by an asterisk $\left(^{*}\right)$ above the graph lines. Additionally, if variants VarB and VarC perform significantly better than variant VarA, this is indicated by an asterisk $(*)$ between the graph lines. The performance drops abruptly for the corrupted EMG and it is recovered to a significantly higher level by applying any of the methods. Methods VarB and VarC perform similarly and they appear to be somewhat superior to VarA. Adapted from [35], (C) 2015 IEEE. 


\section{INCLUDING MYOCONTROL: THE CHALLENGE OF STIMULATION ARTEFACTS}

preprocessing, and subsequently organised into segments. Instead of extracting features from the EMG, which are then used as input for a pattern recognition approach, for direct proportional control merely an estimate of the EMG activity within the current time frame needs to be determined. When there is significant activity on two EMG channels controlling different directions of the same degree of freedom of a prosthesis, a comparison of signal strengths is usually made, and the channel showing higher activity wins and its full activity is taken into account for prosthesis control. As a consequence, even if a prosthesis user involuntarily co-activates muscle groups controlling rivalling motion primitives (e.g. opening and closing of the hand), this approach ensures that the motion primitive most likely intended by the user is activated.

In a closed-loop prosthetic system with electrocutaneous feedback, the presence of stimulation artefacts on the measured EMG signal would result in an offset on the control signal. The offset's level would depend on the stimulation frequency, as well as on the artefact shape. It would result in unwanted activation of the prosthesis, sticking of the prosthesis in one of the end positions, or unintentionally fast movements.

Based on the promising results obtained for pattern recognition (cf. section 3.4), it is hypothesised that also for direct proportional control, the detrimental effect of the stimulation can be eliminated by blanking the artefacts from the signal and limiting estimation of EMG activity to those data points carrying undistorted information. In particular, it is suggested to use approach VarB. This method was shown to be successful in restoring the performance of multidimensional EMG pattern recognition back to the baseline for a wide range of stimulation settings without introducing additional delays into the control. Furthermore, in order to facilitate an efficient and low-cost implementation of the procedure, it is proposed to additionally preserve the segment size, i.e., number of samples, used to determine EMG activity and substitute the rejected data samples with a surrogate signal. Hence, the implementation of a first in first out buffer to store data patches is proposed. In the event of an artefact, data stored in the buffer should be used to fill the gap in the signal. To ensure that most recent changes in the signal are taken into account as far as possible, data samples should be read from the buffer in a last in first out manner. This results in a mirrored representation of the EMG signal preceding the artefact. This approach is not different from the approach suggested for pattern recognition, with respect to the original information being lost in both cases. However, it provides a more straightforward online implementation, as it prevents dynamically changing segment sizes. It was assumed that due to the relative robustness of the classic proportional control approach, the inclusion of mirrored signal segments in the activity determination algorithms should not significantly diminish the reliability of the estimate. 


\subsubsection{Methods}

\subsubsection{Proportional control}

In order to test these hypotheses, two-channel proportional control approach commonly implemented in commercial devices was chosen. Here the time window analysed to get an estimate of the current EMG activity of each channel has a length of $128 \mathrm{~ms}$. The root mean square value $R M S$ of the $n$ data samples in that window is used as a measure for EMG activity. It calculates as

$$
R M S(x)=\sqrt{\sum_{n} x_{n}^{2}} .
$$

In order to reduce delays, time windows overlapped by $78 \mathrm{~ms}$, resulting in an effective $20 \mathrm{~Hz}$ update rate for the activity estimate. EMG data was sampled at $1 \mathrm{kHz}$.

\subsubsection{Experimental setup and protocol}

The impact of electrocutaneous stimulation on classic proportional prosthesis control and the applicability of the proposed approach to process contaminated EMG data were evaluated in a practical experiment where subjects performed a grasp-, lift-, and release- related task. The task employed is known as the so-called box and blocks test. This task requires the subjects to grab wooden blocks from one compartment of a box, lift them across a barrier, and subsequently deposit them in a second compartment of the box.

A total number of 15 able-bodied volunteers participated in this study after signing informed consent. The subjects used a commercially available prosthetic hand (Michelangelo hand prosthesis, Otto Bock HealthCare GmbH, Germany) to perform the task. The hand was mounted to the subject's left forearm using a special socket as shown in figure 3.8. Two bipolar EMG electrodes $(13 \mathrm{E} 200=50 \mathrm{AC}$, Otto Bock HealthCare GmbH, Germany) were positioned over the wrist flexor and extensor muscles of that same forearm. By contracting these muscles, subjects were able to close and open the prosthetic hand. A single concentric stimulation electrode was placed on the dorsal side of the forearm with a distance of $1.5 \mathrm{~cm}$ to $2.5 \mathrm{~cm}$ from the EMG recording site. Placement of electrodes is shown in figure 3.9 .

Each participant performed the task under three different conditions. A control condition was designed in order to establish a benchmark measurement of the task performance without the potentially detrimental influence of electrocuteneous stimulation on the EMG control signal. During this control condition, no electrical stimulation was applied. 


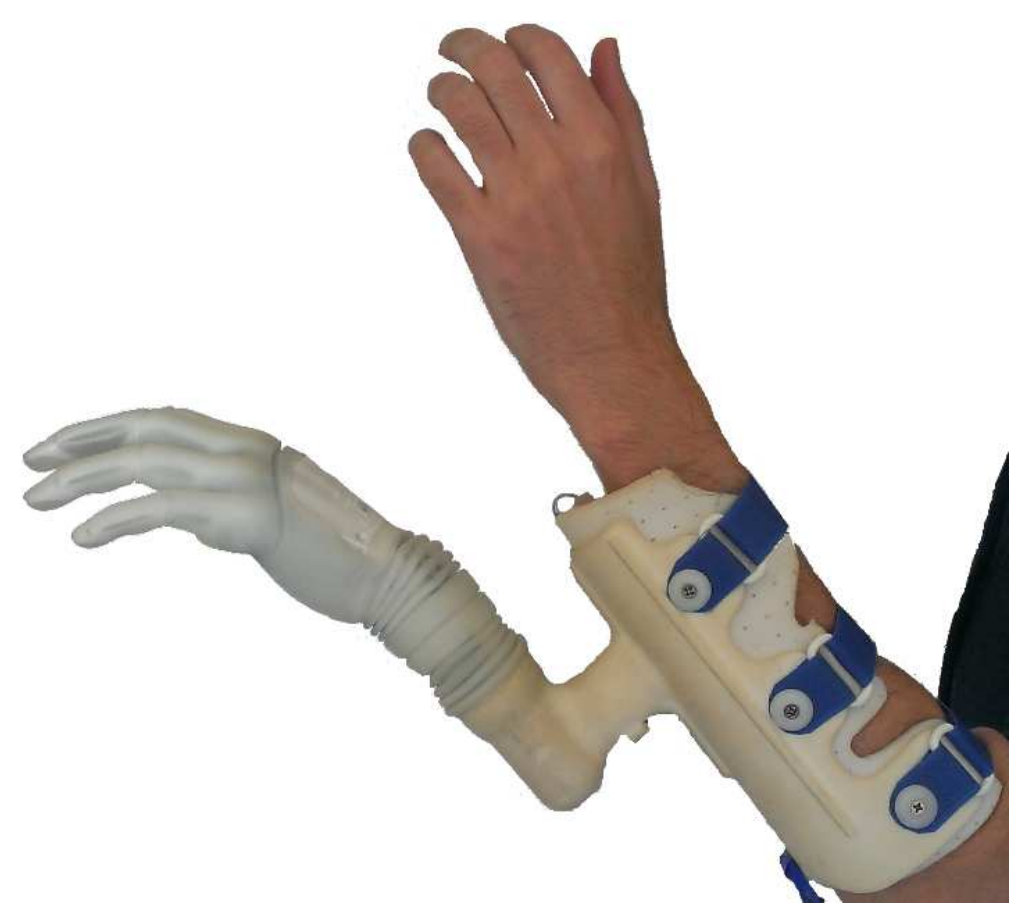

Figure 3.8: The Michelangelo hand prosthesis (Otto Bock HealthCare GmbH, Germany) and special socket for usage with able-bodied subjects. Both stimulation electrode and EMG recording electrodes were placed within the socket. Photo curtsey of Sebastian Amsuess. 


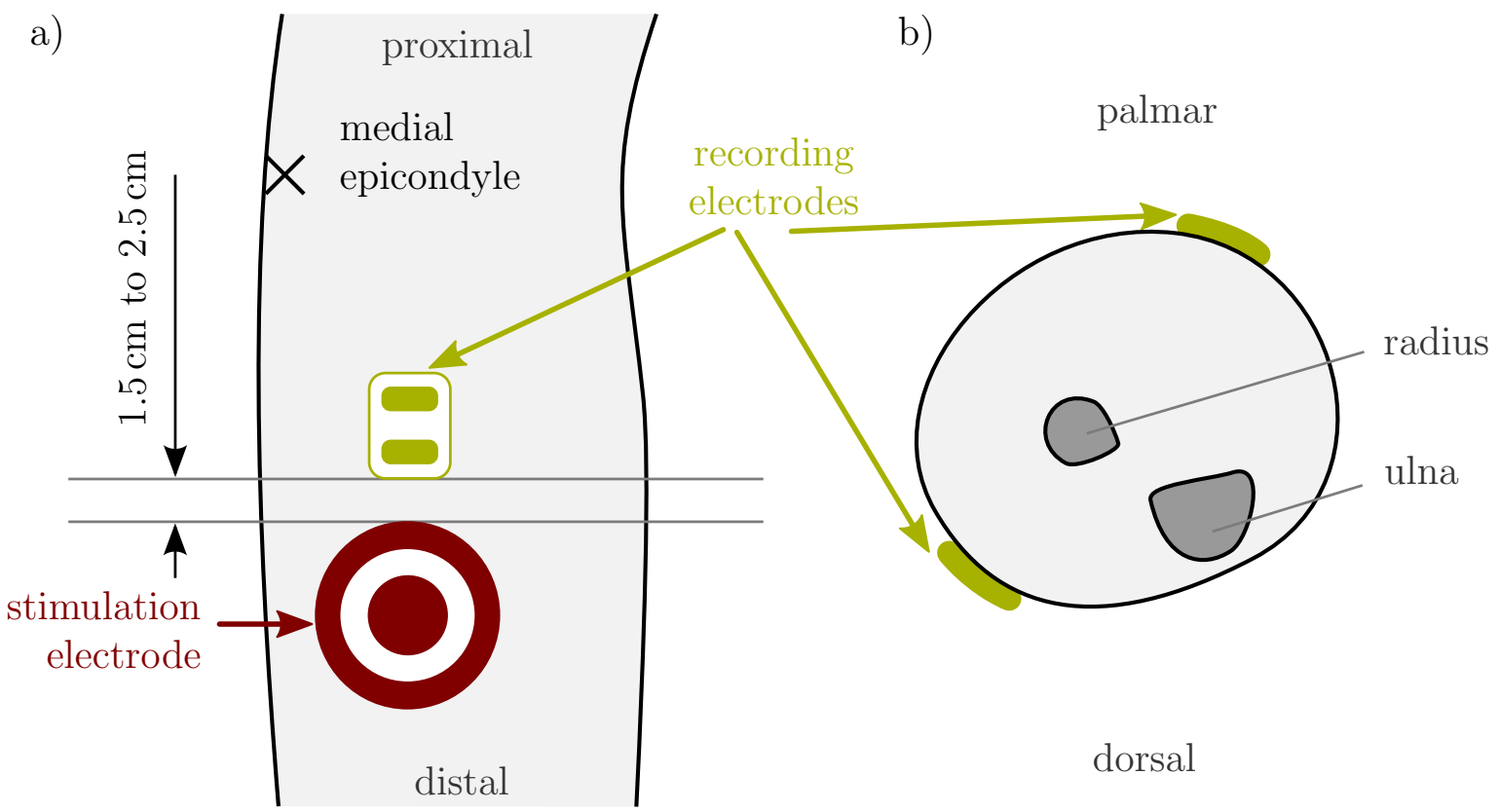

Figure 3.9: Placement of stimulation and EMG recording electrodes: a) Dorsal view of the forearm with recording and stimulation electrodes. b) Transversal view of the forearm illustrating location of recording electrodes. 


\section{INCLUDING MYOCONTROL: THE CHALLENGE OF STIMULATION ARTEFACTS}

The second condition was aimed at determining the influence of the stimulation in the classic proportional control scenario, and thus featured electrocutaneous stimulation. Based on a sensory feedback approach called EMG biofeedback, which was proposed by Dosen et al. [52], the stimulation applied to the subjects' forearm was proportional to the measured EMG activity of the flexor muscles.

The same stimulation was featured in the third condition, which furthermore included additional preprocessing of the EMG signal according to the proposed method of artefact blanking and signal mirroring.

It shall be noted, that this experiment was not focused on determining the usefulness of the feedback itself for this specific task. Its aim was rather to investigate in a practical setup the impact of stimulation artefacts on the prosthesis control and the feasibility of the proposed approach to handle stimulation artefacts.

\subsubsection{Outcome measures}

In order to determine the impact of the stimulation on the voluntary control of the prosthesis, the task performance of the subjects with EMG contaminated by stimulation artefacts and EMG subjected to artefact blanking and signal mirroring were compared to each other and the benchmark performance (no stimulation). Task performance for each run was measured in the number of blocks successfully transferred across the barrier within a $60 \mathrm{~s}$ interval. For statistical comparison, tow-way repeated measures ANOVA and Newman-Keuls post hoc tests were applied. Factors were condition (benchmark, contaminated, treated) and the number the respective condition had been repeated (five runs per condition).

\subsubsection{Results}

The results of the practical application of the artefact handling approach are shown in figure 3.10. In the control scenario without stimulation, subjects were able to move between seven and 25 blocks (average: $16 \pm 4$ ) across the barrier between compartments within the given time frame of 60 seconds. With stimulation but without artefact handling the number of blocks successfully moved ranged from zero to 19 (average: $8 \pm 7$ ). Notably, while for some subjects the control of the prosthesis was not noticeably affected during the stimulation condition as compared to the control condition, for others the prosthesis was no longer controllable due to the prevailing effect of the artefacts. When the proposed approach was applied to eliminate the artefacts from the EMG signal subjects managed to move eight to 25 blocks (average: $16 \pm 4$ ). There was a statistically significant difference between the unhandled condition and the other two conditions $(p<0.001)$, but no difference between the control condition and the test 

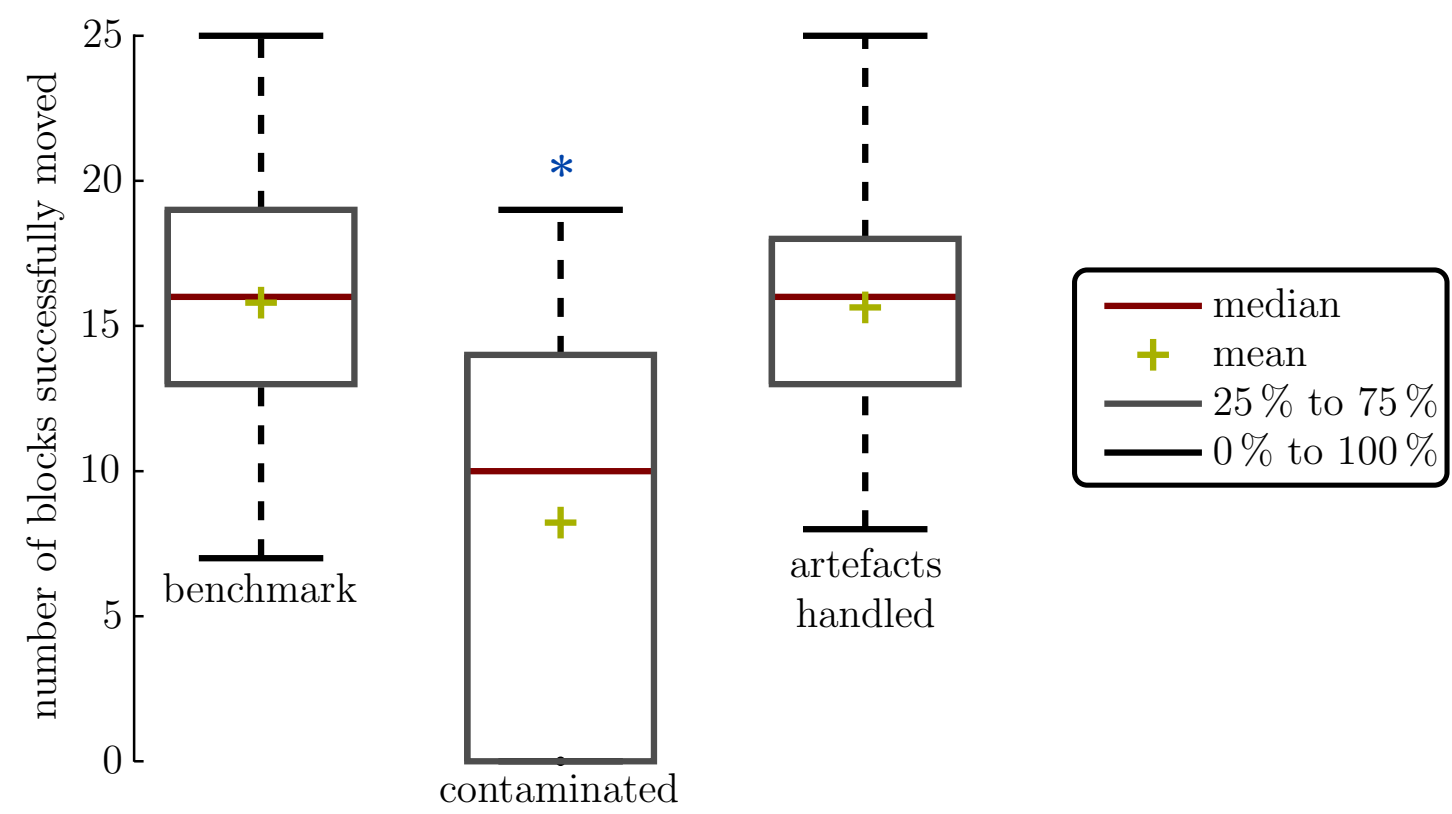

Figure 3.10: Results of the practical evaluation by box and blocks routine. When stimulation was present, artefacts had a detrimental effect on the EMG and subjects could no longer transfer as many blocks from one box to the other. The performance drop was significant $(p<0.001)$. Handling the artefacts restored the performance back to the benchmark. Boxes show 25\% to $75 \%$ percentile, whiskers indicate complete range of measurements.

condition with artefact handling $(p>0.9)$.

\subsection{Discussion}

In this study, the impact of stimulation artefacts on proportional control of prostheses, as well as the classification performance of pattern recognition in multichannel EMG data was investigated. Artefact blanking was proposed as a practical approach to handle stimulation artefacts and to restore performance of both control approaches.

\subsubsection{Pattern Recognition}

A common classifier and four feature sets have been evaluated using experimental data collected in seven subjects. Blanking in combination with three data segmentation methods was proposed and tested for artefact removal because it is 


\section{INCLUDING MYOCONTROL: THE CHALLENGE OF STIMULATION ARTEFACTS}

very convenient for a practical application.

It was shown that the application of any of the investigated approaches significantly improved the classification performance for EMG data contaminated by stimulation artefacts, in some cases restoring the performance to the benchmark level (artefact-free data classification). This is a promising result, indicating that a simple and practical method to deal with the stimulation artefact can be used to close the loop using electrotactile sensory feedback in myoelectric prostheses controlled using pattern recognition.

Both simulations (virtual blanking) and experimental evaluation (actual blanking) revealed that the approaches $\operatorname{VarB}$ and $\operatorname{VarC}$ are better than VarA in terms of classification performance recovery. This was an expected result since the methods VarB and VarC avoided using blanked signal segments (no information) and only used actual, voluntarily generated EMG recorded in between the artefacts. In the experimental evaluation, the average classification rate $r_{\text {class }}$ was consistently higher for the variants VarB and VarC as compared to the approach VarA, although for some feature sets the differences were not statistically significant ('RMS' and 'RMS+FD'). However, for all feature sets, VarB and VarC were more successful in restoring the performance back to the benchmark level (Table 3.6). Similarly, in virtual blanking tests, the methods VarB and VarC outperformed VarA consistently in all the outcome measures (Tables 3.2-3.4), and the differences (VarA vs. VarB or VarC) were statistically significant in all cases, except for the feature set 'RMS+FD' (Table 3.5). In particular, the simulation results indicated that in the more challenging stimulation conditions with higher frequencies and/or longer artefact duration, methods VarB and VarC are likely to result in a better performance (Fig. 3.6). For feature sets 'RMS+TD' and 'RMS+TD+FD', this was confirmed in the experiments as well, where VarB and VarC significantly outperformed the approach VarA for higher active stimulation ratios $(\kappa \geq 6 \%)$.

A somewhat surprising result was that the methods VarB and VarC performed similarly in the virtual blanking (few statistically significant differences, cf. Table 3.5) and almost identically in the applied blanking (no significant differences, cf. Fig. 3.7), although VarB uses considerably shorter data segments for feature extraction (up to $40 \%$ samples discarded). However, this result is in agreement with the findings of Englehart \& Hudgins [46], who demonstrated that using less data indeed decreased the classification performance, but in a rather gradual manner for a certain range of segment lengths. For example, in their experiment (four EMG channels, four classes, LDA, time domain features) the approximate performance difference for the range of segment lengths as in the current study, was no more than $2 \%$. Additionally, the work of Smith et al. [53] as well as Liu et al. [54] indicate that the classification performance is less sensitive to segment length if more EMG channels are used. 
When comparing the performance for the virtual and applied blanking, the trends were similar, but the classification rates were lower in the applied case. The likely reason for this result was that the stimulation slightly affected the EMG signal even outside the segments containing the main contribution of the stimulation artefact. For example, occasional long exponential tails and short term baseline changes in the vicinity of the artefacts could be noticed upon visual inspection. These effects were variable between subjects and trials, localized and of small amplitude. Nevertheless, these minor effects seemed to be sufficient to decrease the classification rate with respect to the simulated conditions.

For all approaches, the ability to achieve a high accuracy depended on the interaction of the following factors: stimulation frequency, artefact duration, and the type of features used. Furthermore, in the case of VarC, the maximum additional delay that may be introduced into the system has to be considered. The results of this study can be used to select the features and a strategy for coping with stimulation artefacts when implementing a closed-loop myoelectric control system using electrotactile feedback. The simplest approach of VarA is not suited for most practical applications since it provides good performance only for the lowest stimulation ratios $(\kappa<6 \%)$. Therefore, the methods $\operatorname{VarB}$ or $\mathrm{VarC}$ should be preferred. The method VarB uses less data for feature extraction while VarC implies a longer delay. The results of this study demonstrated, that using less data did not adversely influence the performance for the range of stimulation ratios used for the applied blanking experiments (i.e., methods VarB and VarC led to similar results). However, the approach VarC added a delay that in the worst case was approximately of $210 \mathrm{~ms}$, which is not acceptable in real-life prosthetic applications. Therefore, the variant VarB is suggested. With this approach, a full performance recovery could be achieved for active stimulation ratios of $3 \%$ for the feature sets 'RMS+FD' and 'RMS+TD+FD', $4 \%$ for the feature set 'RMS+TD', and even $6 \%$ for 'RMS' (Table 3.6). In terms of stimulation parameters, the latter case would, for example, correspond to a maximum tolerable stimulation frequency of $150 \mathrm{~Hz}$ at a pulse width of $400 \mu \mathrm{s}$. For 'RMS+FD' or 'RMS+TD+FD' a pulse width of $200 \mu \mathrm{s}$ could be tolerated at the same frequency. Indeed, $150 \mathrm{~Hz}$ was the maximum frequency used in the experiments. If higher frequencies are used, the stimulation ratio can be used to estimate a pulse width that may still be handled with the blanking. Continuing the above example, using a stimulation frequency of $300 \mathrm{~Hz}$ would limit the maximum recommendable pulse width to $200 \mathrm{\mu s}$ for feature set 'RMS' $(\kappa=6 \%)$, and to $100 \mu \mathrm{s}$ for 'RMS+FD' and 'RMS+TD+FD' $(\kappa=3 \%)$.

In conclusion, while the presence of artefacts in EMG recordings substantially influences the classification accuracy in EMG pattern recognition, this study showed that accuracy can be recovered to the artefact-free levels by simple blanking and rejection of the blanked data. This practical method determined the same perfor- 


\section{INCLUDING MYOCONTROL: THE CHALLENGE OF STIMULATION ARTEFACTS}

mance as in artefact-free conditions for stimulation frequencies as high as $150 \mathrm{~Hz}$ and stimulus duration as long as $400 \mu \mathrm{s}$, which offer large ranges for parameter selection.

\subsubsection{Proportional Control}

Based on these results for pattern recognition for multi-channel prosthesis control, a second task-based experiment was designed to evaluate the impact of electrocutaneous stimulation on classic proportional control of prostheses as it is widely used in current commercial devices. The ability to restore control performance using an artefact rejection and segmenting approach was investigated. The approach was based on the most feasible approach which was identified for pattern recognition, and only used uncontaminated data segments for estimation of the control signal.

Direct proportional control of opening and closing of a commercial hand prosthesis by two EMG channels under three different conditions was performed by 15 subjects in an applied grasp - lift - release task (box and blocks test).

It was shown that electrocutaneous stimulation had a strong detrimental effect on the control performance if artefacts were left unhandled. However, the proposed approach to pre-process contaminated EMG signals proved to be successful to consistently restore the performance back to the benchmark level. As the proposed artefact blanking technique is computationally cheap and has only moderate memory requirements it could easily be implemented with current commercial devices. This facilitates the introduction of prosthetic devices using electrocutaneous stimulation to close the loop to the market, and thus being made available to a large number of amputees in the near future.

That the approach was able to restore the control performance with a proportionally controlled device was an expected result, even slightly exceeding expectations in completely and consistently doing so for every single subject participating in the study. While subjects were able to successfully move an average of $16 \pm 4$ blocks without stimulation (benchmark condition), this number decreased to $7 \pm 6$ when stimulation artefacts were present in the EMG signal. The detrimental effects were more severe for some subjects than for others, which indicates that individual factors such as conductive and capacitive properties of the skin do indeed play a major role in the formation of stimulation artefacts. Independent of the severity of the control limitations, a full restoration to the benchmark performance of $16 \pm 4$ successfully moved blocks when artefact blanking and signal mirroring are used to treat contaminated EMG shows the effectiveness of this practical approach.

In conclusion, while electrocutaneous stimulation detrimentally influences the EMG signal and poses severe limitations in direct proportional control, this ex- 
periment showed that control capability can be fully recovered by simply rejecting stimulation artefacts and using pre-artefact EMG stored in a FIFO buffer.

This study has shown that both classic and advanced myocontrol approaches can successfully be used while electrocutaneous stimulation is applied in the immediate vicinity of the EMG recording sites. Combined with the recommendations on feedback encoding paradigms resulting form the first study, this facilitates closing the loop in EMG-controlled prosthetic systems using electrocutaneous feedback. In fact, within the scope this study a first closed-loop myocontrolled prosthesis system was already implemented and successfully tested during online evaluation of the proposed artefact handling method. The remaining chapters are hence dedicated to the investigation of the role that sensory feedback plays in closedloop prosthesis control. For this, functional as well as non-functional aspects of feedback are considered. The following chapter focuses on the discussion of functional aspects and presents a study on closed-loop routine grasping with a real prosthetic hand under different feedback conditions (amount of feedback). 
3. INCLUDING MYOCONTROL: THE CHALLENGE OF STIMULATION ARTEFACTS 


\section{Chapter 4}

\section{Full closed-loop myocontrol in routine grasping: The role of feedback}

The task of implementing an artificial sensory feedback to close the loop in myoelctric prosthesis control is a challenging one. This is not because of the difficulties of finding optimal solutions for encoding and providing sensory feedback information to a prosthesis user, or the detrimental effects that electrocutaneous stimulation has on the control of prostheses. The previous chapters demonstrated, that these tasks and issues can be successfully solved. The actual challenge lies in the nature of the control loop itself as a whole. This includes the prosthesis user, as well as the prosthesis. The user is in a difficult position: In any case, his intentions are not instantaneously turned into action by the prosthesis. Too many delays in the signal processing chain make this impossible. Analogue/digital converters have sample and hold times, control algorithms rely on data segments that are commonly around $100 \mathrm{~ms}$ to $250 \mathrm{~ms}$ long $[46,47,53]$. That these delays exist, and that they already affect user satisfaction is a well established fact. While a delay of up to $300 \mathrm{~ms}$ in a feedforward-controlled prosthesis is generally assumed to be acceptable $[46,55]$, Farell and Weir determined in an applied test that the maximum controller delay should not exceed $100 \mathrm{~ms}$ to $125 \mathrm{~ms}$, as longer delays already have a detrimental influence on user performance.

When advancing from feedforward control to closed loop control, additional delays are to be expected on the feedback path, analogous to the delays already present in the feedforward path. The result is a comparatively large control delay, and effectively makes the control task extremely challenging for the prosthesis user, who takes the role of the controller in the whole system. Furthermore, it was hypothesised that for fast motions the time required to process any sensory input, e.g. from proprioception, is actually larger than the time window during 


\section{FULL CLOSED-LOOP MYOCONTROL IN ROUTINE GRASPING: THE ROLE OF FEEDBACK}

which the execution of the motions can still be influenced [56]. Functional benefits of artificial sensory feedback may hence be limited to slow tasks.

However, even for mastering fast motions, such as routine grasping or pointing, sensory feedback might still be employed by the prosthesis user reflectively, i.e., in order to continuously improve his prosthesis control on a trial-by-trial basis.

To test this hypothesis, a study was designed in order to investigate how learning to use a prosthesis to grasp objects with different slip and fragility properties is influenced by the amount of feedback available to the user. Three different feedback conditions were compared: In a basic feedback scenario, only feedback on the outcome of the grasp attempt was provided, as well as indirect visual and auditory feedback available from the prosthesis hand. In two further conditions, additional feedback on the currently exerted grip force was supplied. In a visual condition, this was supplied via a visual representation on a screen, which is easy to interpret and was regarded as ideal feedback representation. In a tactile condition, the same information was provided via an intensity- and frequencymodulated tactile stimulus.

Few studies specifically addressed the role of feedback in fast routine grasping tasks. In a recent study by Dosen et al., explicit grasp force feedback was provided to subjects via a visual channel [57]. One aspect of the study addressed the realistic scenario of controlling a real prosthetic hand by EMG signals. Subjects were successful in utilising the presented feedback when asked to repeatedly perform fast grasps with specific target force. However, after depriving subjects of the feedback again, the performance was found to deteriorate, and drifting of the applied force was observed. It shall be noted, that in this study, neither visual nor auditory perception of the prosthesis was available to the subjects, so that when deprived of the artificial feedback, they had to exclusively rely on the previously build feedforward model for control and their proprioceptive feedback. However, this is not a realistic scenario, as in real life prosthesis users usually receive a basic visual (direct observation of hand and interaction objects) and auditory feedback (perception of motor noise). For instance, the deformation of grasped objects and of prosthetic fingers indicates the magnitude of interaction forces or grip strength. This feedback may to a certain degree be exploited. Furthermore, the possibility to observe the outcome at each and every grasp attempt represents a form of basic feedback as well.

Ninu et al. investigated this intrinsic feedback and its role in routine grasping. They also identified an additional feedback variable available to the user: the observed speed with which the hand closes on the object [58]. The prosthetic system they used in their study had the property of generating a grasp force that is proportional to the closing speed. Therefore, they hypothesised that the observed speed is a predictor for the force, and can thus be employed by the subject. This assumption could be confirmed by the results of the study. However, they 
conceded that proportionality between closing speed and grasp force is essential for this. Depending on the prosthesis and the control approach employed, this may not be the case in real life.

The study presented here attempted to investigate the role of additional sensory feedback for learning of a feedforward task in a more realistic scenario. Subjects had the opportunity to exploit any visual and auditory feedback cues from the prosthesis just as an amputee could in daily use of his device. Additionally, target force ranges were neither explicitly nor implicitly presented to the participants. Rather a representation of two ordinary everyday objects was presented, and the amount of force to successfully handle these objects had to be figured out in a series of trial and error. Participants received an equivalent of the naturally available feedback on the success of the grasp attempt, i.e., they were informed if the object would have slipped from their grasp due to insufficient grasp force, or even been destroyed due to excessive application of force. While this basic feedback was available across all conditions, explicit feedback on the currently applied grip force was only available to the subjects in the two extended feedback conditions (visual and tactile).

\subsection{Methods}

In this study, a commercially available prosthetic hand prosthesis was controlled by the participants to repeatedly grasp a dummy object. For repeatability of grasp conditions and object contact, the prosthesis was placed on a stand on the table instead of being worn by the subjects. Hence, the usual spatial constraints regarding placement of the stimulation end effector within a closely fitting prosthetic socket did not apply, and for ease of setup and shortening of the experimental time for each subject, vibrotactile stimulation was chosen over electrocutaneous stimulation. In electrocutaneous stimulation, subject-specific sensation and comfort thresholds need to be determined, which constitutes a certain mental effort for the subjects and would additionally have prolonged the protocol. However, since for this experiment it was essential to keep subject concentration on a high level throughout the protocol, the possibility to shorten the setup time by using vibrotactile stimulation was exploited. An effect on the outcome of the experiment due to the modality of the tactile stimulation is not expected, as it was previously shown that vibrotactile stimulation and elctrocutaneous stimulation perform similarly as feedback modality [59]. 


\section{FULL CLOSED-LOOP MYOCONTROL IN ROUTINE GRASPING: THE ROLE OF FEEDBACK}

\subsubsection{Experimental Setup and Protocol}

For this study a commercial state-of-the-art prosthetic hand (Michelangelo hand prosthesis, Otto Bock HealthCare GmbH, Germany) was used to grasp a rigid dummy object. The prosthesis features an internal sensor to measure the current grasp force. The measured force can be requested via a specific research protocol and is available in percent of the maximum force applicable by the hand. The closing speed of the prosthesis was controlled by the subjects by an electromyographic (EMG) signal recorded via a single bipolar EMG electrode $(13 \mathrm{E} 200=50 \mathrm{AC}$, Otto Bock HealthCare GmbH, Germany) from the wrist flexor muscle of the left forearm. For this, the root mean square value over $128 \mathrm{~ms}$ of EMG signal was calculated, and was used to proportionally control the closing speed of the prosthesis. Subjects were informed prior to the experiment that by steadily contracting their forearm muscles with a certain intensity, the hand would close on the dummy object with a constant speed, and the final force that would be measurable at the dummy object when the grasp was completed would be approximately proportional to the contraction intensity they had chosen. The task was to quickly grasp the dummy object with a certain target force. Once the object was grasped, the forward control was disabled to prevent user attempts at correcting the applied force. There were two target force ranges. The lower target force to be achieved simulated to grasp a light, fragile object, such as a raw egg. The higher target force represented the force necessary to handle a heavy, robust object, e.g. a dumbbell. However, subjects were left ignorant of the valid force ranges that would correspond to successful object manipulation. The only cue they got was a visual representation of the simulated object they should attempt to grasp. Subjects had to find the correct force, and with that the correct amount of muscle contraction on a trial-and-error basis.

Three types of feedback were designed for the purpose of this experiment. Auditory feedback on the overall success of a single grasp attempt, and both visual and tactile feedback on the current grasp force exerted by the prosthesis. Visual feedback was intended as mean to establish a benchmark performance, as it is could be considered to be an ideal feedback where the feedback intensity is easily and intuitively understood due to good visual resolution and a certain accustomedness to visual signal representations in daily life (e.g. analogue thermometers, speedometers, clocks, scales ...). Tactile feedback was intended to carry the additional information content on the grasp force not usually available to prosthesis users.

In order to determine the training success over time, the experiment was split into seven runs with five minute breaks between runs. Each run consisted of 50 grasp attempts. As mentioned earlier, the goal of each grasp was to close the prosthesis on the dummy object, so that the force applied to the object was 


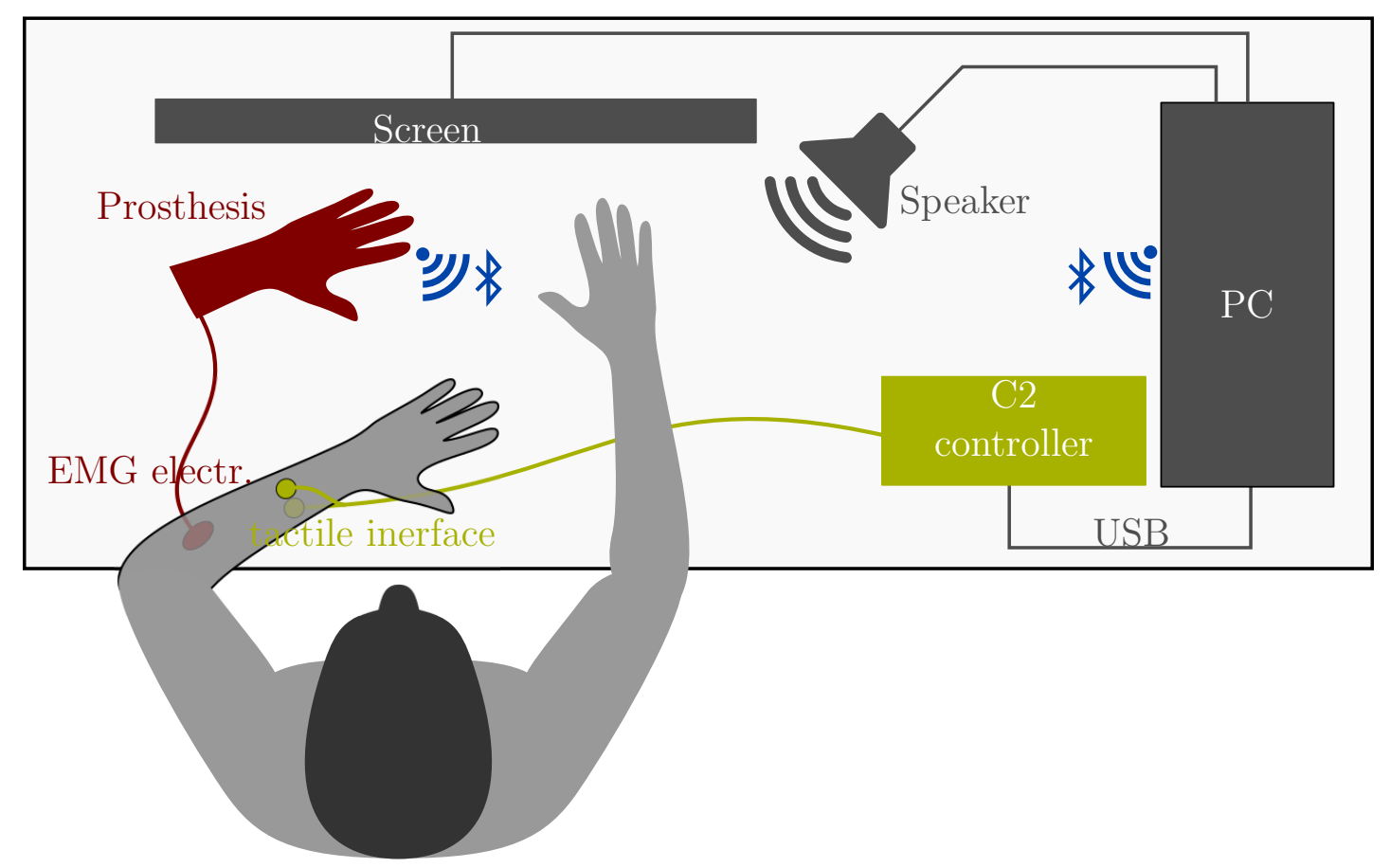

Figure 4.1: Schematic of experimental setup as seen from above. A bipolar EMG electrode records the flexor muscle signal used to control the prosthetic hand. Next to the EMG electrode and on the opposite side of the forearm two vibration tactors are applied. Sensor data from the prosthesis is transmitted to the PC via Bluetooth. The task is presented to the subject on the screen. Basic feedback on the success of each grasp attempt is provided via the speaker.

within a certain range. The two force windows targeted in this experiment were $35.0 \% \pm 7.5 \%$ of the prosthesis's force range (representing a light, fragile object) and $67.5 \% \pm 7.5 \%$ (representing a robust and heavy object). Of the 50 grasp attempts in each run, 25 trials were aimed at the lower target force, and 25 trials at the higher target force. Low and high force targets were presented in random order under the constraint that at least two consecutive trials had the same target force window.

A schematic view of the experimental setup is represented in figure 4.1, the view as seen by the subject during the experiment is shown in figure 4.2.

\subsubsection{Participants and conditions}

In order to investigate the effect of different depths of information content about grasp force on the training of a routine grasping task, three different feedback 


\section{FULL CLOSED-LOOP MYOCONTROL IN ROUTINE GRASPING: THE ROLE OF FEEDBACK}

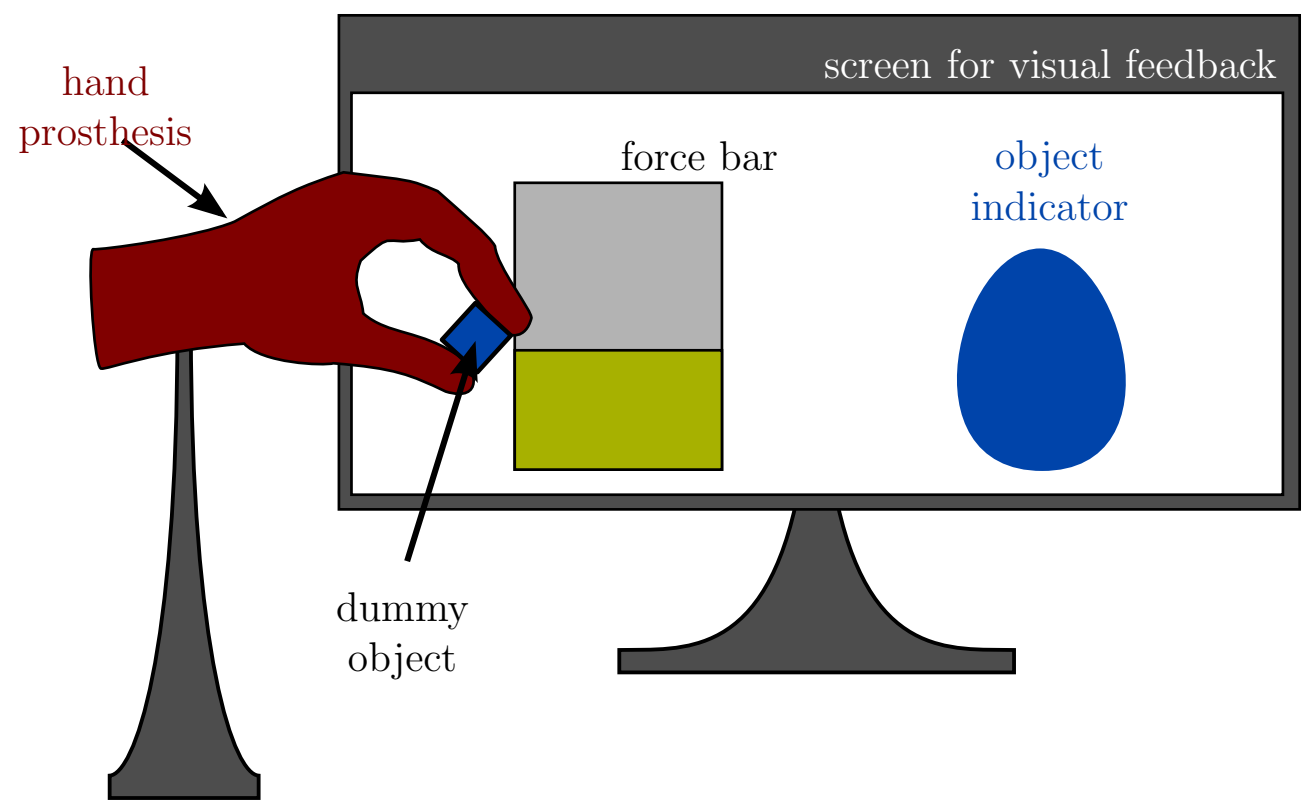

Figure 4.2: Schematic of the view presented to the subject during the experiment. On a computer screen the target force window is indicated by a visual cue (object indicator). For the visual feedback group, a visual representation of the current grasp force (force bar) is presented on the screen next to the object indicator. The prosthesis hand is placed in front of the screen. 
conditions were designed for this study. Subjects participating in the experiment were distributed into three groups, where each group performed the experiment under one of the feedback conditions, i.e., different subjects received different types of feedback about the grasp force that was exerted with the prosthesis.

A total number of 22 able-bodied subjects (13 male, 9 female, average age $24 \pm 4$ years) volunteered to participate in this study. During the experiment, it became obvious, that one of the subjects had not understood the task, and that data collected from this subject would potentially falsify the outcome of this study. Hence, this data was excluded from the analysis later on, and a replacement subject was recruited to maintain even group sizes.

The first group, the VISUAL feedback group, received a visual feedback via the computer screen. The feedback was displayed as a bar graphic, where the height of the bar was proportional to the grasp force measured at the prosthesis. The second group, the TACTILE group, received a vibrotactile feedback, which was transmitted to them via two C2 tactors (Engineering Acoustics Inc., USA), which were placed on the ventral and dorsal side of the forearm close to the EMG recording electrode (compare figure 4.1). Two stimulation sites were used instead of one, as findings of the first study indicated that the use of more than one stimulation channel improves the perceptibility of the feedback (cf. chapter 2). The vibrations were modulated in order to represent the prosthesis's current grasp force. This study also focuses on temporal aspects of mastering a new skill, and it was assumed that with time, subjects could exploit tactile grasp force feedback encoded via intensity. This directly relates to findings of the first study and represents a further application the results. Additionally, frequency of the vibration was mapped proportionally to the full range of force achievable with the prosthesis. Here, the minimum detectable force corresponded to a $50 \mathrm{~Hz}$ vibration with $20 \%$ of the possible amplitude, and the maximum force was mapped to a $200 \mathrm{~Hz}$ vibration with $100 \%$ of tactor amplitude. The third group, the BASIC group, did not receive any information about the exact force that was exerted by the prosthesis. After each grasp attempt, members of each group received an auditory cue, which informed them of the overall success of the current grasp attempt. Thus they received the basic information if the achieved force was appropriate for the object, too high (object would have been broken), or too low (object would have slipped during manipulation). This audio cue conveys the outcome of the grasp attempt that can in any case be perceived in real life, with one's own hands as well as with conventional prosthetic hands.

\subsubsection{Outcome measures}

As a measure for the subjects' performance, success rate was used. It is defined as the ratio of successful grasp attempts over all attempts in a run. For each run, 


\section{FULL CLOSED-LOOP MYOCONTROL IN ROUTINE GRASPING: THE ROLE OF FEEDBACK}

Table 4.1: Low force grasp success rates

\begin{tabular}{cccc}
\hline run & BASIC & VISUAL & TACTILE \\
\hline 1 & $62.9 \% \pm 10.8 \%$ & $63.4 \% \pm 16.6 \%$ & $60.0 \% \pm 14.4 \%$ \\
2 & $62.9 \% \pm 14.2 \%$ & $70.3 \% \pm 11.7 \%$ & $57.7 \% \pm 14.9 \%$ \\
3 & $68.0 \% \pm 8.0 \%$ & $72.6 \% \pm 18.7 \%$ & $61.7 \% \pm 13.8 \%$ \\
4 & $68.6 \% \pm 15.2 \%$ & $74.9 \% \pm 20.9 \%$ & $69.7 \% \pm 14.2 \%$ \\
5 & $69.7 \% \pm 8.6 \%$ & $73.1 \% \pm 12.8 \%$ & $75.4 \% \pm 13.2 \%$ \\
6 & $74.3 \% \pm 12.6 \%$ & $73.7 \% \pm 16.6 \%$ & $67.4 \% \pm 17.0 \%$ \\
7 & $67.4 \% \pm 14.7 \%$ & $73.7 \% \pm 12.8 \%$ & $64.6 \% \pm 11.6 \%$ \\
\hline
\end{tabular}

Table 4.2: High force grasp success rates

\begin{tabular}{cccc}
\hline run & BASIC & VISUAL & TACTILE \\
\hline 1 & $26.3 \% \pm 8.3 \%$ & $26.9 \% \pm 8.9 \%$ & $30.3 \% \pm 13.6 \%$ \\
2 & $33.7 \% \pm 21.3 \%$ & $33.7 \% \pm 14.2 \%$ & $38.3 \% \pm 12.8 \%$ \\
3 & $38.3 \% \pm 18.6 \%$ & $37.7 \% \pm 12.0 \%$ & $45.7 \% \pm 20.5 \%$ \\
4 & $40.6 \% \pm 12.1 \%$ & $45.1 \% \pm 18.6 \%$ & $46.3 \% \pm 10.3 \%$ \\
5 & $39.4 \% \pm 17.5 \%$ & $34.3 \% \pm 8.3 \%$ & $37.7 \% \pm 19.4 \%$ \\
6 & $41.7 \% \pm 16.5 \%$ & $42.3 \% \pm 13.6 \%$ & $50.9 \% \pm 12.2 \%$ \\
7 & $43.4 \% \pm 23.5 \%$ & $44.6 \% \pm 17.2 \%$ & $45.7 \% \pm 14.0 \%$
\end{tabular}

success rates for low and high target force windows were determined separately. Grasp force variability as measured by the standard deviation of final grasp forces per grasp attempt, was employed as a measure of the subjects' ability to reliably reproduce grasps. Repeated measures three-way ANOVA with the force level and number of run as within-subject factors, and feedback condition as categorical factors, as well as Newman-Keuls post hoc tests were applied to statistically analyse success rate and force variability results.

\subsection{Results}

Figure 4.3 shows the success rates for the high force level grasp attempts and the low force level grasp attempts as achieved by the subjects in the different feedback groups. Detailed results are given in tables 4.1 and 4.2, respectively. There were no interactions between the factors (run, force level, feedback con- 

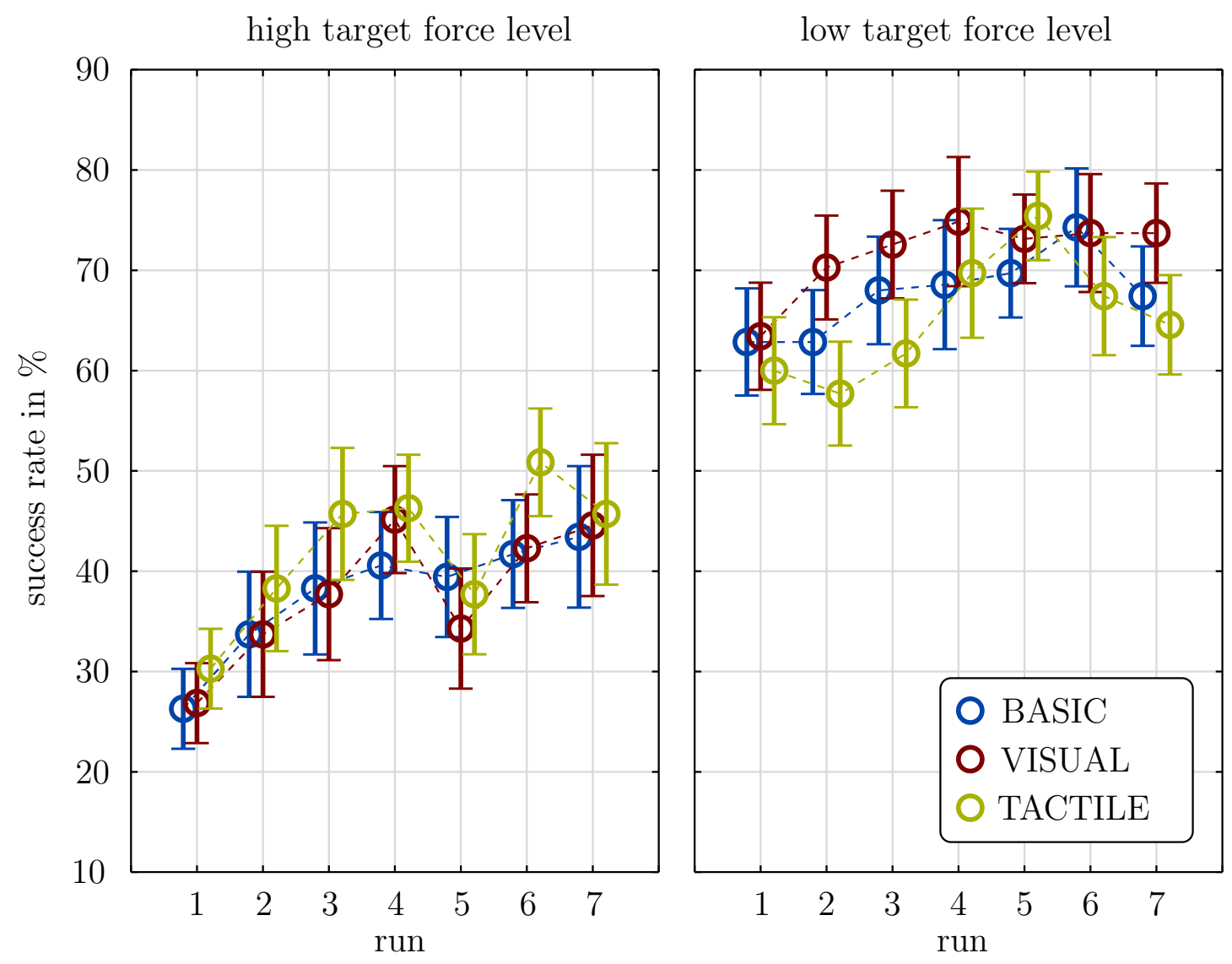

Figure 4.3: Average success rate per run and feedback condition. Success rates for hitting low target force windows are significantly higher than those for hitting high target force windows $(p<0.001)$. No statistical differences between groups were found $(p>0.8)$. 


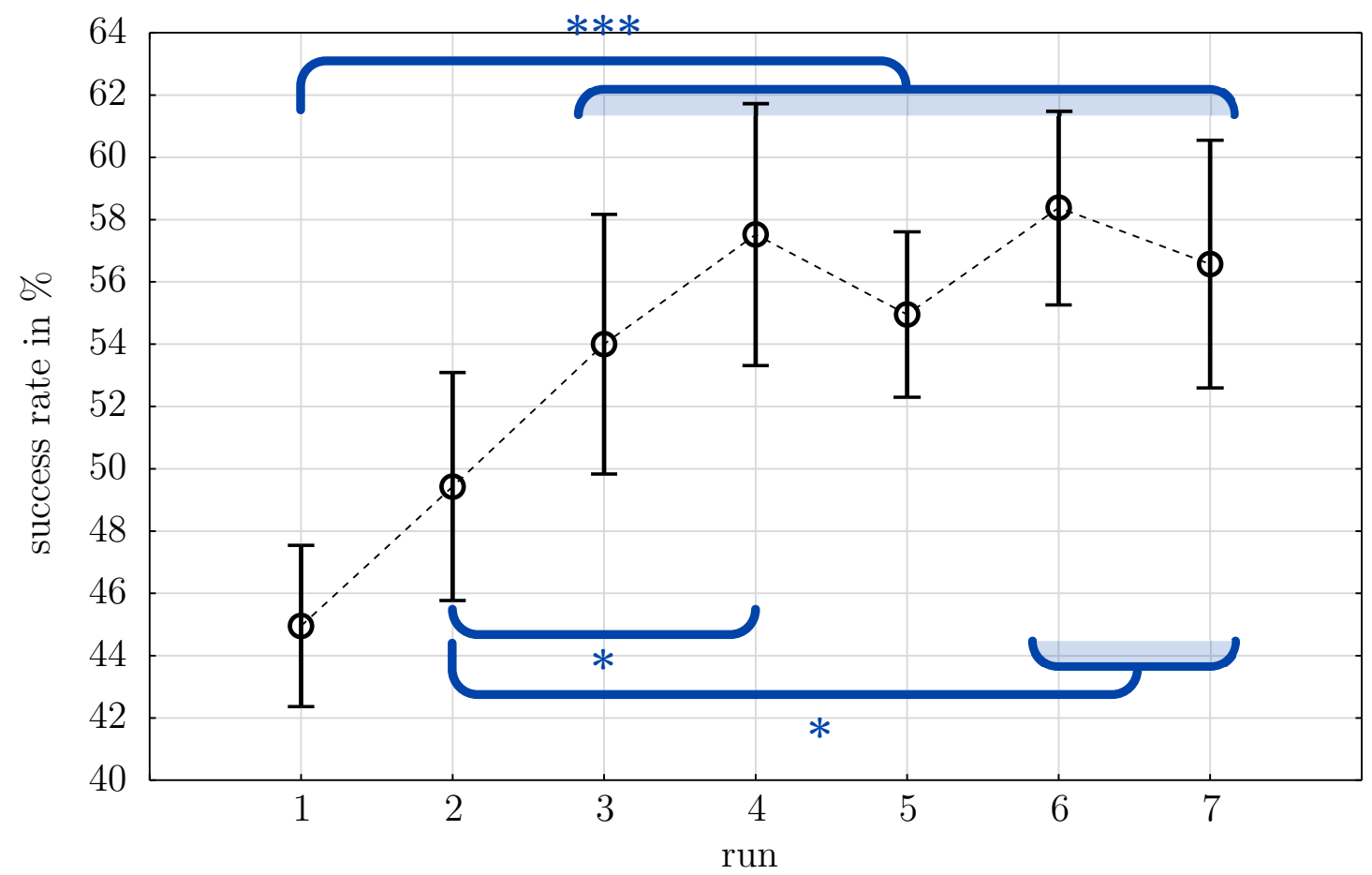

Figure 4.4: Average success rate in each run across all feedback conditions and force levels. Subjects were able to improve their performance with time. 
dition). Also, no difference could be determined between the different feedback conditions.

There was, however, a significant difference in subject performance regarding the force level. In general, subjects were markedly more successful in producing low force grasps than high force grasps $(p<0.001)$. For instance, the minimum success rate measured for the high force level was as low as $4 \%$, while for the high force level at least $36 \%$ of the grasp attempts in each run were successful. The maximum success rate for the high force level achieved during the experiment was $88 \%$, while for the low force level even $100 \%$ were achieved on one occasion. The average success rate across all subjects and runs was $39.2 \% \pm 15.7 \%$ for high force grasp attempts, and $68.2 \% \pm 14.2 \%$ for low force grasp attempts.

Furthermore, subjects were able to improve their performance with time. Figure 4.4 shows the average success rates across all subjects and feedback conditions as determined for each run. While the average overall success rate during the first run was $45.0 \%$, it steadily increased during the following runs until reaching a plateau at roughly $57 \%$ in run 4 . There is a trend for this improvement of grasp success to be more pronounced for the high target force level, especially during the first four runs of the experiment, as can be seen from Fig. 4.3.

The variability of the grasp force is shown in Fig. 4.5. As for the success rate, there were no interactions between the factors feedback condition, number of run, and target force level. Again, no statistically significant difference between the different feedback conditions could be determined. However, similar to the observations made for the subjects task performance, both the main factors number of run and force level were found to influence the variability of the grasp force. Grasp attempts targeting the higher force level were significantly more variable than those targeting the lower force level $(p<0.001)$. While variability for high force grasps ranged from $10.7 \%$ to $16.7 \%$ of the prosthesis's total grasp force range, variability for low force grasps was only in the range of $6.3 \%$ to $10.5 \%$. In average, variability of grasp forces decreased significantly after the first run, which can be clearly seen in Fig. 4.6. Initially, average force variability was larger than $13 \%$ of the whole force range the prosthesis could produce. By the second run, subjects were able to decrease variability by $20 \%$, and by $27 \%$ by the final run.

Finally, for high target forces, there was a trend for lower force variability to emerge after the first two runs in the TACTILE group as compared to the BASIC and VISUAL feedback groups. While decrease of force variability was comparable for all groups between the first and second run, in the third run the average force variability only continued to decrease further with the tactile feedback. Henceforward, it was consistently lower in this group than for the other feedback conditions. This can be seen in Fig. 4.5. . However, the difference was not statistically significant $(p>0.05)$. 


\section{FULL CLOSED-LOOP MYOCONTROL IN ROUTINE GRASPING: THE ROLE OF FEEDBACK}
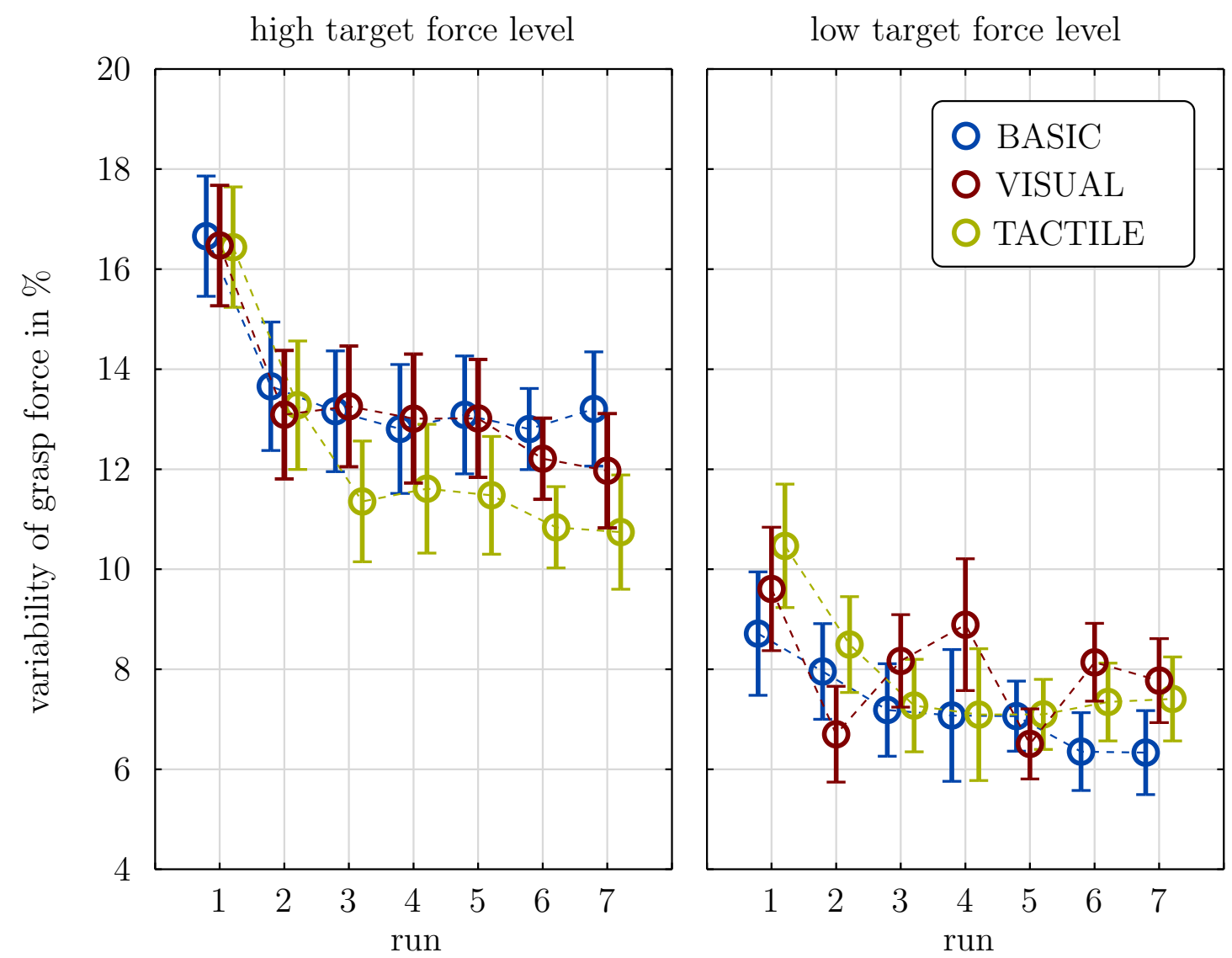

Figure 4.5: Variability of grasp force within each run. There is less variability in low force grasps than in high force grasps $(p<0.001)$. Variability significantly decreases after the first run for all feedback conditions in the high target force grasps. There was a trend that for high target forces, tactile feedback reduces variability further than basic or visual feedback (run 3 to 7 ). This was, however, not statistically significant $(p>0.05)$. 


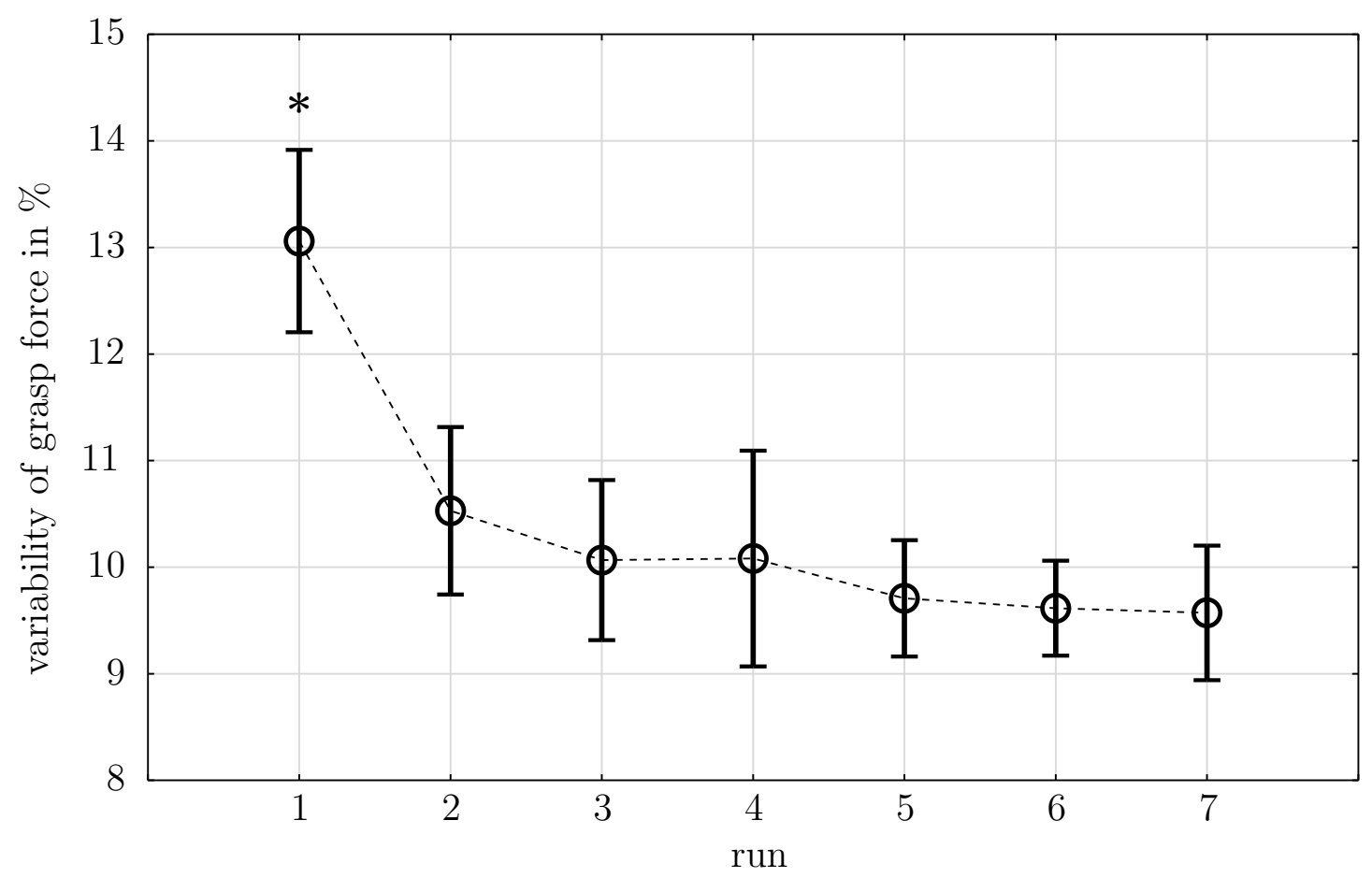

Figure 4.6: Average variability of grasp force across all feedback conditions and target force levels. Variability decreases significantly after the first run $(p<$ 0.001). 


\section{FULL CLOSED-LOOP MYOCONTROL IN ROUTINE GRASPING: THE ROLE OF FEEDBACK}

\subsection{Discussion}

This study investigated the influence of the amount of feedback available on the ability of subjects to master a routine grasping task in the context of objects of previously unknown weight and friction properties. For this purpose, subjects used EMG to control a real prosthetic hand to repeatedly grasp a dummy object. Visual prompts indicated that the object was either heavy and robust (dumbbell), or light and fragile (egg). For a grasp to be successful, the grasp force had to lie within a specific target force range unknown to the subject. Subjects learned to adjust the control of the prosthesis on the basis of trial and error. At that, three different feedback conditions applied. The first group of subjects only had BASIC feedback at their disposal, i.e., they could watch the prosthesis, and post-grasp auditory cues indicated the success of the grasp (force too high, force too low, force appropriate). Two independent groups of subjects received an additional feedback on the currently exerted grasp force via a VISUAL and TACTILE channel, respectively. Success rate and variability of force were used to evaluate subject performance.

Strong differences were found in both success rate and force variability for the different target force levels. This indicates, that the task of reliably producing high grasp forces within a certain range was much more challenging for the subjects than producing lower grasp forces. A learning effect could be observed regardless of the amount of feedback available to the users. That is, improvement of both consistency and success to produce required force could be improved with time. The observed learning effects seemed more pronounced in high force level targets than in low force level targets. Additionally, in the high force condition there was a trend for members of the TACTILE feedback group to continue reduction of force variability and produce more consistent grasp forces, when a plateau had been reached by members of the other feedback groups.

The experiment demonstrated, that the effects of additional artificial sensory feedback were low and hardly contributed to improving or accelerating the subjects' mastering of this specific task. However, in this study, it was not possible for subjects to modulate the grasp force once they had grasped the object, as fast routine grasping should be emulated. In real life, such modulations would be possible, and are indeed performed when an object is not grasped firmly enough and applied force is not sufficient to keep it from slipping during manipulation. The required force depends on weight, friction properties, and manipulation speed, e.g., waving or shaking an object requires a firmer grip than carefully lifting it off a surface. It is assumed, that in such scenarios, the functional advantage of force feedback is more pronounced, and this should be investigated further.

An interesting finding was that high target forces were generally harder to achieve than low target forces, and it can be assumed that this is related to feed forward 
control of the prosthesis. The cause might be the missing capability of the users to reproduce a reliable EMG control signal of appropriate strength. One solution to address this issue has recently been presented by Dosen et al., who suggested using the EMG signal itself as a novel feedback approach to improve prostheses forward control [52]. Thus, users could immediately assess the strength of the control signal they are producing, without having to derive it from the behaviour of the prosthesis.

In conclusion it was shown, that basic feedback suffices to facilitate learning of fast routine grasping in a myocontrolled prosthesis. A trend for tactile feedback to assist in the reduction of force variability was visible, but not significant. Importantly, providing additional sensory feedback beyond the basic feedback already available in real life, did not have detrimental effect on the mastering of the task. This is a fundamental requirement when considering the application of sensory feedback in non-functional contexts. Such a non-functional context could be the use of electrocutaneous stimulation to aid embodiment of prosthetic devices, and related to that, the treatment of phantom limb pain [60]. The following chapter presents a study investigating the role of sensory feedback via electrocutaneous stimulation for facilitation of embodiment of an artificial finger. 
4. FULL CLOSED-LOOP MYOCONTROL IN ROUTINE GRASPING: THE ROLE OF FEEDBACK 


\section{Chapter 5}

\section{Electrocutaneous feedback to facilitate embodiment of prosthetic devices}

In the previous chapters it was demonstrated how to encode information into an electrocutaneous stimulation pattern, so that it is perceived well and can be exploited by the user in a challenging control task. Simultaneous electrocutaneous stimulation and myocontrol of prosthesis has been addressed. It was shown that the two are compatible if appropriate approaches are implemented to handle the challenge of stimulation artefacts. Consequently, functional aspects of sensory feedback were highlighted in the context of closed-loop routine grasping.

The current chapter now addresses a non-functional aspect of sensory feedback in prosthetics, and specifically sensory feedback via electrocutaneous stimulation. A much discussed topic in the context of prosthesis use is the amputee's capability to integrate the prosthetic device into his body scheme. This is called embodiment.

A popular experiment addressing embodiment is the rubber hand illusion, which was first described by Botvinick and Cohen [61]. For this experiment, a lifelike rubber hand is placed in front of a subject and partially covered by a piece of cloth, which is also draped over the subjects shoulder. The setup is arranged in a way that it might be anatomically possible, that the hand protruding from the cloth is actually the hand of the subject. The subject's real hand is instead placed either under the table, or next to the rubber hand, but is in any case shielded from the view of the subject. An investigator then simultaneously stimulates both the rubber hand and the subjects real hand, e.g., by gentle brush strokes. It is important that the stimulation on both real and rubber hand is done in the same location, e.g., a specific digit, and that it is executed in a simultaneous fashion. The subject can feel the stimulus on his own hand, but can only see the 


\section{ELECTROCUTANEOUS FEEDBACK TO FACILITATE EMBODIMENT OF PROSTHETIC DEVICES}

rubber hand. After a time, subjects generally start to perceive the rubber hand as their own hand, and the rubber dummy is temporarily incorporated into the subject's body scheme. An important factor for the success of the illusion is the multisensory stimulation, i.e., visual and tactile. The illusion cannot be created if the stimuli are asynchronous or the rubber limb is positioned in an anatomically implausible position $[62,63]$.

Interestingly, it is not necessary for the stimuli to express the same modality to achieve the illusion of ownership of the rubber hand. In an experiment where subjects' finger tips were stimulated with vibrators instead of a brush, participants still experienced ownership of the rubber hand, which was stimulated by brush strokes as in the classic setup [64]. In another study, Mulvey et al. used a strong electrocutaneous stimulation above the superficial radial nerve to project a feeling of paresthesia into the fingers [65]. It was found that even for this approach to provide a stimulus to the subject's own hand, perceptual embodiment could be achieved, albeit not as consistently as when brush stroking was used.

In the past, variations of this rubber hand illusion experiment were also used to investigate if amputees could perceive this illusion as well, either for rubber hands, or for prosthetic hands which were rather robotic-like in their appearance [62]. Ehrsson and colleagues had amputees participate in a rubber hand illusion experiment, where a rubber hand and the residual limb, and here in particular corresponding points on the phantom map if one was present, were brushed simultaneously [66]. Their results suggested, that amputees could in general experience the rubber hand illusion, and that the presence of a phantom map might assist in this.

The goal of the study presented here was to investigate if an artificial body part, in this case a single digit, could be incorporated into the body scheme if not only the modality of the sensation changed (sensory substitution), but if also the location of the perceived stimulus was incongruous with the visual perception of the stimulus. This is motivated by the fact, that not all amputees express a phantom map on their stump to be used for transfer of sensory information from the prosthesis to the user. Even for those amputees who display a phantom map, it might still be unusable if it superimposes the optimal EMG recording sites. Both cases would require sensory stimulation in sites on the residual limb which are initially not associated with the location on the prosthesis or former hand where a stimulus occurs. However, the working hypothesis here is that amputees learn to interpret the stimuli [3], and that with time plastic changes along the neural pathway will allow them to intuitively associate the skin areas receiving the sensory stimulation with corresponding sites on the prosthesis.

The rubber hand illusion experiment has been modified to include both the aspects of changing the modality of the stimulation, as well as the visuo-tactile incongruence in perceived location of the stimulus. Instead of a rubber hand, a 


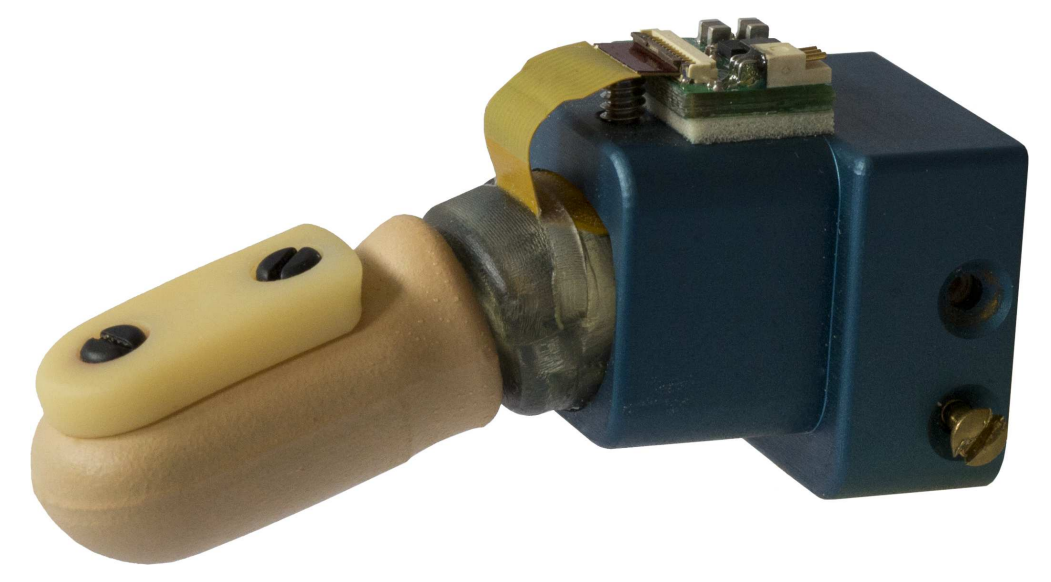

Figure 5.1: The BioTac artificial finger in a special socket used for mounting. The miniature circuit board seen on the socket is for interfacing the finger. The rubber skin has a lifelike paint cover specifically applied for this experiment.

single artificial sensor finger segment was used, and represented the distal phalanx of the index finger. The finger could be interfaced with a computer for collection and processing of sensor data about the investigator's interaction with its skin. As a location for the transfer of stimuli to the subject, the palmar side of the forearm was chosen. In an amputee, this would correspond to the stimulation of the palmar side of the residual limb. Stimuli were presented via electrocutaneous stimulation, as this would be a convenient way of presenting sensory feedback to amputees, as has been discussed earlier. Participants in the study were assigned to one of two groups. While the test group received stimuli that were causally coherent with the stimulation observed for the artificial finger, members of a control group received incoherent and delayed stimuli. By subjecting both groups to an equal amount of electrocutaneous stimulation, any effects of the stimulation itself which are unrelated to the illusion experiment could be excluded.

\subsection{Methods}

\subsubsection{Experimental setup}

In this study, the BioTac artificial finger (Syntouch, LLC, USA, see figure 5.1) was used instead of a complete rubber hand or hand prosthesis. This single artificial digit consists of a rigid core structure, which is equipped with 19 distance sensors, a plastic finger nail, which is connected to the core structure, and a loosely fitting rubber skin. The space between core and skin is filled with a fluid and slightly 


\section{ELECTROCUTANEOUS FEEDBACK TO FACILITATE EMBODIMENT OF PROSTHETIC DEVICES}

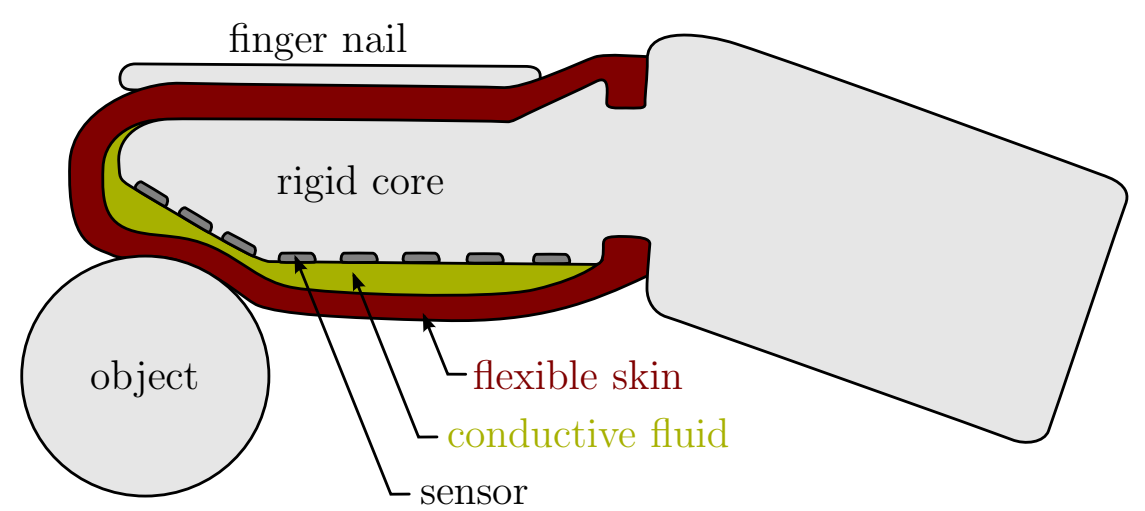

Figure 5.2: Schematic illustration of the artificial finger's structure. Interaction with the finger deforms the skin. This deformation can be detected by the distance sensors attached to the core structure. Due to the total number of 19 sensors a good spatial resolution of the interaction can be obtained.

pressurized. The sensors on the core structure measure the deformation of the rubber skin that occurs when the finger is touched or used to interact with other objects. The internal structure of the finger is shown in the schematic figure 5.2. A green-coloured skin is originally provided with the finger. As in preliminary tests, subjects stated that this unnatural color constantly reminded them that the finger was not a part of their body, a lifelike skin-coloured paint cover was applied to the rubber skin for this study (cf. figure 5.1).

The artificial finger was attached near the edge of a narrow table, the finger tip was pointing diagonally upwards and towards the investigator, who was seated in front of the table. Participants were seated at the opposite side of the table and facing the investigator. Their right forearm was comfortably resting on the table top, palm facing upwards. Participants were directed to place their hand in the proximity of the artificial finger and were asked to extend their index finger in a relaxed fashion, which led to similar postures of both artificial and real finger. A cover was placed over the subject's forearm, so that their own hand was shielded from their view. The artificial finger, however, was protruding from the cover and could be observed by the subjects throughout the experiment. A schematic drawing of this setup is shown in figure 5.3.

Four concentric self-adhesive $\mathrm{Ag} / \mathrm{AgCl}$ electrodes were attached to the ventral side of the forearm. Electrocutaneous stimulation patterns were generated by a medical stimulation device (RehaStim, Hasomed, Germany). Both the sensor finger and the stimulator were connected to a PC running Matlab Simulink and an appropriately configured model implementing the test environment described 


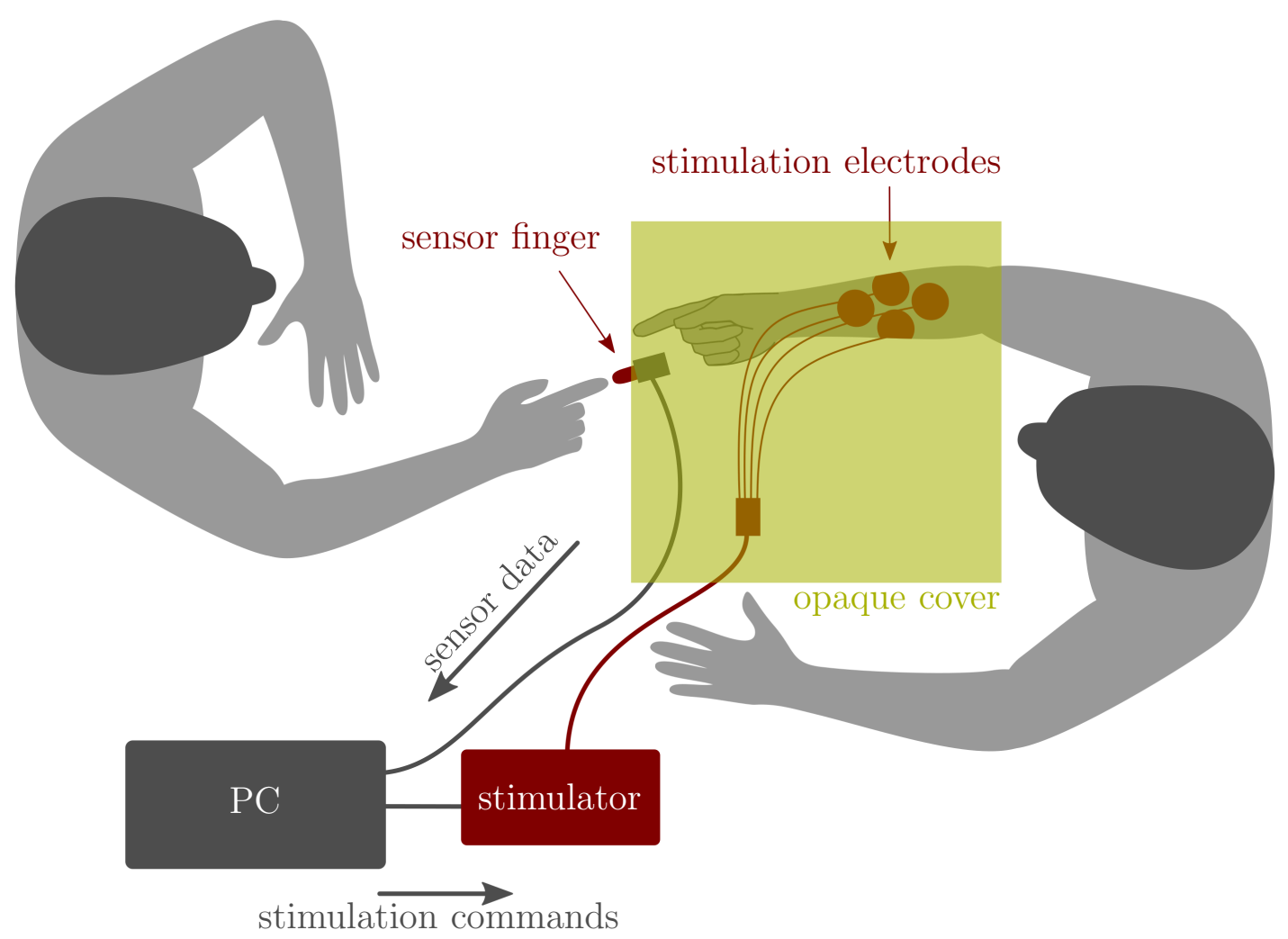

Figure 5.3: Schematic drawing of the experimental setup. The investigator interacts with the sensor finger, while the subject seated opposite observes the finger. The subject's own hand is shielded from the subject's view by a cover. Sensor data from the sensor finger is converted into electrical stimulation patterns applied to the subject's forearm via a medical stimulator and four stimulation electrodes.

in appendix $\mathrm{A}$.

\subsubsection{Participants and experimental conditions}

A total number of 31 healthy, able-bodied volunteers (mean age 28 years, twelve female) participated in this experiment after signing informed consent. Each participant was randomly associated to one of two groups, test group (TEST) or control group (CONTROL). Each group experienced different feedback conditions during the experiment. 


\section{ELECTROCUTANEOUS FEEDBACK TO FACILITATE EMBODIMENT OF PROSTHETIC DEVICES}

Test group A total of 21 subjects were associated to the test group. Spatial information on the interaction locations on the artificial finger, as well as strength of the interaction (light or strong touch) were included in the stimuli in order to provide rich feedback for this group. For this purpose, findings of the first study (cf. chapter 2) are directly employed for designing the feedback provided to the subjects. Firstly, the primary, more obvious aspect of the interaction with the finger, i.e., the location on the finger tip where the skin is touched, was conveyed via spatial encoding, which was found to be most intuitive for subjects in the study investigating different encoding paradigms. Though, strictly-speaking, this spatial encoding of location information is not modality-matched feedback, as the modality of the sensation itself is still changed, the aspect of conveying location information spatially was expected to further assist in the intuitiveness of the feedback.

Secondly, as discussed in chapter 2, the subjects' capability of exploiting highresolution intensity-encoded feedback after a certain accommodation and training phase was taken into account, and a second aspect of the sensory feedback available from the finger, i.e. information on strength of touch, was encoded using intensity modulation on the same electrodes used for the spatial feedback.

Each of the sensors of the artificial finger was allocated to at least one of four areas on the finger tip's surface: front (area 1), back (area 2), left (area 3) and right (area 4). A schematic drawing of the sensor positions inside the artificial finger and the tactile areas contributing to each electrode's stimulation is shown in figure 5.4. Each area was mapped to one of the electrodes on the forearm. The stimulation received on the $i$-th electrode was proportional to the weighted sum $S_{i}$ of the respective activities $s_{n}$ of all sensors in the corresponding area:

$$
S_{i}=\sum_{n \in N_{i}} w_{i, n} \cdot s_{n}
$$

The weights $w$ were chosen to satisfy two conditions. First, the sum of all sensor weights in an area equals one:

$$
\sum_{n \in N_{i}} w_{i, n}=1
$$

Second, as compared to the weights of sensors fully contributing to an area, the weights of sensors allocated to more than one area were reduced by a factor which reflects the number of areas they are associated to. That is, for a sensor, which contributed to the measured activity of two areas, its weight was chosen to be half of the weight of a sensor exclusively associated to that area.

For the interested readers, the following example illustrates the calculation of weights for sensors associated to area 1. Sensors marked 1 and 7-11 contribute 


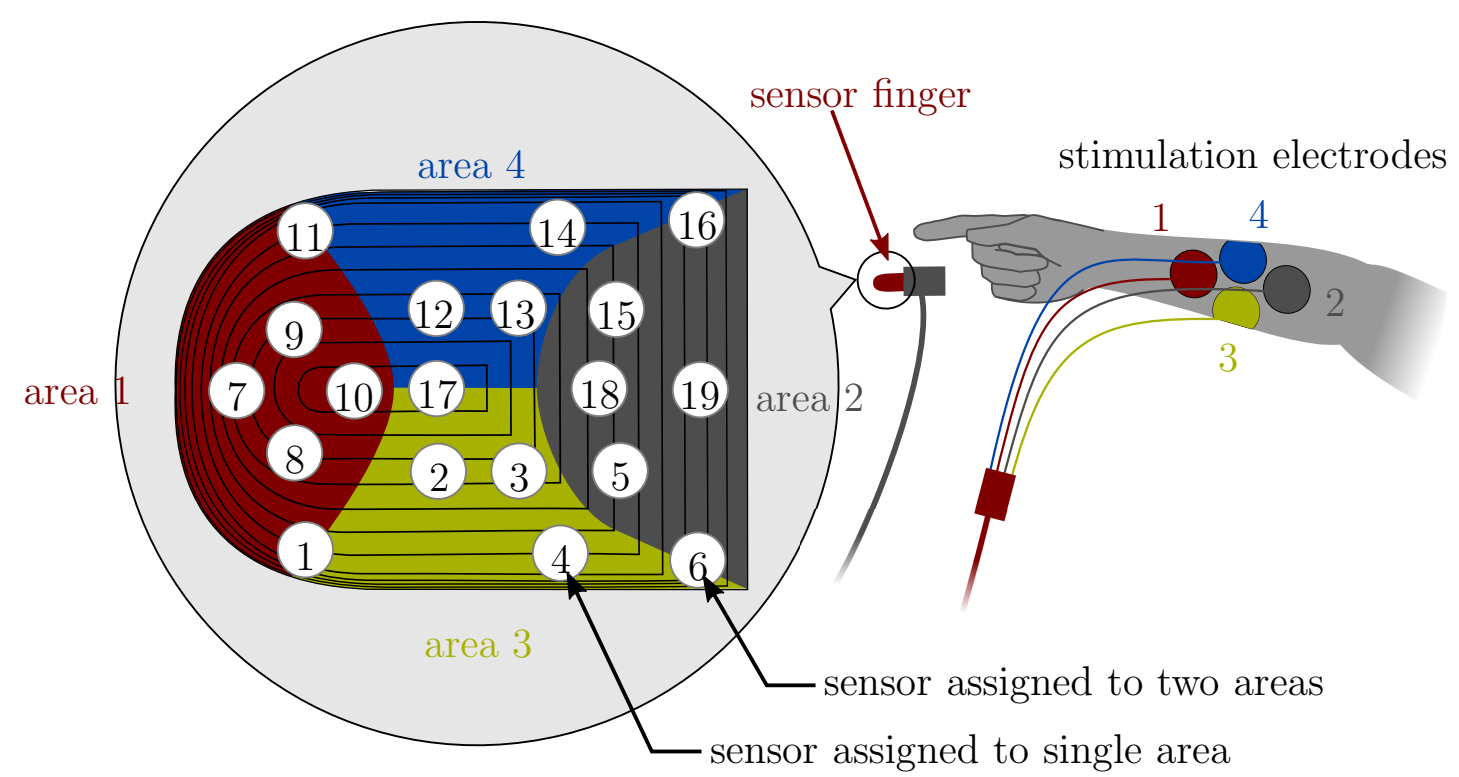

Figure 5.4: Location and indices of sensors inside the artificial finger tip. Each sensor is associated to one or two of four areas, which are mapped to four stimulation sites.

to the overall activity in area 1. According to the second condition, it holds that for $k \in\{1,11\}$ (partially contributing sensors) and $l \in\{7,8,9,10\}$ (fully contributing)

$$
w_{1, k}=0.5 \cdot w_{1, l} \text {. }
$$

From equations 5.2 , where $N_{1}=\{1,7,8,9,10,11\}$, and 5.3 we get that

$$
2 \cdot w_{1, k}+4 \cdot w_{1, l}=5 \cdot w_{1, l}=1
$$

and it follows that for area 1 the weight $w_{1, k}=0.1$ for partially contributing sensors, and $w_{1, l}=0.2$ for fully contributing sensors.

Control group The second group of subjects participating in the experiment was designed as a control group to determine the general effect of non-causal electrocutaneous stimulation, and for comparison of the embodiment effect with the test group.

It has been established, that for the integration of a rubber hand into the self image during rubber hand illusion experiments, the visual and tactile stimuli have to occur simultaneously [61]. Introducing asynchrony has an adverse effect on the development of sense of ownership of the alien hand. The maximum acceptable 


\section{ELECTROCUTANEOUS FEEDBACK TO FACILITATE EMBODIMENT OF PROSTHETIC DEVICES}

delay of $200 \mathrm{~ms}$ to $300 \mathrm{~ms}$, which should not be exceeded, has been determined experimentally in delayed rubber hand illusion experiments by Shimada et al. [67], and recently in the robot hand illusion experiment by Ismail and Shimada [68]. Based on these findings, the stimuli for the control group were designed. Subjects in this group also observed the investigator's interaction with the artificial finger and meanwhile received electrocutaneous stimulation. The stimulation patterns, however, were neither temporally nor spatially congruent with the interactions at the artificial finger. Firstly, while the general feedback was still based on the sensor data, a variable delay was added to the stimulation pattern $(0.5 \mathrm{~s}$ to $1 \mathrm{~s})$. Additionally, in order to introduce further causal decoupling of the stimuli applied to the artificial finger and the electrocutaneous stimulation on the subject's forearm, association between the four sensor areas and the four stimulation areas was randomly switched every three seconds. A total number of ten Subjects were assigned to the control group.

\subsubsection{Outcome measures}

This study uses subjective and objective measures for determining the outcome of the experiment, that is, if integration of the artificial finger into the body scheme occurred.

Proprioceptive drift, which was originally referred to as reach displacement by Botvinick and Cohen [61], is a common measure used in rubber hand illusion experiments. Before and after the intervention, subjects are asked to point to the perceived location of a certain reference point, e.g. the tip of their index finger. As their hand is covered during the experiment, they have to rely on their sense of proprioception. The difference between the estimate made after the intervention and the estimate made before is called proprioceptive drift. A drift towards the rubber hand is associated to the subject attributing the rubber hand to his own body, in contrast to a drift away from the rubber hand. For the original rubber hand illusion experiment, a mean proprioceptive drift of $2.3 \mathrm{~cm}$ towards the rubber hand was reported for the test group, while in the control group, a mean drift of $1.3 \mathrm{~cm}$ away from it occurred [61]. Similar values were found by Tsakiris and Haggard, who observed a mean drift of almost $3 \mathrm{~cm}$ towards, and almost $1 \mathrm{~cm}$ away from the rubber hand in test and control group, respectively. In this study, proprioceptive drift was determined for the index finger tip. It was hypothesised that under the TEST condition, subjects would exhibit positive proprioceptive drift, while in the CONTROL condition no or negative drift was expected.

As an objective measure, the change of temperature of the index finger is employed. In a comprehensive study by Moseley et al. it was found that the body part which is the object of a rubber hand illusion experiments cools down [69]. 
The stronger the perceived illusion is, the more pronounced the temperature drop in the respective body part. It was concluded that the reverse effect of embodiment takes place here, and that while the rubber hand is successfully perceived as part of the body and integrated into the subject's body scheme, the subject's own hand is simultaneously disembodied. This manifests in reduced circulation, which in turn results in decrease of skin temperature.

It was expected to observe a similar effect if the transfer of sensory input from the artificial finger tip to the forearm via electrocutaneous stimulation was successfully facilitating embodiment of the finger.

\subsubsection{Experimental procedure}

In this experiment, a $100 \mathrm{~Hz}$ stimulation with fixed stimulation current of $3 \mathrm{~mA}$ was used. Those subjects unfamiliar with electrocutaneous stimulation were given the opportunity to experience some low intensity stimulation, and feel the effect of different pulse frequencies. After that, the sensation thresholds (i.e., minimum stimulation pulse width necessary to elicit a sensation) and comfort thresholds (i.e., upper limit for stimulation pulse width before stimulation is perceived as uncomfortable) were systematically determined for each of the electrodes. The test environment was configured to map the complete stimulation range (sensation to comfort threshold) to the sensor input range (light touch/deformation of the skin to strong touch/deformation of the skin).

The rubber finger illusion experiment itself was split into three runs. The first and second run lasted five minutes, the last run three minutes. In between runs subjects were given five minute breaks to relax. During each run, the investigator interacted with the artificial finger by using a combination of three different methods: simple touching, stroking and flicking. Touching focused on single point interactions and was aimed at creating pressure on different locations all over the sensor finger. Stroking was either executed from tip to back or vice versa, or from left to right or vice versa. Care was taken to perform a uniform stroke in one direction that would result in a constant pressure point shifting across the finger. Flicking meant flicking the side of the rubber finger. It shall be noted that flicking caused a short sharp burst of activity to be detected by all of the finger's sensors.

Before the first run, subjects were asked to estimate and point to the position of their own fingertip under the cover, and the skin temperature on the subject's fingertip was measured using a non-contact infrared thermometer. This procedure was repeated immediately after each of the three runs. 


\section{ELECTROCUTANEOUS FEEDBACK TO FACILITATE EMBODIMENT OF PROSTHETIC DEVICES}

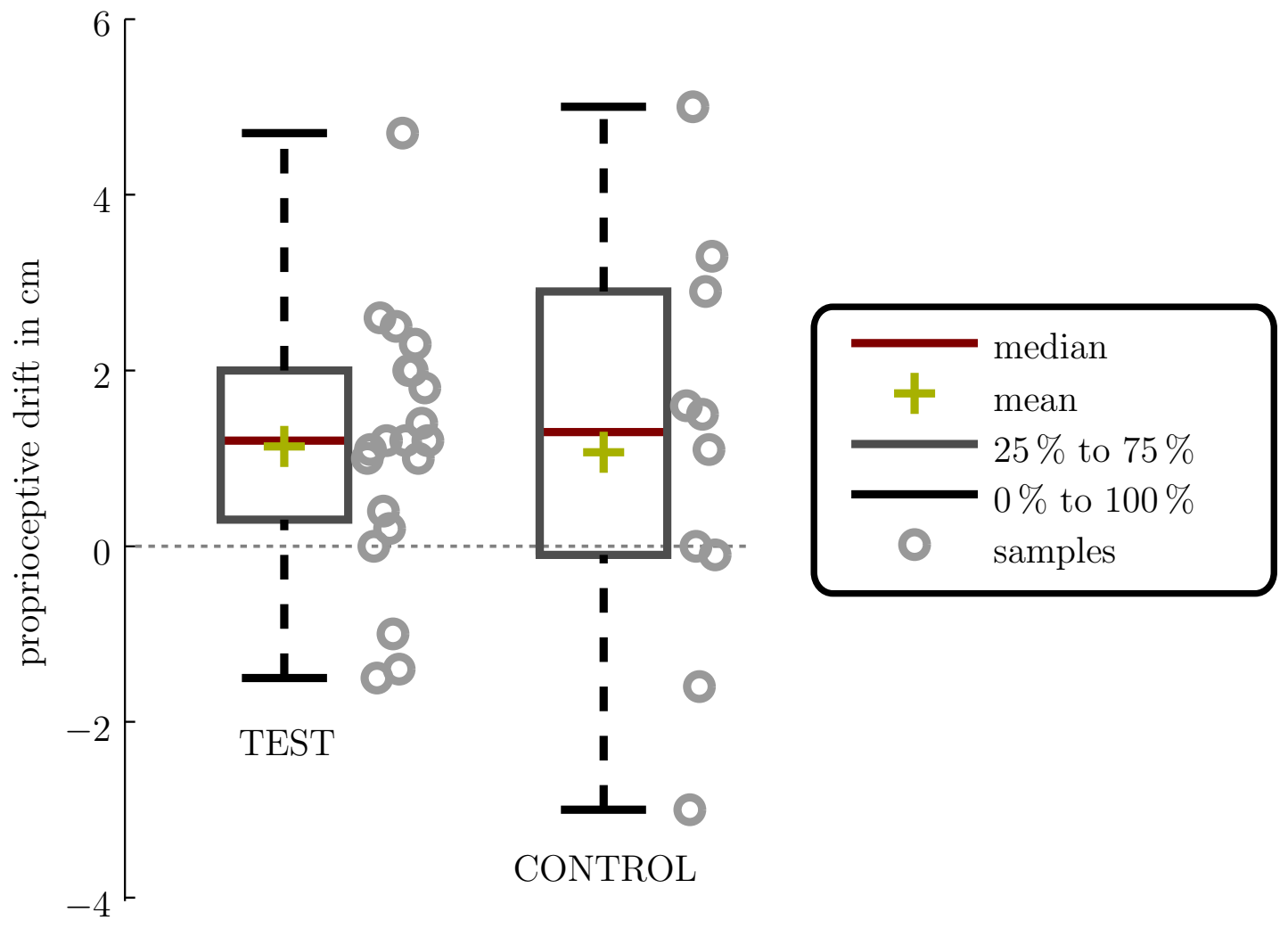

Figure 5.5: Comparison of proprioceptive drift measurements. In both conditions, a slight positive drift towards the artificial finger occured in average (TEST: $1.1 \mathrm{~cm} \pm 2.4 \mathrm{~cm}$, CONTROL: $1.1 \mathrm{~cm} \pm 2.4 \mathrm{~cm}$ ). The was no statistically significant difference $(p>0.9)$.

\subsection{Results}

Figure 5.5 shows the results of all available measures of the total proprioceptive drift observed. For one subject of the test group, proprioceptive drift had to be discarded due to movement of the subject, before the measure could be taken. Under the TEST condition, subjects predominantly expressed positive proprioceptive drift. Only four out of 20 measurements showed no or negative proprioceptive drift. The total range of drift observed was $[-1.5 \mathrm{~cm}, 4.7 \mathrm{~cm}]$, the mean and standard deviation of proprioceptive drift in the test group were $1.1 \mathrm{~cm} \pm 1.5 \mathrm{~cm}$. Very similar results were observed for the CONTROL condition. Also for this group, positive drifts were determined for six out of ten measurements, while four subjects showed no or negative proprioceptive drift. The total range of drift observed was $[-3.0 \mathrm{~cm}, 5.0 \mathrm{~cm}]$, the mean and standard deviation in the con- 
trol group were $1.1 \mathrm{~cm} \pm 2.4 \mathrm{~cm}$. No statistical difference between the conditions TEST and CONTROL was found $(p>0.9)$.

Figure 5.6 shows the results of the temperature measurement. In the test group, finger temperature changes were generally measured around $0{ }^{\circ} \mathrm{C}$, while for the control group they were predominantly positive. In the test group, about half of the subjects showed a drop in finger temperature, while for the other half a rise in temperature was detected. In average, the finger temperature decreased by $0.19^{\circ} \mathrm{C} \mp 1.88^{\circ} \mathrm{C}$ during the experiment. In the control group positive changes were measured in all subjects except one. In average, the finger temperature increased by $1.81{ }^{\circ} \mathrm{C} \pm 2.06^{\circ} \mathrm{C}$ for subjects in the control group. The difference between groups was statistically significant $(p<0.05)$.

\subsection{Discussion}

The literature often reports differences in the mean proprioceptive drift for treatment and control groups $[61,64,66,70]$. Interestingly, the current study was not able to reproduce this effect, and no notable difference between the treatment and control group could be measured $(p>0.9)$. However, the range of drifts observed here $(-1.5 \mathrm{~cm}$ to $4.7 \mathrm{~cm})$ is similar to the range of drifts observed by Botvinik and Cohen (ca. $-4 \mathrm{~cm}$ to $9 \mathrm{~cm}$ ) [61], especially when taking into account that also the initial distance between the artificial real limb was larger in that study. Unfortunately, this statement can only be made for the test condition as presented in the original rubber hand illusion paper, as individual results for the control condition were not reported, and other studies likewise only report mean values.

Thus said, the main difference found as compared to studies reported in the literature was, that the majority of subjects experienced comparable proprioceptive drift towards the artificial finger, regardless of the feedback condition (TEST, CONTROL). Only few subjects estimated their finger to have the same distance or be farther away from the sensor finger after the experiment as compared to before the experiment. These results seem to be more in line with findings by Rosén et al., who reported that only three out of five amputees showed greater positive proprioceptive drift in a robot hand illusion experiment during the test condition (synchronous stimuli), as compared to the control condition (asynchronous stimuli) [62].

A comprehensive study by Tsakiris and Haggard [70] offers a possible explanation as to why this could be the case. They investigated if proprioceptive drift was different for a finger that was not the object of the illusion experiment (i.e., did not receive stimuli), as compared to the drift of the finger that had been stimulated during the intervention (stroking with a brush). In fact, results of the test 


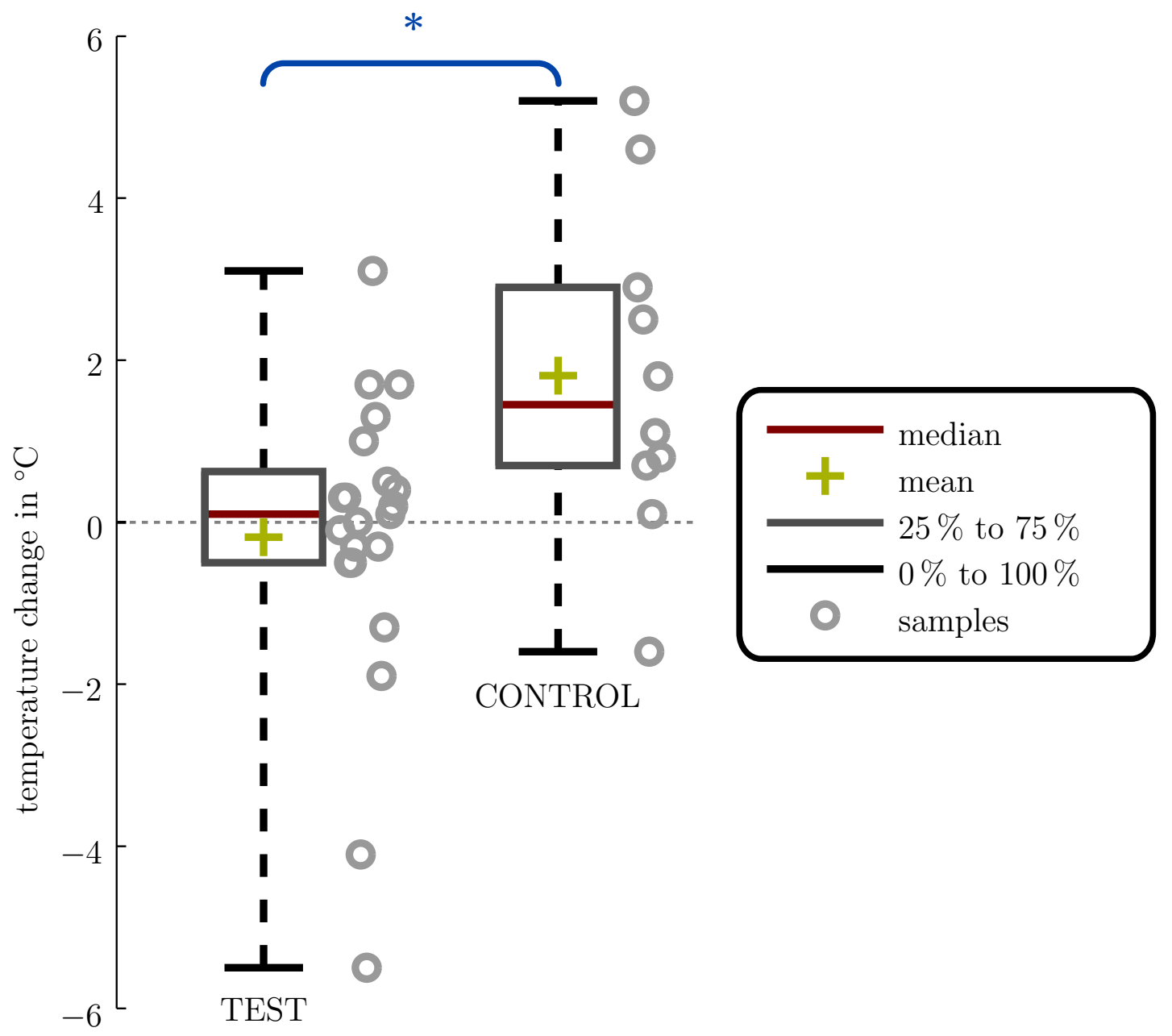

Figure 5.6: Comparison of change in finger temperature across groups. For the test group, the finger temperature slightly decreased during the experiment $\left(-0.19^{\circ} \mathrm{C} \pm 1.88^{\circ} \mathrm{C}\right)$, while for the control group, finger temperature generally increased $\left(1.81^{\circ} \mathrm{C} \pm 2.06^{\circ} \mathrm{C}\right)$. The difference was statistically significant $(p<0.05)$. 
and control conditions were only found to be significantly different, if the subjects had to estimate the position of the same finger that was stroked. When position was estimated for a different finger, no significant differences were found.

This finding could rely to findings of the current study, as also here the position estimate was made for a finger that did not actually receive any stimulation during the experiment. Hence, it is assumed, that the chosen measure of proprioceptive drift of the finger may not be a reliable measurement for embodiment, when the artificial feedback is actually supplied to the forearm.

Results for the temperature measurement were more conclusive. Based on the findings by Moseley et al. [69], a slight decrease in skin temperature was expected for the test group, while skin temperature for control group subjects was expected to remain unchanged. Contrary to that, while a very slight average decrease was found for the test group $\left(-0.19^{\circ} \mathrm{C} \pm 1.88^{\circ} \mathrm{C}\right)$, the participants of the control group showed a notable average temperature increase $\left(1.81^{\circ} \mathrm{C} \pm 2.06^{\circ} \mathrm{C}\right)$. Two explanations for this phenomenon are possible. The first would be that a systematic error in the setup or measurement was the cause. Firstly, changes in the room temperature were possible (no climate control available), and may have influenced the measurement. The second explanation is that warming of the hand is an effect unrelated to environmental conditions, and that it was directly caused by the application of electrocutaneous stimulation, which is known to enhance circulation. In both cases, the effects should be visible for both TEST and CONTROL condition, which still makes it possible to draw conclusions from the measurement of the skin temperature.

As changes in skin temperature showed a positive offset with respect to the initial expectations for both groups, it is assumed that general offset was added to the temperature development. This can be a result either of environmental conditions influencing the measurement, or of experimental procedures (i.e., increased circulation due to electrocutaneous stimulation). As a consequence, this offset is presumed to have compensated, for instance, an actual cooling effect in the test group, and to have shifted measurements for the control group away from the expected zero line. Following these assumptions and from the significantly different temperature development observed in the test and control groups, it can be concluded that embodiment of an artificial finger could actually be induced by providing sensory substitution feedback via electrocutaneous stimulation on the forearm. This is an exciting result, with interesting implications for amputees and the possibilities to provide sensory feedback that aids them in incorporating their prostheses into their body scheme. Further psychological and medical benefits are expected, such as increased acceptance of prosthetic devices, reversal of maladaptive plasticity, and alleviation of phantom limb pain [7]. 
5. ELECTROCUTANEOUS FEEDBACK TO FACILITATE EMBODIMENT OF PROSTHETIC DEVICES 


\section{Chapter 6}

\section{Summary \& Conclusions}

Loss of a hand has severe implications for the daily life of affected people. Though modern upper limb prosthetics offer some relief, as they can restore rudimentary motor function, amputees are still deprived of the rich and abundant sensory feedback naturally available from the hand. This dissertation is dedicated to the application of electrocutaneous stimulation as means for providing sensory feedback to amputees. Particular focus is on investigating its feasibility in combination with myoelectric prosthesis control, potential benefits arising from the resulting new possibility for closed-loop prosthesis control, and the feedback's role in facilitating embodiment of prosthetic components. For these purposes, a series of four independent studies was conducted.

First, different approaches for encoding feedback information into electrocutaneous stimulation patterns were proposed and evaluated. Although a pure spatial representation of the feedback variable by means of utilising a number of stimulation sites seemed intuitive, its major disadvantage proved to be the need for digitising the feedback variable, which leads to a loss in information resolution. This became apparent, when comparing this approach to pure intensity encoding, i.e., the information variable is presented via a single stimulation channel, where the range of the feedback information is mapped to a range of comfortable and perceivable stimulation intensities. Here, high resolution of information can be maintained. However, slow changes in stimulation intensity are hard to perceive and may remain unnoticed. An approach uniting advantages of both these methods is a combined spatial/intensity approach. Since results for pure intensity encoding were good, however, for practical reasons (spatial restrictions for integration of stimulation electrodes in real-life prostheses), it is recommended to focus on pure intensity feedback.

Spatial restrictions for placement of electrodes are indeed an issue, that is even likely to become more pressing in the near future, when more and more electrodes need to be positioned within the socket for more advanced control of increasingly 


\section{SUMMARY \& CONCLUSIONS}

complex and dexterous prosthetic systems. Arising from the close proximity of stimulation and recording electrodes is the issue of interference on the EMG caused by nearby electrocutaneous stimulation. This issue was thoroughly addressed in this work, and it was shown that electrocutaneous stimulation is still viable, despite its influence on the quality of the EMG signal. A practical approach, namely artefact blanking, has been evaluated in the context of both classic proportional control, as well as pattern recognition applied to multi-channel EMG data. It was shown to be effective in restoring benchmark performance of both control approaches as determined without any electrical stimulation. Stimulation pulse widths of up to $400 \mu \mathrm{s}$ in combination with an artefact occurrence frequency of up to $150 \mathrm{~Hz}$ could be handled without loss of control accuracy.

For on-line evaluation of its performance with direct proportional prosthesis control, a low-cost implementation of the blanking approach was chosen. This approach uses signal mirroring to provide compensatory data samples and allow a fixed window length implementation. This limits the portion of signal that can at most be contaminated by artefacts to $50 \%$, which was shown to be sufficient in the practical evaluation. In case of larger portions of the signal being unusable, the dynamic segmentation approach evaluated for pattern recognition can be applied without expectation of performance loss. In fact, using root mean square value of EMG to estimate muscle activity for direct proportional control corresponds to one of the feature sets investigated in the classification scenario. Additionally, with the conclusions that could be drawn from the analysis of the stimulation artefacts themselves, the following recommendations are made in order to limit the manifestation and with it impact of the stimulation artefacts in the first place: Increase in stimulation current has far less effect on the amplitude and duration of stimulation artefacts, than increase of pulse width. Therefore, stimulation current is the preferable variable of choice to increase if perceived intensity of the stimulation shall be increased. In doing so, however, it must be noted, that care must be taken not to exceed certain thresholds for the current density, as this can be harmful for the skin. As a consequence, stimulation electrode area must be taken into account when determining safe limits of stimulation current, as the use of smaller electrodes will result in higher current densities at the same absolute current. However, higher current densities elicit stronger sensations, which is why in general lower currents suffice to achieve with small electrodes the same effect as with larger electrodes. Only as a second choice, should pulse width be increased, e.g. when stimulation current has reached its safe limit, and the perceived sensation is deemed to weak by the user. In general, pulse width can be increased until approximately $40 \%$ of the signal are rendered unusable due to artefact contamination. Only beyond that, losses in control accuracy were shown to occur.

The feedback encodings used in subsequent studies were designed to take into ac- 
count both the findings of the first study, and the second study. Hence, feedback could be ensured to be well perceivable, and stimulation parameters were in a range so that the issue of stimulation artefacts was easily controllable.

Having established that, it was investigated in how far sensory feedback can be beneficial for prostheses users in a sense that it either improves their control accuracy or speeds up their mastering the use of the prosthesis. An experiment was designed to emulate learning of routine grasping. Routine grasping is a fast activity, where grabbing and lifting an object takes place within the fraction of a second. This does not allow for extensive steering and modulation of the control signal, or active contemplation of sensory feedback. Instead, it was hypothesised that sensory feedback could facilitate learning on a trial to trial basis, where information obtained from the previous grasp attempt is taken into account when preparing the following one. However, results of the study clearly showed that although subjects were able to improve with time, there was no significant difference between subjects who only received basic feedback on the success or failure of the grasp attempts, as compared to those receiving information on the currently exerted grasp force via a tactile or visual channel. Though a trend was visible for slightly lower force variability in the group receiving tactile feedback, all subjects were able to significantly reduce force variability and improve grasp success. From this, it can be concluded that the very basic feedback available in any case (observation of prosthesis and outcome of grasp attempt), is sufficient for mastering a routine grasping task. Additional sensory feedback does not add functional advantages for this specific scenario, but, importantly, it does not have a detrimental effect either. This is an important finding related to the application of sensory feedback for non-functional purposes.

For instance, it is an established benefit of both sensory feedback as well as electrocutanous stimulation that it alleviates phantom limb pain $[7,60]$. Associated to that, it is hypothesised that the ability of an amputee to integrate a prosthetic component into his body scheme has a positive effect on reversing maladaptive plastic changes in the cortex, which are believed to be the cause for phantom limb pain [71]. It is assumed that providing sensory feedback aids this process of embodiment. A study was presented investigating if providing artificial sensory feedback from a prosthetic component via electrocutanous stimulation at a site not previously associated with the body part being replaced, can facilitate embodiment of said prosthetic component. Specifically, electrocutanous stimulation on the forearm was used to transmit touch information from the distal phalanx of an artificial finger. Findings from the first study were employed to design a feedback which was both intuitive and capable of transmitting high-resolution information. Results were ambiguous with regard to two outcome measures, where one supported the approach, and the other remained indifferent. Different development of skin temperature in test and control group confirmed that embodiment 


\section{SUMMARY \& CONCLUSIONS}

was achieved. In fact, a temperature offset with respect to the expected changes in temperature was found to reduce the expected cooling of the finger. However, since this offset was present for both control and test group, it was assumed that either conditions, or indeed enhanced circulation due to electrocutaneous stimulation introduced the temperature offset.

Regarding the expected proprioceptive drift, no differences were found between the conditions. Members of the control group showed the same evidence of misjudging the position of their own finger, as members of the test group. This result is not congruent with the results obtained from similar experiments, where significant differences in this measure are usually observed between test and control groups. However, work by Tsakiris and Haggard [70] suggests that proprioceptive drift only delivers significantly different results for test and control group if based on the finger that has received stimuli during the experiment. As in this study the forearm was stimulated, this could be a reason why estimation of finger position did not result in different results for test and control group.

Many questions with respect to further possible benefits of sensory feedback in general were left unaddressed and pose interesting directions for future research. At that, electrocutaneous stimulation as means to provide said feedback should be taken into account. In conclusion it was shown that electrocutaneous feedback is a viable approach to provide sensory feedback to amputees. Though not necessary for mastering a routine grasping task, evidence points to its usefulness for embodiment of prosthetic components.

Striving for innovative and demonstrable solutions that further improve the life of amputees and seek to alleviate their loss remains a goal, that through this work has become more achievable. It was shown that electrocutaneous feedback can be exploited by humans in challenging control tasks, and that it plays a significant role in the embodiment of prosthetic devices. Furthermore, the work presented in this dissertation has established beyond doubt that any detrimental effects of stimulation artefacts on prosthesis control can be handled by a practical blanking and segmentation approach. These methods are now available and allow combination of electrocutaneous stimulation with myocontrol while still ensuring optimal control performance and self containment of the prosthesis. This provides a solid foundation for further application of electrocutaneous stimulation feedback, and allows to finally pursue its integration into commercial devices, making the technology and its benefits available to amputees. 


\section{Bibliography}

[1] R. J. van Beers, A. C. Sittig, and J. a. J. D. van der Gon, "Integration of proprioceptive and visual position-information: An experimentally supported model.," Journal of neurophysiology, vol. 81, no. 3, pp. 1355-64, 1999. 1

[2] S. J. Sober and P. N. Sabes, "Multisensory integration during motor planning.," The Journal of neuroscience : the official journal of the Society for Neuroscience, vol. 23, no. 18, pp. 6982-6992, 2003. 1

[3] M. C. Dadarlat, J. E. O'Doherty, and P. N. Sabes, "A learning-based approach to artificial sensory feedback leads to optimal integration," Nature Neuroscience, vol. 18, no. 1, pp. 138-144, 2014. 1, 9, 72

[4] E. Biddiss, D. Beaton, and T. Chau, "Consumer design priorities for upper limb prosthetics," Disability and Rehabilitation: Assistive Technology, vol. 2, no. 6, pp. 346-357, 2007. 2

[5] C. Pylatiuk, S. Schulz, and L. Döderlein, "Results of an Internet survey of myoelectric prosthetic hand users," Prosthetics and orthotics international, vol. 31, no. 4, pp. 362-370, 2007. 2

[6] J. D. Brown, A. Paek, M. Syed, M. K. O’Malley, P. A. Shewokis, J. L. Contreras-Vidal, A. J. Davis, and R. B. Gillespie, "Understanding the Role of Haptic Feedback in a Teleoperated/Prosthetic Grasp and Lift Task," in 2013 World Haptics Conference (WHC), pp. 271-276, apr 2013. 2

[7] C. Dietrich, K. Walter-Walsh, S. Preißler, G. O. Hofmann, O. W. Witte, W. H. R. Miltner, and T. Weiss, "Sensory feedback prosthesis reduces phantom limb pain: Proof of a principle," Neuroscience Letters, vol. 507, no. 2, pp. $97-100,2012.2,83,87$

[8] H. Flor, "Phantom-limb pain: characteristics, causes, and treatment," The Lancet Neurology, vol. 1, pp. 182-189, jul 2002. 2 
[9] X. Wang, L. Dong, H. Zhang, R. Yu, C. Pan, and Z. L. Wang, "Recent Progress in Electronic Skin," Advanced Science, vol. 2, no. 10, p. 1500169 , 2015. 2

[10] P. D. Marasco, K. Kim, J. E. Colgate, M. A. Peshkin, and T. A. Kuiken, "Robotic touch shifts perception of embodiment to a prosthesis in targeted reinnervation amputees," Brain, vol. 134, no. 3, pp. 747-758, 2011. 3

[11] R. E. Prior and J. Lyman, "Electrocutaneous feedback for artificial limbs. Summary progress report. February 1, 1974, through July 31, 1975," Bulletin of prosthetics research, no. 10-24, pp. 3-37, 1975. 3, 4

[12] R. E. Prior, J. Lyman, P. A. Case, and C. M. Scott, "Supplemental sensory feedback for the VA/NU myoelectric hand. Background and preliminary designs.," Bulletin of prosthetics research, vol. 101, no. 134, pp. 170-191, 1976. 3,6

[13] G. F. Shannon, "A myoelectrically-controlled prosthesis with sensory feedback.," Medical \& biological engineering \&6 computing, vol. 17, no. 1, pp. 7380, 1979. 3

[14] R. N. Scott, R. R. Caldwell, R. H. Brittain, A. B. Cameron, and V. A. Dunfield, "Sensory-feedback system compatible with myoelectric control," Medical and Biological Engineering and Computing, vol. 18, no. 1, pp. 6569, 1980. 3

[15] S. G. Meek, S. C. Jacobsen, and P. P. Goulding, "Extended physiologic taction: design and evaluation of a proportional force feedback system.," Journal of rehabilitation research and development, vol. 26, no. 3, pp. 53-62, 1989. 3

[16] C. Antfolk, M. D’Alonzo, B. Rosén, G. Lundborg, F. Sebelius, and C. Cipriani, "Sensory feedback in upper limb prosthetics.," Expert review of medical devices, vol. 10, no. 1, pp. 45-54, 2013. 3

[17] P. E. Patterson and J. A. Katz, "Design and Evaluation of a Sensory Feedback System that Provides Grasping Pressure in a Myoelectric Hand," Journal of Rehabilitation Research and Development, vol. 29, no. 1, pp. 1-8, 1992. 3

[18] M. C. Jimenez and J. A. Fishel, "Evaluation of Force, Vibration and Thermal Tactile Feedback in Prosthetic Limbs," 2014 Ieee Haptics Symposium (Haptics), pp. 437-441, 2014. 3 
[19] A. Y. J. Szeto and F. A. Saunders, "Electrocutaneous Stimulation for Sensory Communication in Rehabilitation Engineering," IEEE Transactions on Biomedical Engineering, vol. BME-29, no. 4, pp. 300-308, 1982. 4, 35

[20] M. Solomonow and J. Lyman, "Electrotactile stimulation relevant to sensorymotor rehabilitation: a progress report." Bulletin of prosthetics research, vol. 17 , no. 1 , pp. $63-72,1980.4,35$

[21] G. Chai, X. Sui, S. Li, L. He, and N. Lan, "Characterization of evoked tactile sensation in forearm amputees with transcutaneous electrical nerve stimulation," Journal of Neural Engineering, vol. 12, no. 6, p. 066002, 2015. 4

[22] R. R. Riso, "Strategies for providing upper extremity amputees with tactile and hand position feedback-moving closer to the bionic arm.," Technology and health care : official journal of the European Society for Engineering and Medicine, vol. 7, no. 6, pp. 401-409, 1999. 4

[23] X. Navarro, T. B. Krueger, N. Lago, S. Micera, T. Stieglitz, and P. Dario, "A Critical Review of Interfaces with the Peripheral Nervous System for the Control of Neuroprotheses and Hybrid Bionic Systems," J Peripher Nerv Syst, vol. 10, pp. 229-258, 2005. 4

[24] G. S. Dhillon and K. W. Horch, "Direct neural sensory feedback and control of a prosthetic arm," IEEE Transactions on Neural Systems and Rehabilitation Engineering, vol. 13, no. 4, pp. 468-472, 2005. 4

[25] K. Horch, S. Meek, T. G. Taylor, and D. T. Hutchinson, "Object discrimination with an artificial hand using electrical stimulation of peripheral tactile and proprioceptive pathways with intrafascicular electrodes," IEEE Transactions on Neural Systems and Rehabilitation Engineering, vol. 19, no. 5, pp. $483-489,2011.4$

[26] T. Stieglitz, T. Boretius, X. Navarro, J. Badia, D. Guiraud, J. L. Divoux, S. Micera, P. M. Rossini, K. Yoshida, K. R. Harreby, A. Kundu, and W. Jensen, "Development of a neurotechnological system for relieving phantom limb pain using transverse intrafascicular electrodes (TIME)," Biomedizinische Technik, vol. 57, no. 6, pp. 457-465, 2012. 4

[27] S. Raspopovic, M. Capogrosso, F. M. Petrini, M. Bonizzato, J. Rigosa, G. Di Pino, J. Carpaneto, M. Controzzi, T. Boretius, E. Fernandez, G. Granata, C. M. Oddo, L. Citi, A. L. Ciancio, C. Cipriani, M. C. Carrozza, W. Jensen, 
E. Guglielmelli, T. Stieglitz, P. M. Rossini, and S. Micera, "Restoring Natural Sensory Feedback in Real-Time Bidirectional Hand Prostheses," Science Translational Medicine, vol. 6, p. 222ra19, feb 2014. 4

[28] G. Di Pino, A. Benvenuto, M. Tombini, G. Cavallo, L. Denaro, V. Denaro, F. Ferreri, L. Rossini, S. Micera, E. Guglielmelli, and P. M. Rossini, "Overview of the implant of intraneural multielectrodes in human for controlling a 5-fingered hand prosthesis, delivering sensorial feedback and producing rehabilitative neuroplasticity," Proceedings of the IEEE RAS and EMBS International Conference on Biomedical Robotics and Biomechatronics, pp. 1831-1836, 2012. 4

[29] S. Dosen, M. Markovic, C. Hartmann, and D. Farina, "Sensory Feedback in Prosthetics: A Standardized Test Bench for Closed-Loop Control.," IEEE Transactions on Neural Systems and Rehabilitation Engineering, vol. 23, pp. 267-276, mar 2015. 10, 16, 17, 18, 19, 20, 97

[30] A. Y. J. Szeto and J. Lyman, "Comparison of codes for sensory feedback using electrocutaneous tracking.," Annals of biomedical engineering, vol. 5, pp. 367-383, 1977. 10, 11, 22

[31] L. P. Paredes, S. Dosen, F. Rattay, B. Graimann, and D. Farina, "The impact of the stimulation frequency on closed-loop control with electrotactile feedback," Journal of NeuroEngineering and Rehabilitation, vol. 12, no. 1, pp. 1-16, 2015. 11, 22

[32] F. A. A. Kingdom and N. Prins, Psychophysics: A Practical Introduction. Academic Press, 2nd ed., 2016. 13

[33] A. Y. J. Szeto, J. Lyman, and R. E. Prior, "Electrocutaneous Pulse Rate and Pulse Width Psychometric Functions for Sensory Communications," Human Factors: The Journal of the Human Factors and Ergonomics Society, vol. 21, no. 2, pp. 241-249, 1979. 22

[34] D. S. Childress, "Powered Limb Prostheses: Their Clinical Significance," IEEE Transactions on Biomedical Engineering, vol. BME-20, no. 3, pp. 200207, 1973. 25

[35] C. Hartmann, S. Došen, S. Amsuess, and D. Farina, "Closed-loop control of myoelectric prostheses with electrotactile feedback: Influence of stimulation artifact and blanking," IEEE Transactions on Neural Systems and Rehabilitation Engineering, vol. 23, no. 5, pp. 807-816, 2015. 25, 28, 30, 31, 33, 34, $37,38,39,41,42,43$ 
[36] F. Mandrile, D. Farina, M. Pozzo, and R. Merletti, "Stimulation Artifact in Surface EMG Signal: Effect of the Stimulation Waveform, Detection System, and Current Amplitude Using Hybrid Stimulation Technique," IEEE Transactions on Neural Systems and Rehabilitation Engineering, vol. 11, no. 4, pp. 407-415, 2003. 26

[37] C. Almström, A. Anani, P. Herberts, and L. Körner, "Electrical stimulation and myoelectric control. A theoretical and applied study relevant to prosthesis sensory feedback.," Medical \& Biological Engineering \&f Computing, vol. 19, pp. 645-653, sep 1981. 27

[38] C. Frigo, M. Ferrarin, W. Frasson, E. Pavan, and R. Thorsen, "EMG signals detection and processing for on-line control of functional electrical stimulation.," Journal of Electromyography and Kinesiology, vol. 10, no. 5, pp. 351360, 2000. 27

[39] H. Asanuma and I. Rosén, "Spread of mono- and polysynaptic connections within cat's motor cortex," Experimental Brain Research, vol. 16, no. 5, pp. 507-520, 1973. 27

[40] X. Yi, J. Jia, S. Deng, S. G. Shen, Q. Xie, and G. Wang, "A Blink Restoration System With Contralateral EMG Triggered Stimulation and Real-Time Artifact Blanking," IEEE Transactions on Biomedical Circuits and Systems, vol. 7 , no. 2 , pp. 140-148, 2013. 27

[41] T. Keller and M. R. Popovic, "Real-time stimulation artifact removal in EMG signals for neuroprosthesis control applications.," Proceedings of the 6th Annual IFESS Conference, pp. 4-6, 2001. 27

[42] T. Schauer, R. Salbert, N.-O. Negard, and J. Raisch, "Detection and Filtering of EMG For Assessing Voluntary Muscle Activity During FES," in 9th Annual Conference of the International FES Society, (Bournemouth), pp. 185-187, sep 2004. 27

[43] R. B. Pilkar, M. Yarossi, and G. Forrest, "Empirical mode decomposition as a tool to remove the function Electrical stimulation artifact from surface electromyograms: Preliminary investigation," Proceedings of the Annual International Conference of the IEEE Engineering in Medicine and Biology Society, EMBS, pp. 1847-1850, 2012. 27

[44] A. H. Arieta, H. Yokoi, T. Arai, and W. Yu, "Study on the Effects of Electrical Stimulation on the Pattern Recognition for an EMG Prosthetic Application," in 2005 IEEE Engineering in Medicine and Biology 27th Annual Conference, pp. 6919-6922, jan 2005. 27 
[45] M. Asghari Oskoei and H. Hu, "Myoelectric control systems - A survey," Biomedical Signal Processing and Control, vol. 2, no. 4, pp. 275-294, 2007. 28

[46] K. Englehart and B. Hudgins, "A robust, real-time control scheme for multifunction myoelectric control," IEEE Transactions on Bio-Medical Engineering, vol. 50, no. 7, pp. 848-54, 2003. 29, 50, 55

[47] E. J. Scheme, K. B. Englehart, and B. S. Hudgins, "Selective classification for improved robustness of myoelectric control under nonideal conditions," IEEE Transactions on Biomedical Engineering, vol. 58, no. 6, pp. 1698-1705, 2011. 29,55

[48] B. Peerdeman, D. Boere, H. Witteveen, R. Huis in 'tVeld, H. Hermens, S. Stramigioli, H. Rietman, P. Veltink, and S. Misra, "Myoelectric forearm prostheses: State of the art from a user-centered perspective," The Journal of Rehabilitation Research and Development, vol. 48, no. 6, p. 719, 2011. 29

[49] M. Zardoshti-Kermani, B. C. Wheeler, K. Badie, and R. M. Hashemi, "EMG feature evaluation for movement control of upper extremity prostheses," IEEE Transactions on Rehabilitation Engineering, vol. 3, no. 4, pp. 324333, 1995. 29

[50] M. Zecca, S. Micera, M. C. Carrozza, and P. Dario, "Control of multifunctional prosthetic hands by processing the electromyographic signal.," Critical reviews in biomedical engineering, vol. 30, no. 4-6, pp. 459-85, 2002. 29

[51] A. V. Oppenheim, R. W. Schafer, and J. R. Buck, "Discrete Time Signal Processing," 1999. 29

[52] S. Dosen, M. Markovic, K. Somer, B. Graimann, and D. Farina, "EMG Biofeedback for online predictive control of grasping force in a myoelectric prosthesis.," Journal of neuroengineering and rehabilitation, vol. 12, no. 1, p. $55,2015.48,69$

[53] L. H. Smith, L. J. Hargrove, B. A. Lock, and T. A. Kuiken, "Determining the optimal window length for pattern recognition-based myoelectric control: Balancing the competing effects of classification error and controller delay," IEEE Transactions on Neural Systems and Rehabilitation Engineering, vol. 19, no. 2, pp. 186-192, 2011. 50, 55

[54] L. Liu, P. Liu, E. A. Clancy, E. Scheme, and K. B. Englehart, "Electromyogram whitening for improved classification accuracy in upper limb prosthesis 
control," IEEE Transactions on Neural Systems and Rehabilitation Engineering, vol. 21, no. 5, pp. 767-774, 2013. 50

[55] B. Hudgins, P. Parker, S. Member, N. Robert, and S. Member, "A New Strategy for Multifunction Myoelectric Control," IEEE Transactions on Biomedical Engineering, vol. 40, no. I, pp. 82-94, 1993. 55

[56] M. Desmurget and S. Grafton, "Forward modeling allows feedback control for fast reaching movements," Trends in Cognitive Sciences, vol. 4, no. 11, pp. $423-431,2000.56$

[57] S. Dosen, M. Markovic, N. Wille, M. Henkel, M. Koppe, A. Ninu, C. Frömmel, and D. Farina, "Building an internal model of a myoelectric prosthesis via closed-loop control for consistent and routine grasping," Experimental Brain Research, vol. 233, no. 6, pp. 1855-1865, 2015. 56

[58] A. Ninu, S. Dosen, S. Muceli, F. Rattay, H. Dietl, and D. Farina, "Closedloop control of grasping with a myoelectric hand prosthesis: Which are the relevant feedback variables for force control?," IEEE Transactions on Neural Systems and Rehabilitation Engineering, vol. 22, no. 5, pp. 1041-1052, 2014. 56

[59] G. F. Shannon, "A comparison of alternative means of providing sensory feedback on upper limb prostheses," Medical $\&$ Biological Engineering, no. May, pp. 289-294, 1976. 57

[60] H. Flor, C. Denke, M. Schaefer, and S. Grüsser, "Effect of sensory discrimination training on cortical reorganisation and phantom limb pain," The Lancet, vol. 357, pp. 1763-1764, 2001. 69, 87

[61] M. Botvinick and J. Cohen, "Rubber hands 'feel' touch that eyes see.," Nature, vol. 391, no. 6669, p. 756, 1998. 71, 77, 78, 81

[62] B. Rosén, H. H. Ehrsson, C. Antfolk, C. Cipriani, F. Sebelius, and G. Lundborg, "Referral of sensation to an advanced humanoid robotic hand prosthesis.," Scandinavian journal of plastic and reconstructive surgery and hand surgery / Nordisk plastikkirurgisk forening [and] Nordisk klubb for handkirurgi, vol. 43, no. 5, pp. 260-266, 2009. 72, 81

[63] H. de Preester and M. Tsakiris, "Body-extension versus body-incorporation: Is there a need for a body-model?," Phenomenology and the Cognitive Sciences, vol. 8, no. 3, pp. 307-319, 2009. 72 
[64] M. D'Alonzo and C. Cipriani, "Vibrotactile Sensory Substitution Elicits Feeling of Ownership of an Alien Hand," PLoS ONE, vol. 7, no. 11, 2012. 72, 81

[65] M. R. Mulvey, H. J. Fawkner, H. E. Radford, and M. I. Johnson, "Perceptual embodiment of prosthetic limbs by transcutaneous electrical nerve stimulation," Neuromodulation, vol. 15, no. 1, pp. 42-47, 2012. 72

[66] H. H. Ehrsson, B. Rosén, A. Stockselius, C. Ragnö, P. Köhler, and G. Lundborg, "Upper limb amputees can be induced to experience a rubber hand as their own," Brain, vol. 131, no. 12, pp. 3443-3452, 2008. 72, 81

[67] S. Shimada, K. Fukuda, and K. Hiraki, "Rubber hand illusion under delayed visual feedback," PLoS ONE, vol. 4, no. 7, pp. 1-5, 2009. 78

[68] M. A. F. Ismail and S. Shimada, "Robot' Hand Illusion under Delayed Visual Feedback: Relationship between the Senses of Ownership and Agency," Plos One, vol. 11, no. 7, p. e0159619, 2016. 78

[69] G. L. Moseley, N. Olthof, A. Venema, S. Don, M. Wijers, A. Gallace, and C. Spence, "Psychologically induced cooling of a specific body part caused by the illusory ownership of an artificial counterpart.," Proceedings of the National Academy of Sciences of the United States of America, vol. 105, no. 35 , pp. 13169-13173, 2008. 78,83

[70] M. Tsakiris and P. Haggard, "The rubber hand illusion revisited: visuotactile integration and self-attribution.," Journal of Experimental Psychology: Human Perception and Performance, vol. 31, no. 1, pp. 80-91, 2005. 81, 88

[71] H. Flor, L. Nikolajsen, and T. Staehelin Jensen, "Phantom limb pain: a case of maladaptive CNS plasticity?," Nature Reviews Neuroscience, vol. 7, pp. 873-881, nov 2006. 87 


\section{Appendix A}

\section{Modular test environment for}

\section{closed-loop prosthesis control}

This appendix describes a modular test environment for closed-loop prosthesis control, which was used as a basis for all of the studies presented in this dissertation. It facilitates development and comparison of feedback approaches, control strategies, prosthetic components or feedback interfaces under controlled and repeatable conditions.

The test environment was introduced to the public in a research paper entitled Sensory Feedback in Prosthetics: A Standardized Test Bench for ClosedLoop Control by S. Dosen, M. Markovic, C. Hartmann, and D. Farina (C) 2014 IEEE [29], which was published in the IEEE Transactions on Neural Systems and Rehabilitation Engineering. The following description is based on material from that publication. Since the publication of the paper, the test environment has been further improved in the sense that its components were converted into a standalone library. This allows for even more flexible configuration of setups. The test environment was implemented using Simulink (MathWorks, USA). In order to ensure real-time execution of the test model, the test environment utilises the the Simulink Realtime Windows Target, which bypasses the operating system of the PC and directly allocates resources to the programme.

The two main pillars of the test environment are a component library and the base model for a closed-loop control system.

The component library is comprised of several sub-libraries representing different components of relevance in closed-loop prosthesis control. The basic idea is to connect these components to form a system. The components and their role in a 


\section{A. MODULAR TEST ENVIRONMENT FOR CLOSED-LOOP PROSTHESIS CONTROL}

closed loop system are depicted in Fig. A.1.

Each of the depicted components is represented by a so-called block in the Simulink model. Between these blocks, data flows as multidimensional signals, where all the signals are normalized to intervals $[0,1]$ or $[-1,1]$. Thus it is ensured that all signals can be processed generically, and each implementation of a component can expect its input signals to adhere to these well-defined ranges. As a result, the components of this test environment can be customized into a number of specific configurations by selecting a particular implementation for each of the generic blocks. For each component of the system, a library of blocks to choose from was implemented.

The input interface library implements components for the generation, acquisition and preprocessing of signals from different information sources (e.g., muscle activity, joystick, data file). The output of this component can either be data supplied in real time by the user (e.g., when using a joystick or myoelectric control), real-time sensor data, or predefined signals read from a file implementing specific test scenarios. Relevant implementations for the work presented in this dissertation are blocks for acquisition of multichannel surface EMG, single axis analogue joystick data, and sensor data from an artificial sensor finger (BioTac, Syntouch LLC, USA).

Input blocks conveniently feature a range of optional choices for signal preprocessing, such as baseline correction, filtering, noise suppression or root mean square calculation.

The control approach library provides blocks implementing different control strategies for prosthesis systems. The purpose of the control approach block is to process the input data according to a control strategy, and generate bipolar signals. Those bipolar output signals correspond to the degrees of freedom of the controlled system, for instance, in a single degree-of-freedom prosthesis with hand opening and closing, a single bipolar control signal drives both closing and opening, respectively.

The controlled system component encapsulates a low-level interface to a real or simulated system that is controlled in the closed loop. The block implementations include drivers for hardware interfacing, as well as options for mapping the input control signals to available system degrees of freedom. The output signals from this type of block are in the range of $[0,1]$ and represent sensor data assessing the current state of the system, such as joint positions or exerted interaction force. The components relevant for this work are a block simulating an inverted pendulum, and a block interfacing a commercial hand prosthesis (Michelangelo hand, Otto Bock HealthCare GmbH, Germany).

The experimental task library provides optional components which allow easy implementation and configuration of experimental protocols. Implementations of this component generally provide target signals representing the goal of an 


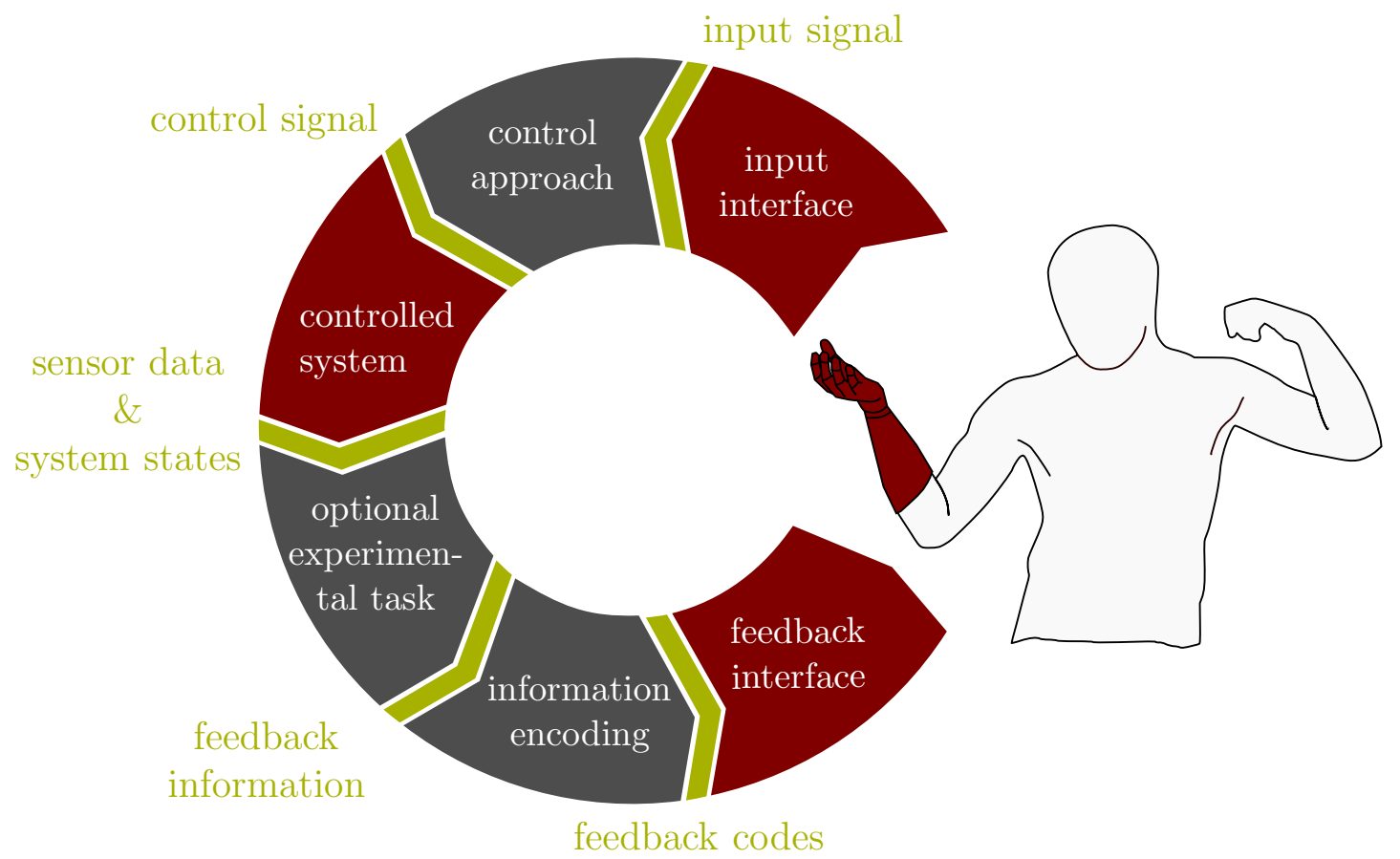

\section{data flow within the model}

purely virtual component, e.g. pattern recognition for prosthesis control

component with potential interface to physical system, e.g. data acquisition card or prosthesis

Figure A.1: Basic components of the modular test environment for closed-loop prosthesis control. The input interface incorporates acquisition of physical data and provides them as input data to the component implementing the prosthesis control approach. The system interface relays the control signals output by the control component to the prosthesis, and acquires sensor data and system states provided by the prosthesis. An optional task component can be utilised to implement experimental protocols. Feedback information (from prosthesis or experimental task) is subsequently encoded into stimulation patterns, which are presented to the user on stimulation devices. These can be interfaced via the stimulation interface component. 


\section{A. MODULAR TEST ENVIRONMENT FOR CLOSED-LOOP PROSTHESIS CONTROL}

experiment, and compare them to the current block input (system states and/or sensor data provided by controlled system). The blocks output the deviation from the target, i.e., the error signal, or a feedback variable based on that error signal. Examples for experimental tasks are compensatory and pursuit tracking with predefined reference trajectories, or "reach the target window" protocols. When the output signals of the controlled system shall be directly used as feedback variables, this optional component can simply be omitted when setting up a model in the test environment.

The information encoding component translates the normalized feedback information signals into the normalized stimulation parameters (i.e., frequency and intensity) for a generic multichannel feedback device. Different generic information encoding schemes can be defined and saved, e.g., single and multichannel spatial encoding and intensity modulations. Each of the input signals, which represent the feedback information, is assigned to the desired mapping, facilitating the implementation of arbitrary feedback configurations. As an example, prosthesis aperture could be chosen to be spatially encoded using an array of electrodes, while at the same time grasping force could be transmitted using a single channel and intensity modulation.

Finally, the feedback interface library encapsulates low-level communication with the device which is used to deliver the feedback. The block implementations for this component include hardware drivers, which handle communication with the feedback device and adjust the stimulation settings according to the normalised feedback codes supplied by the feedback encoding component. Another important feature of this component is that it transforms the normalised feedback codes back into physically sensible stimulation parameters like frequency or current. To this end, user specific ranges for each physical variable are entered and mapped to the normalised ranges. For instance, in the case of electrocutaneous stimulation and intensity encoding by pulse width modulation, it makes sense to map the normalised feedback code domain to the interval delimited by the sensation and comfort threshold pulse widths of the current user. Supported devices include several multichannel electrical stimulators and a tactor controller. Apart from tactile stimulation devices, also several forms of visual feedback components are implemented in this library, e.g., signal scope, object tracking and virtual hand. In general, implementation of new components comprises Simulink implementation of the configurable block, as well as implementation of drivers for communication with hardware devices such as prosthetic components or stimulators. ANSI C and C\# were used for programming drivers, where applicable.

As mentioned before, the second main pillar of the test environment, next to the component library, is the base model. The base model contains an exemplary configuration of the system, including one block from each of the sub-libraries. It further features tools to configure data logging and sampling time options as 
well as a push button to conveniently run the model.

The basic test environment model covers many cases. However, it is of course possible to omit components if not needed for a specific experimental scenario, or to add additional components from the provided libraries, or by directly implementing custom solutions within the Simulink model.

Importantly, since the blocks share a common interface (i.e., normalized, welldefined signals), all the components from the libraries are interoperable and can be therefore combined with virtually no constraints. 